\title{
On periodic solutions for one-phase and two-phase problems of the Navier-Stokes equations
}

\author{
Thomas EITER, Mads Kyed And Yoshiniro Shibata
}

\begin{abstract}
This paper is devoted to proving the existence of time-periodic solutions of one-phase or twophase problems for the Navier-Stokes equations with small periodic external forces when the reference domain is close to a ball. Since our problems are formulated in time-dependent unknown domains, the problems are reduced to quasilinear systems of parabolic equations with non-homogeneous boundary conditions or transmission conditions in fixed domains by using the so-called Hanzawa transform. We separate solutions into the stationary part and the oscillatory part. The linearized equations for the stationary part have eigen-value 0 , which is avoided by changing the equations with the help of the necessary conditions for the existence of solutions to the original problems. To treat the oscillatory part, we establish the maximal $L_{p}-L_{q}$ regularity theorem of the periodic solutions for the system of parabolic equations with non-homogeneous boundary conditions or transmission conditions, which is obtained by the systematic use of $\mathcal{R}$-solvers developed in Shibata (Diff Int Eqns 27(3-4):313-368, 2014; On the $\mathcal{R}$-bounded solution operators in the study of free boundary problem for the Navier-Stokes equations. In: Shibata Y, Suzuki Y (eds) Springer proceedings in mathematics \& statistics, vol. 183, Mathematical Fluid Dynamics, Present and Future, Tokyo, Japan, November 2014, pp 203-285, 2016; Comm Pure Appl Anal 17(4): 1681-1721. https://doi.org/10.3934/cpaa.2018081, 2018; $\mathcal{R}$ boundedness, maximal regularity and free boundary problems for the Navier Stokes equations, Preprint 1905.12900v1 [math.AP] 30 May 2019) to the resolvent problem for the linearized equations and the transference theorem obtained in Eiter et al. ( $\mathcal{R}$-solvers and their application to periodic $L_{p}$ estimates, Preprint in 2019) for the $L_{p}$ boundedness of operator-valued Fourier multipliers. These approaches are the novelty of this paper.
\end{abstract}

\section{Introduction}

This paper is concerned with time-periodic solutions of one-phase and two-phase problems for the Navier-Stokes equations. The periodic solutions for the NavierStokes equations have been studied in many articles [3-8, 10-14,20,23] and references therein. One well-known approach to prove the existence of periodic solutions is the utilization of the Poincaré operator, which maps an initial value into the solution of the PDE at time $\mathcal{T}$, where $\mathcal{T}$ is the period of the data. A fixed point of the Poincaré operator yields an initial value that induces a $\mathcal{T}$-time-periodic solution. Such a utilization of the Poincaré operator is naturally carried out under the global well-posedness of the corresponding initial-boundary value problem for the bounded data on the right hand

Mads Kyed: Joint Appointment Professor of Waseda University. Y. Shibata: Partially supported by JSPS Grant-in-aid for Scientific Research (A) 17H0109, and Top Global University Project. Adjunct Faculty member in the Department of Mechanical Engineering and Materials Science, University of Pittsburgh. 
side of the equations. In the bounded domain case, this is deeply related with the situation where 0 does not belong to the spectrum of the system of the linearized equations. However, in many interesting problems in mathematical physics, we meet the situation that 0 is in the spectrum. One-phase or two-phase problems for the NavierStokes equations are typical examples. As explained in Sects. 1 and 2, the one-phase and two-phase problems we treat in this paper are formulated by the Navier-Stokes equations with free boundary condition or transmission condition on the interface in a time-dependent domain $\Omega_{t}$, which is also unknown. Usually, $\Omega_{t}$ is transformed to a fixed domain $\Omega$ by introducing an unknown function representing the boundary or the interface of $\Omega_{t}$. Thus, the problem treated here becomes a quasilinear system of equations with nonlinear boundary or transmission conditions. The first of our key approaches is to separate solutions into stationary part and oscillatory part. Then, the zero eigen-value of the linearized equations appears only in the equations for the stationary problem. We change the linearized equations by using some necessary conditions for the unique existence of solutions to avoid eigen-value 0 for the linearized problem. This technique is possible under the separation of the stationary part and the oscillatory part, which does not appear when working with the Poincaré operator. The second is to introduce a systematic approach to the maximal $L_{p}-L_{q}$ regularity for the oscillatory part based solely on the $\mathcal{R}$-solver for the resolvent problem of the linearized equations developed in [15-19] and a transference theorem for the $L_{p}$ boundedness of the operator-valued Fourier multiplier due to Eiter, Kyed and Shibata in [2]. The $L_{p}-$ $L_{q}$ maximal regularity for the oscillatory part of solutions is necessary because our problem is a quasilinear system with non-homogeneous boundary conditions. Since the maximal regularity for the oscillatory part of the periodic solutions does not seem to be well-studied, our systematic approach gives a quite important contribution to the study of systems of parabolic equations with non-homogeneous boundary conditions, which is the novelty of this paper.

\subsection{One-phase problem}

Let $\Omega_{t}$ be a time-dependent domain in the $N$-dimensional Euclidean space $\mathbb{R}^{N}$ $(N \geq 2)$. Let $\Gamma_{t}$ be the boundary of $\Omega_{t}$ and $\mathbf{n}_{t}$ the unit outer normal to $\Gamma_{t}$. We assume that $\Omega_{t}$ is occupied by some incompressible viscous fluid of unit mass density whose viscosity coefficient is a positive constant $\mu$. Let $\mathbf{u}={ }^{\top}\left(u_{1}(x, t), \ldots, u_{N}(x, t)\right)$, $x=\left(x_{1}, \ldots, x_{N}\right) \in \Omega_{t}$, and $\mathfrak{p}=\mathfrak{p}(x, t)$ be the velocity field and the pressure field in $\Omega_{t}$, respectively, where ${ }^{\top} M$ denotes the transposed of $M$. We consider the NavierStokes equations in $\Omega_{t}$ with free boundary condition as follows:

$$
\left\{\begin{array}{cc}
\partial_{t} \mathbf{u}+\mathbf{u} \cdot \nabla \mathbf{u}-\operatorname{Div}(\mu \mathbf{D}(\mathbf{u})-\mathfrak{p I})=\mathbf{f} & \text { in } \Omega_{t}, \\
\operatorname{div} \mathbf{u}=0 & \text { in } \Omega_{t}, \\
(\mu \mathbf{D}(\mathbf{u})-\mathfrak{p} \mathbf{I}) \mathbf{n}_{t}=\sigma H\left(\Gamma_{t}\right) \mathbf{n}_{t} & \text { on } \Gamma_{t}, \\
V_{\Gamma_{t}}=\mathbf{u} \cdot \mathbf{n}_{t} & \text { on } \Gamma_{t}
\end{array}\right.
$$


for $t \in \mathbb{R}$. Here, $\mathbf{f}=\mathbf{f}(x, t)$ is a prescribed time-periodic external force with period $2 \pi$; $H\left(\Gamma_{t}\right)$ denotes the $(N-1)$-fold mean curvature of $\Gamma_{t}$ which is given by $H\left(\Gamma_{t}\right) \mathbf{n}_{t}=$ $\Delta_{\Gamma_{t}} x$ for $x \in \Gamma_{t}$, where $\Delta_{\Gamma_{t}}$ is the Laplace-Beltrami operator on $\Gamma_{t} ; V_{\Gamma_{t}}$ is the evolution speed of $\Gamma_{t}$ along $\mathbf{n}_{t} ; \sigma$ is a positive constant representing the surface tension coefficient; $\mathbf{D}(\mathbf{u})$ is the doubled deformation tensor given by $\mathbf{D}(\mathbf{u})=\nabla \mathbf{u}+{ }^{\top} \nabla \mathbf{u}$; and $\mathbf{I}$ is the $(N \times N)$-identity matrix. Moreover, for any $(N \times N)$-matrix of functions $\mathbf{K}$ whose $(i, j)$ th component is $K_{i j}$, Div $K$ is an $N$-vector whose $i$ th component is $\sum_{j=1}^{n} \partial_{j} K_{i j}$ and for any $N$-vector of functions $\mathbf{v}={ }^{\top}\left(v_{1}, \ldots, v_{N}\right), \mathbf{v} \cdot \nabla \mathbf{v}$ is an $N$-vector of functions whose $i$ th component is $\sum_{j=1}^{N} v_{j} \partial_{j} v_{i}$, where $\partial_{j}=\partial / \partial x_{j}$.

Our problem is to find $\Omega_{t}, \Gamma_{t}$, $\mathbf{u}$ and $\mathfrak{p}$ satisfying the periodic condition:

$$
\Omega_{t}=\Omega_{t+2 \pi}, \quad \Gamma_{t}=\Gamma_{t+2 \pi}, \quad \mathbf{u}(x, t)=\mathbf{u}(x, t+2 \pi), \quad \mathfrak{p}(x, t)=\mathfrak{p}(x, t+2 \pi)
$$

for any $t \in \mathbb{R}$.

To state the main result, we introduce assumptions and some functional spaces. Let $\mathbf{p}_{i}=\mathbf{e}_{i}={ }^{T}(0, \ldots, 0, \stackrel{i}{i-t h}, 0, \ldots, 0)$ for $i=1, \ldots, N$ and $\mathbf{p}_{\ell}(\ell=N+1, \ldots, M)$ be one of $x_{i} \mathbf{e}_{j}-x_{j} \mathbf{e}_{i}(1 \leq i<j \leq N)$. Notice that $\mathbf{p}_{\ell}$ forms a basis of the rigid space $\{\mathbf{v} \mid \mathbf{D}(\mathbf{v})=0\}$ and the number $M$ is its dimension. We will construct $\Omega_{t}$ satisfying the following two conditions:

$$
\begin{aligned}
& \operatorname{det}\left(\int_{0}^{2 \pi}\left(\mathbf{p}_{\ell}, \mathbf{p}_{m}\right)_{\Omega_{t}} \mathrm{~d} t\right)_{\ell, m=1, \ldots, M} \neq 0, \\
& \int_{0}^{2 \pi}\left(\int_{\Omega_{t}} x \mathrm{~d} x\right) \mathrm{d} t=0, \\
& \left|\Omega_{t}\right|=\left|B_{R}\right| \text { for any } t \in(0,2 \pi) .
\end{aligned}
$$

Here and in the following, $\left(M_{\ell, m}\right)_{\ell, m=1, \ldots, N}$ denotes an $(N \times N)$-matrix whose $(\ell, m)$ th component is $M_{\ell, m}$; for any domain $G$ and $(N-1)$-dimensional hypersurface $S$, we let

$$
(f, g)_{G}=\int_{G} f(x) \cdot \overline{g(x)} \mathrm{d} x, \quad(f, g)_{S}=\int_{S} f(x) \cdot \overline{g(x)} \mathrm{d} \sigma,
$$

where $\overline{g(x)}$ denotes the complex conjugate of $g(x)$, and $\mathrm{d} \sigma$ the surface element of $S$. $|G|$ denotes the Lebesgue measure of a Lebesgue measurable set $G$ of $\mathbb{R}^{N}$; and $B_{R}$ is the ball with radius $R$ centered at the origin. For $1<p<\infty$ and any Banach space $X$ with norm $\|\cdot\|_{X}$, let

$$
\begin{aligned}
L_{p, \text { per }}((0,2 \pi), X) & =\left\{f: \mathbb{R} \rightarrow X \mid\|f(\cdot)\|_{X} \in L_{1, \text { loc }}(\mathbb{R}),\right. \\
f(t+2 \pi) & =f(t) \text { for any } t \in \mathbb{R}, \\
\|f\|_{L_{p}((0,2 \pi), X)} & \left.=\left(\int_{0}^{2 \pi}\|f(t)\|_{X}^{p} d t\right)^{1 / p}<\infty\right\}, \\
\left.H_{p, \text { per }}^{1}(0,2 \pi), X\right) & =\left\{f: \mathbb{R} \rightarrow X \mid\|f(t)\|_{X} \in L_{1, \text { loc }}(\mathbb{R}) \text { and }\|\dot{f}(t)\|_{X} \in L_{1, \text { loc }}(\mathbb{R}),\right.
\end{aligned}
$$




$$
\begin{aligned}
f(t) & =f(t+2 \pi), \quad \dot{f}(t)=\dot{f}(t+2 \pi) \quad \text { for any } t \in \mathbb{R}, \\
\|f\|_{H_{p}^{1}((0,2 \pi), X)} & \left.=\left(\int_{0}^{2 \pi}\left(\|f(t)\|_{X}^{p}+\|\dot{f}(t)\|_{X}^{p}\right) \mathrm{d} t\right)^{1 / p}<\infty\right\},
\end{aligned}
$$

where $\dot{f}$ denotes the derivative of $f$ with respect to $t$. Let

$$
\begin{aligned}
\|f\|_{L_{p}((0,2 \pi), X)} & =\left(\int_{0}^{2 \pi}\|f(t)\|_{X}^{p} \mathrm{~d} t\right)^{1 / p}, \\
\|f\|_{H_{p}^{1}((0,2 \pi), X)} & =\|f\|_{L_{p}((0,2 \pi), X)}+\|\dot{f}\|_{L_{p}((0,2 \pi), X)} .
\end{aligned}
$$

For any domain $G$ in $\mathbb{R}^{N}$ and $1 \leq q \leq \infty, L_{q}(G), H_{q}^{m}(G)$, and $B_{q, p}^{s}(G)$ denote the standard Lebesgue, Sobolev, and Besov spaces on $G$, and $\|\cdot\|_{L_{q}(G)},\|\cdot\|_{H_{q}^{m}(G)}$, and $\|\cdot\|_{B_{q, p}^{s}(G)}$ denote their respective norms. For any integer $d, X^{d}$ denotes the $d$-fold product of the space $X$, that is $X^{d}=\left\{\mathbf{g}={ }^{\top}\left(g_{1}, \ldots, g_{d}\right) \mid g_{j} \in X(j=1, \ldots, d)\right\}$, while the norm of $X^{d}$ is denoted by $\|\cdot\|_{X}$ instead of $\|\cdot\|_{X^{d}}$ for simplicity.

The following theorem is our main result concerning time-periodic solutions of the one-phase problem for the Navier-Stokes equations.

Theorem 1. Let $1<p, q<\infty$ and $2 / p+N / q<1$. Let $D \subset B_{R}$ be a domain. Then, there exists a positive constant $\epsilon$ and an injective map $x=\Phi(y, t): B_{R} \rightarrow \mathbb{R}^{N}$ for each $t \in(0,2 \pi)$ with

$$
\Phi \in L_{p, \text { per }}\left((0,2 \pi), H_{q}^{3}\left(B_{R}\right)^{N}\right) \cap H_{p, \text { per }}^{1}\left((0,2 \pi), H_{q}^{2}\left(B_{R}\right)\right)
$$

for which the following assertion holds: If $\mathbf{f} \in L_{p, \mathrm{per}}\left((0,2 \pi), L_{q}(D)^{N}\right)$ satisfies the support condition: $\operatorname{supp} \mathbf{f}(\cdot, t) \subset D$ for any $t \in(0,2 \pi)$, the orthogonal condition

$$
\int_{0}^{2 \pi}\left(\mathbf{f}(\cdot, t), \mathbf{p}_{\ell}\right)_{D} \mathrm{~d} t=0 \text { for } \ell=1, \ldots, M,
$$

and the smallness condition: $\|\mathbf{f}\|_{L_{p}\left((0,2 \pi), L_{q}(D)^{N}\right)} \leq \epsilon$, then there exist $\mathbf{v}(y, t), \mathfrak{q}(y, t)$, and $\rho(y, t)$ with

$$
\begin{aligned}
& \mathbf{v} \in L_{p, \operatorname{per}}\left((0,2 \pi), H_{q}^{2}\left(B_{R}\right)^{N}\right) \cap H_{p, \text { per }}^{1}\left((0,2 \pi), L_{q}\left(B_{R}\right)^{N}\right), \\
& \mathfrak{q} \in L_{p, \operatorname{per}}\left((0,2 \pi), H_{q}^{1}\left(B_{R}\right)\right), \\
& \rho \in L_{p, \operatorname{per}}\left((0,2 \pi), W_{q}^{3-1 / q}\left(B_{R}\right)^{N}\right) \cap H_{p, p e r}^{1}\left((0,2 \pi), W_{q}^{2-1 / q}\left(S_{R}\right)\right),
\end{aligned}
$$

such that

$$
\begin{aligned}
\Omega_{t}=\{x & \left.=\Phi(y, t) \mid y \in B_{R}\right\}, \quad \mathbf{u}(x, t)=\mathbf{v}\left(\Phi^{-1}(x, t), t\right), \\
\mathfrak{p}(x, t) & =\mathfrak{q}\left(\Phi^{-1}(x, t), t\right),
\end{aligned}
$$

where $\Phi^{-1}(x, t)$ is the inverse map of the correspondence: $x=\Phi(y, t)$ for any $t \in(0,2 \pi)$, are solutions of equations (1.2) satisfying the periodicity condition (1.2), and $\Gamma_{t}$ is given by

$$
\Gamma_{t}=\left\{x=y+R^{-1} \rho(y, t) y+\xi(t) \mid y \in S_{R}\right\}
$$


where $\xi(t)$ is the barycenter point of $\Omega_{t}$ defined by setting

$$
\xi(t)=\frac{1}{\left|\Omega_{t}\right|} \int_{\Omega_{t}} x \mathrm{~d} x .
$$

Moreover, $\mathbf{v}$ and $\rho$ satisfy the estimate:

$$
\begin{aligned}
& \|\mathbf{v}\|_{L_{p}\left((0,2 \pi), H_{q}^{2}\left(B_{R}\right)\right)}+\left\|\partial_{t} \mathbf{v}\right\|_{L_{p}\left((0,2 \pi), L_{q}\left(B_{R}\right)\right)} \\
& \quad+\|\rho\|_{L_{p}\left((0,2 \pi), W_{q}^{3-1 / q}\left(S_{R}\right)\right)}+\left\|\partial_{t} \rho\right\|_{L_{p}\left((0,2 \pi), W_{q}^{2-1 / q}\left(S_{R}\right)\right)} \\
& \quad+\left\|\partial_{t} \rho\right\|_{L_{\infty}\left((0,2 \pi), W_{q}^{1-1 / q}\left(S_{R}\right)\right)} \leq C \epsilon
\end{aligned}
$$

for some constant $C$ independent of $\epsilon$.

Remark 2. In the construction of the map $\Phi$, we see that $\Phi(y, t)=y+R^{-1} \rho(y, t)+$ $\xi(t)$ for $y \in S_{R}$.

\subsection{Two-phase problem}

Let $\Omega_{+t}$ be a time-dependent domain in the $N$-dimensional Euclidean space $\mathbb{R}^{N}$. Let $\Gamma_{t}$ be the boundary of $\Gamma_{t}$ and $\mathbf{n}_{t}$ its unit outer normal. Let $\Omega$ be a bounded domain in $\mathbb{R}^{N}$ and $S$ the boundary of $\Omega$. We assume that $\Omega_{+t} \subset \Omega$ and $\Gamma_{t} \cap S=\emptyset$. Let $\Omega_{-t}=\Omega \backslash\left(\Omega_{+t} \cup \Gamma_{t}\right)$ and set $\Omega_{t}=\Omega_{+t} \cup \Omega_{-t}$. We assume that $\Omega_{ \pm t}$ be occupied by some incompressible viscous fluids of unit mass densities whose viscosity coefficients are positive constants $\mu_{ \pm}$. Let $\mathbf{u}={ }^{\top}\left(u_{1}, \ldots, u_{N}\right)$ and $\mathfrak{p}$ be the velocity field and the pressure field on $\Omega_{t}$, respectively. We consider the following Navier-Stokes equations with transmission condition on $\Gamma_{t}$ and no-slip condition on $S$ :

$$
\left\{\begin{array}{cl}
\partial_{t} \mathbf{u}_{ \pm}+\mathbf{u} \cdot \nabla \mathbf{u}_{ \pm}-\operatorname{Div}\left(\mu \mathbf{D}\left(\mathbf{u}_{ \pm}\right)-\mathfrak{p}_{ \pm} \mathbf{I}\right)=\mathbf{f} & \text { in } \Omega_{ \pm t}, \\
\operatorname{div} \mathbf{u}_{ \pm}=0 & \text { in } \Omega_{ \pm t}, \\
{[[\mu \mathbf{D}(\mathbf{u})-\mathfrak{p} \mathbf{I}]] \mathbf{n}_{t}=\sigma H\left(\Gamma_{t}\right) \mathbf{n}_{t}, \quad[[\mathbf{u}]]=0} & \text { on } \Gamma_{t}, \\
V_{\Gamma_{t}}=\mathbf{u}_{+} \cdot \mathbf{n}_{t} & \text { on } \Gamma_{t}, \\
\mathbf{u}_{-}=0 & \text { on } S
\end{array}\right.
$$

for $t \in \mathbb{R}$, where $\mathbf{f}=\mathbf{f}(x, t)$ is a prescribed time-periodic external force with period $2 \pi ; \mu$ is the viscosity coefficient given by

$$
\mu= \begin{cases}\mu_{+} & \text {in } \Omega_{+t}, \\ \mu_{-} & \text {in } \Omega_{-t} ;\end{cases}
$$

and $[[f]]$ denotes the jump of $f_{ \pm}$defined on $\Omega_{ \pm}$along $\mathbf{n}_{t}$ defined by setting

$$
[[f]]\left(x_{0}\right)=\lim _{\substack{x \rightarrow x_{0} \\ x \in \Omega_{+t}}} f_{+}(x)-\lim _{\substack{x \rightarrow x_{0} \\ x \in \Omega_{-t}}} f_{-}(x) \text { for } x_{0} \in \Gamma_{t} .
$$

The purpose of this paper is also to find $\Omega_{ \pm t}, \Gamma_{t}, \mathbf{u}_{ \pm}$and $\mathfrak{p}_{ \pm}$which satisfy the periodicity condition:

$\Omega_{ \pm t}=\Omega_{ \pm t+2 \pi}, \quad \Gamma_{t}=\Gamma_{t+2 \pi}, \quad \mathbf{u}_{ \pm}(x, t)=\mathbf{u}_{ \pm}(x, t+2 \pi), \mathfrak{p}_{ \pm}(x, t)=\mathfrak{p}_{ \pm}(x, t+2 \pi)$. 
To state a main result, we introduce the assumptions about $\Omega_{t}$ as follows. We assume that $\Omega \supset B_{R}$ for some $R>0$, and that

$$
\begin{aligned}
& \int_{0}^{2 \pi}\left(\int_{\Omega_{+t}} x \mathrm{~d} x\right) \mathrm{d} t=0 \\
& \left|\Omega_{+t}\right|=\left|B_{R}\right| \text { for any } t \in(0,2 \pi) .
\end{aligned}
$$

The following theorem is our main result concerning time-periodic solutions of the two-phase problem for the Navier-Stokes equations.

Theorem 3. Let $1<p, q<\infty$ and $2 / p+N / q<1 . \Omega_{+}=B_{R}$ and $\Omega_{-}=\Omega \backslash\left(B_{R} \cup\right.$ $\left.S_{R}\right)$. Then, there exist a positive constant $\epsilon$ and a bijective map $x=\Phi(y, t)$ from $\Omega$ onto itself such that for any $\mathbf{f} \in L_{p, p e r}\left((0,2 \pi), L_{q}(\Omega)^{N}\right)$ satisfying the smallness condition: $\|\mathbf{f}\|_{L_{p}\left((0,2 \pi), L_{q}(\Omega)\right)} \leq \epsilon$, there exist $\mathbf{v}_{ \pm}(y, t), \mathfrak{q}_{ \pm}(y, t)$ and $\rho(y, t)$ with

$$
\begin{aligned}
\mathbf{v}_{ \pm} & \in L_{p, \operatorname{per}}\left((0,2 \pi), H_{q}^{2}\left(\Omega_{ \pm}\right)^{N}\right) \cap H_{p, \operatorname{per}}^{1}\left((0,2 \pi), L_{q}\left(\Omega_{ \pm}\right)^{N}\right) \\
\mathfrak{q}_{ \pm} & \in L_{p, \operatorname{per}}\left((0,2 \pi), H_{q}^{1}\left(\Omega_{ \pm}\right)\right), \\
\rho & \in L_{p, \operatorname{per}}\left((0,2 \pi), W_{q}^{3-1 / q}\left(S_{R}\right)\right) \cap H_{p, \operatorname{per}}^{1}\left((0,2 \pi), W_{q}^{2-1 / q}\left(S_{R}\right)\right)
\end{aligned}
$$

such that

$$
\begin{aligned}
\Omega_{ \pm t}=\{x & \left.=\Phi(y, t) \mid y \in \Omega_{ \pm}\right\}, \quad \mathbf{u}_{ \pm}(x, t)=\mathbf{v}_{ \pm}\left(\Phi^{-1}(x, t), t\right), \\
\mathfrak{p}_{ \pm}(x, t) & =\mathfrak{q}_{ \pm}\left(\Phi^{-1}(x, t), t\right),
\end{aligned}
$$

where $y=\Phi^{-1}(x, y)$ is the inverse map of $x=\Phi(y, t)$, are solutions of problem (1.9), and $\Gamma_{t}$ is given by

$$
\Gamma_{t}=\left\{x=y+R^{-1} \rho(y, t)+\xi(t) \mid y \in S_{R}\right\},
$$

where $\xi(t)$ is the barycenter point of $\Omega_{+}$defined by setting

$$
\xi(t)=\frac{1}{\left|\Omega_{+t}\right|} \int_{\Omega_{+t}} x \mathrm{~d} x .
$$

Moreover, $\mathbf{v}_{ \pm}$and $\rho$ satisfy the estimate:

$$
\begin{aligned}
& \sum_{ \pm}\left(\left\|\mathbf{v}_{ \pm}\right\|_{L_{p}\left((0,2 \pi), H_{q}^{2}\left(\Omega_{ \pm}\right)\right)}+\left\|\partial_{t} \mathbf{v}_{ \pm}\right\|_{L_{p}\left((0,2 \pi), L_{q}\left(\Omega_{ \pm}\right)\right)}\right) \\
& \quad+\|\rho\|_{L_{p}\left((0,2 \pi), W_{q}^{3-1 / q}\left(S_{R}\right)\right)}+\left\|\partial_{t} \rho\right\|_{L_{p}\left((0,2 \pi), W_{q}^{2-1 / q}\left(S_{R}\right)\right)} \\
& \quad+\left\|\partial_{t} \rho\right\|_{L_{\infty}\left((0,2 \pi), W_{q}^{1-1 / q}\left(S_{R}\right)\right)} \leq C \epsilon
\end{aligned}
$$

for some constant $C$ independent of $\epsilon$.

Method Since the domain $\Omega_{t}$ is unknown, using the Hanzawa transform, we reduce the equations onto a fixed domain, which results in a system of quasilinear equations. Thus, we cannot use the analytic $C_{0}$-semi-group approach. Our main tool is to use the 
$L_{p}-L_{q}$ maximal regularity for periodic solutions to the linearized equations, which can be obtained by using the $\mathcal{R}$-solver to the generalized resolvent problem and applying the transference theorem $([1,2])$ to the solution formula represented by the $\mathcal{R}$-solver. This is a quite new and more direct approach and a completely different idea than exploiting the Poincaré operator.

Further notation This section is ended by explaining further notation used in this paper. We denote the sets of all complex numbers, real numbers, integers, and natural numbers by $\mathbb{C}, \mathbb{R}, \mathbb{Z}$, and $\mathbb{N}$, respectively. Let $\mathbb{N}_{0}=\mathbb{N} \cup\{0\}$. Let $X$ be a Banach space with norm $\|\cdot\|_{X}$. For any $X$-valued function $f: \mathbb{R} \rightarrow X$ the functions $\mathcal{F}[f]$ and $\mathcal{F}^{-1}[f]$ denote the Fourier transform and the inverse Fourier transform of $f$, respectively, defined by setting

$$
\mathcal{F}[f](\tau)=\frac{1}{2 \pi} \int_{\mathbb{R}} \mathrm{e}^{-i \tau t} f(t) \mathrm{d} t, \quad \mathcal{F}^{-1}[f](t)=\int_{\mathbb{R}} \mathrm{e}^{i t \tau} f(\tau) \mathrm{d} \tau .
$$

Let $g: \mathbb{T} \rightarrow X$ be an $X$-valued function defined on the torus $\mathbb{T}=\mathbb{R} / 2 \pi \mathbb{Z}$. We define the Fourier transform $\mathcal{F}_{\mathbb{T}}$ acting on $g$ by setting

$$
\mathcal{F}_{\mathbb{T}}[g](k)=\frac{1}{2 \pi} \int_{0}^{2 \pi} \mathrm{e}^{-i k t} g(t) \mathrm{d} t,
$$

which is regarded as a correspondence $g \mapsto\left(\mathcal{F}_{\mathbb{T}}[g](k)\right)=\left\{\mathcal{F}_{\mathbb{T}}[g](k) \in X \mid k \in \mathbb{Z}\right\}$. For any sequence $\left(a_{k}\right)=\left\{a_{k} \in X \mid k \in \mathbb{Z}\right\}$, we define the inverse Fourier transform $\mathcal{F}_{\mathbb{T}}^{-1}$ acting on $\left(a_{k}\right)$ by setting

$$
\mathcal{F}_{\mathbb{T}}^{-1}\left[\left(a_{k}\right)\right](t)=\sum_{k \in \mathbb{Z}} \mathrm{e}^{i k t} a_{k} .
$$

For any $X$-valued periodic function $f$ with period $2 \pi$, we set

$$
f_{S}=\frac{1}{2 \pi} \int_{0}^{2 \pi} f(t) \mathrm{d} t, \quad f_{\perp}=f-f_{S} .
$$

The $f_{S}$ and $f_{\perp}$ are called stationary part and oscillatory part of $f$, respectively.

For $1 \leq p \leq \infty, L_{p}(\mathbb{R}, X)$ and $H_{p}^{1}(\mathbb{R}, X)$ denote the standard Lebesgue and Sobolev spaces of $X$-valued functions defined on $\mathbb{R}$, and $\|\cdot\|_{L_{p}(\mathbb{R}, X)},\|\cdot\|_{H_{p}^{1}(\mathbb{R}, X)}$ denote their respective norms. For $\theta \in(0,1), H_{p \text {,per }}^{\theta}((0,2 \pi), X)$ denotes the $X$ valued Bessel potential space of periodic functions defined by

$$
\begin{gathered}
H_{p, \text { per }}^{\theta}((0,2 \pi), X)=\left\{f \in L_{p, \operatorname{per}}((0,2 \pi), X) \mid\|f\|_{H_{p}^{\theta}((0,2 \pi), X)}<\infty\right\}, \\
\|f\|_{H_{p}^{\theta}((0,2 \pi), X)}=\left(\int_{0}^{2 \pi}\left\|\mathcal{F}_{\mathbb{T}}^{-1}\left[\left(1+k^{2}\right)^{\theta / 2} \mathcal{F}_{\mathbb{T}}[f](k)\right](t)\right\|_{X}^{p} \mathrm{~d} t\right)^{1 / p}
\end{gathered}
$$

As usual, we set $L_{p \text {,per }}((0,2 \pi), X)=H_{p, \text { per }}^{0}((0,2 \pi), X)$.

For any multi-index $\alpha=\left(\alpha_{1}, \ldots, \alpha_{N}\right) \in \mathbb{N}_{0}^{N}$ we set $\partial_{x}^{\alpha} h=\partial_{1}^{\alpha_{1}} \cdots \partial_{N}^{\alpha_{N}} h$ with $\partial_{i}=\partial / \partial x_{i}$. For any scalar function $f$, we write

$$
\nabla f=\left(\partial_{1} f, \ldots, \partial_{N} f\right), \quad \bar{\nabla} f=\left(f, \partial_{1} f, \ldots, \partial_{N} f\right),
$$




$$
\nabla^{n} f=\left(\partial_{x}^{\alpha} f|| \alpha \mid=n\right), \quad \bar{\nabla}^{n} f=\left(\partial_{x}^{\alpha} f|| \alpha \mid \leq n\right) \quad(n \geq 2),
$$

where $\partial_{x}^{0} f=f$. For any $m$-vector of functions $\mathbf{f}={ }^{\top}\left(f_{1}, \ldots, f_{m}\right)$, we write

$$
\begin{aligned}
& \nabla \mathbf{f}=\left(\nabla f_{1}, \ldots, \nabla f_{m}\right), \quad \bar{\nabla} \mathbf{f}=\left(\bar{\nabla} f_{1}, \ldots, \bar{\nabla} f_{m}\right), \\
& \nabla^{n} \mathbf{f}=\left(\nabla^{n} f_{1}, \ldots, \nabla^{n} f_{m}\right), \quad \bar{\nabla}^{n} \mathbf{f}=\left(\bar{\nabla}^{n} f_{1}, \ldots, \bar{\nabla}^{n} f_{m}\right) .
\end{aligned}
$$

For any $N$-vector of functions, $\mathbf{u}={ }^{\top}\left(u_{1}, \ldots, u_{N}\right)$, sometimes $\nabla \mathbf{u}$ is regarded as an $(N \times N)$-matrix of functions whose $(i, j)$ th component is $\partial_{j} u_{i}$. For any $m$-vector $V=\left(v_{1}, \ldots, v_{m}\right)$ and $n$-vector $W=\left(w_{1}, \ldots, w_{n}\right), V \otimes W$ denotes an $(m \times n)$ matrix whose $(i, j)$ th component is $V_{i} W_{j}$. For any $(m n \times N)$-matrix $A=\left(A_{i j, k} \mid\right.$ $i=1, \ldots, m, j=1, \ldots, n, k=1, \ldots, N), A V \otimes W$ denotes an $N$-column vector whose $k$ th component is the quantity: $\sum_{j=1}^{m} \sum_{j=1}^{n} A_{i j, k} v_{i} w_{j}$.

Let $\mathbf{a} \cdot \mathbf{b}=<\mathbf{a}, \mathbf{b}>=\sum_{j=1}^{N} a_{j} b_{j}$ for any $N$-vectors $\mathbf{a}=\left(a_{1}, \ldots, a_{N}\right)$ and $\mathbf{b}=\left(b_{1}, \ldots, b_{N}\right)$. For any $N$-vector $\mathbf{a}$, let $\Pi_{0} \mathbf{a}=\mathbf{a}_{\tau}:=\mathbf{a}-<\mathbf{a}, \mathbf{n}>\mathbf{n}$. For any two $(N \times N)$-matrices $\mathbf{A}=\left(A_{i j}\right)$ and $\mathbf{B}=\left(B_{i j}\right)$, the quantity $\mathbf{A}: \mathbf{B}$ is defined by $\mathbf{A}: \mathbf{B}=\sum_{i, j=1}^{N} A_{i j} B_{j i}$. For any domain $G$ with boundary $\partial G$, we set

$$
(\mathbf{u}, \mathbf{v})_{G}=\int_{G} \mathbf{u}(x) \cdot \overline{\mathbf{v}(x)} \mathrm{d} x, \quad(\mathbf{u}, \mathbf{v})_{\partial G}=\int_{\partial G} \mathbf{u} \cdot \overline{\mathbf{v}(x)} \mathrm{d} \sigma,
$$

where $\overline{\mathbf{v}(x)}$ is the complex conjugate of $\mathbf{v}(x)$ and $\mathrm{d} \sigma$ denotes the surface element of $\partial G$. Given $1<q<\infty$, let $q^{\prime}=q /(q-1)$. For $L>0$, let $B_{L}=\left\{x \in \mathbb{R}^{N}|| x \mid<L\right\}$ and $S_{L}=\left\{x \in \mathbb{R}^{N}|| x \mid=L\right\}$.

For two Banach spaces $X$ and $Y, X+Y=\{x+y \mid x \in X, y \in Y\}, \mathcal{L}(X, Y)$ denotes the set of all bounded linear operators from $X$ into $Y$ and $\mathcal{L}(X, X)$ is written simply as $\mathcal{L}(X)$. Moreover, let $\mathcal{R}_{\mathcal{L}(X, Y)}(\{\mathcal{T}(\lambda) \mid \lambda \in I\})$ be the $\mathcal{R}$-bound of the operator family $\{\mathcal{T}(\lambda) \mid \lambda \in I\} \subset \mathcal{L}(X, Y)$ (see also Definition 7). Let

$$
i \mathbb{R}=\{i \lambda \in \mathbb{C} \mid \lambda \in \mathbb{R}\}, \quad i \mathbb{R}_{\lambda_{0}}=\left\{i \lambda \in i \mathbb{R}|| \lambda \mid \geq \lambda_{0}\right\} .
$$

The letter $C$ denotes a generic constant and $C_{a, b, c, \ldots}$ denotes that the constant $C_{a, b, c, \ldots}$ depends on $a, b, c, \ldots$; the value of $C$ and $C_{a, b, c, \ldots}$ may change from line to line.

\section{Linearization principle}

We now formulate the problems (1.1) and (1.9) in a fixed domain and state main results in this setting. Theorems 1 and 3 follow from the main theorems of this section.

2.1. One-phase problem

Let $\Omega_{t}, \mathbf{u}$ and $\mathfrak{p}$ satisfies equations (1.1) and the periodicity condition (1.2). We have

$$
\left((\mu \mathbf{D}(\mathbf{u})-\mathfrak{p I}) \mathbf{n}_{t}, \mathbf{e}_{i}\right)_{\Gamma_{t}}=\sigma\left(\Delta_{\Gamma_{t}} x, \mathbf{e}_{i}\right)_{\Gamma_{t}}=-\sigma\left(\nabla_{\Gamma_{t}} x, \nabla_{\Gamma_{t}} \mathbf{e}_{i}\right)_{\Gamma_{t}}=0
$$




$$
\begin{aligned}
& \left((\mu \mathbf{D}(\mathbf{u})-\mathfrak{p} \mathbf{I}) \mathbf{n}_{t}, x_{i} \mathbf{e}_{j}-x_{j} \mathbf{e}_{i}\right)_{\Gamma_{t}}=\sigma\left(\Delta_{\Gamma_{t}} x, x_{i} \mathbf{e}_{j}-x_{j} \mathbf{e}_{i}\right)_{\Gamma_{t}} \\
& \quad=-\sigma\left(\nabla_{\Gamma_{t}} x_{j}, \nabla_{\Gamma_{t}} x_{i}\right)_{\Gamma_{t}}+\sigma\left(\nabla_{\Gamma_{t}} x_{i}, \nabla_{\Gamma_{t}} x_{j}\right)_{\Gamma_{t}}=0 .
\end{aligned}
$$

Multiplying the first equation in (1.1) with $\mathbf{p}_{\ell}$ and integrating the resultant formula on $\Omega_{t}$ and using the divergence theorem of Gauss give that

$$
\frac{d}{\mathrm{~d} t}\left(\mathbf{u}, \mathbf{p}_{\ell}\right)_{\Omega_{t}}=\left(\mathbf{f}, \mathbf{p}_{\ell}\right)_{\Omega_{t}}
$$

In fact, we have used the fact that

$$
\frac{d}{\mathrm{~d} t} \int_{\Omega_{t}} \mathbf{u}(x, t) \cdot \mathbf{p}_{\ell}(x) \mathrm{d} x=\int_{\Omega_{t}}<\partial_{t} \mathbf{u}+\mathbf{u} \cdot \nabla \mathbf{u}, \mathbf{p}_{\ell}>\mathrm{d} x,
$$

which follows from the Reynolds transport theorem ${ }^{1}$ and that $\operatorname{div} \mathbf{u}=0$ in $\Omega_{t}$. Thus, the periodicity condition (1.2) yields that

$$
\int_{0}^{2 \pi}\left(\int_{D} \mathbf{f}(x, \cdot) \cdot \mathbf{p}_{\ell}(x) \mathrm{d} x\right) \mathrm{d} t=0 \text { for } \ell=1, \ldots, M,
$$

where we have used the assumption that $\operatorname{supp} \mathbf{f}(\cdot, t) \subset D$ for any $t \in \mathbb{R}$. Thus, the condition (1.6) is a necessary one to prove Theorem 1. From this observation, instead of problem (1.2), we consider the following equations:

$$
\begin{cases}\partial_{t} \mathbf{u}+\mathbf{u} \cdot \nabla \mathbf{u}-\operatorname{Div}(\mu \mathbf{D}(\mathbf{u})-\mathfrak{p} \mathbf{I})+\sum_{k=1}^{M} \int_{0}^{2 \pi}\left(\mathbf{u}(\cdot, t), \mathbf{p}_{k}\right) \Omega_{t} \mathrm{~d} t \mathbf{p}_{k}=\mathbf{f} & \text { in } \Omega_{t}, \\ \quad \operatorname{div} \mathbf{u}=0 & \text { in } \Omega_{t}, \\ (\mu \mathbf{D}(\mathbf{u})-\mathfrak{p} \mathbf{I}) \mathbf{n}_{t}=\sigma H\left(\Gamma_{t}\right) \mathbf{n}_{t} & \text { on } \Gamma_{t}, \\ V_{\Gamma_{t}}=\mathbf{u} \cdot \mathbf{n}_{t} & \text { on } \Gamma_{t}\end{cases}
$$

for $t \in \mathbb{R}$. In fact, if $\Omega_{t}, \mathbf{u}$ and $\mathfrak{p}$ satisfy equations (2.2), then we have

$$
\frac{d}{\mathrm{~d} t}\left(\mathbf{u}(\cdot, t), \mathbf{p}_{\ell}\right)_{\Omega_{t}}+\sum_{k=1}^{M} \int_{0}^{2 \pi}\left(\mathbf{u}(\cdot, t), \mathbf{p}_{k}\right)_{\Omega_{t}} \mathrm{~d} t\left(\mathbf{p}_{k}, \mathbf{p}_{\ell}\right)_{\Omega_{t}}=\left(\mathbf{f}, \mathbf{p}_{\ell}\right)_{\Omega_{t}},
$$

which, combined with the periodicity condition (1.2), the assumption (1.3) and (2.1), leads to

$$
\int_{0}^{2 \pi}\left(\mathbf{u}(\cdot, t), \mathbf{p}_{k}\right)_{\Omega_{t}} \mathrm{~d} t=0 \text { for } k=1, \ldots, M
$$

\footnotetext{
${ }^{1}$ For any $f(x, t)$ defined on $\Omega_{t}$, we have

$$
\frac{d}{\mathrm{~d} t} \int_{\Omega_{t}} f(x, t) \mathrm{d} x=\int_{\Omega_{t}}\left(\partial_{t} f+\operatorname{div}(f \mathbf{u})\right) \mathrm{d} x,
$$
}

which is called the Reynolds transport theorem. 
Thus, $\Omega_{t}, \mathbf{u}$ and $\mathfrak{p}$ satisfy the first equation in (1.1). Therefore, under the stated assumptions, a solution to problem (2.2) is a solution to the original problem (1.1). However, as we shall see below, the condition (2.1) is not necessary to find a solution to (2.2).

From now on, we consider problem (2.2). We reduce problem (2.2) to some nonlinear equations on $B_{R}$ by using the Hanzawa transform, which we explain below. Let $\xi(t)$ be the barycenter point of $\Omega_{t}$ defined by setting

$$
\xi(t)=\frac{1}{\left|B_{R}\right|} \int_{\Omega_{t}} x \mathrm{~d} x,
$$

where we have used the fact that $\left|\Omega_{t}\right|=\left|B_{R}\right|$, which follows from the assumption (1.5). By the Reynolds transport theorem, we see that

$$
\frac{d}{\mathrm{~d} t} \xi(t)=\frac{1}{\left|B_{R}\right|} \int_{\Omega_{t}}\left(\partial_{t} x+\mathbf{u} \cdot \nabla x\right) \mathrm{d} x=\frac{1}{\left|B_{R}\right|} \int_{\Omega_{t}} \mathbf{u}(x, t) \mathrm{d} x
$$

because $\operatorname{div} \mathbf{u}=0$. Let $\rho(y, t)$ be an unknown time-periodic function with period $2 \pi$ such that

$$
\Gamma_{t}=\left\{x=y+\rho(y, t) \mathbf{n}+\xi(t) \mid y \in S_{R}\right\},
$$

where $S_{R}=\left\{x \in \mathbb{R}^{N}|| x \mid=R\right\}$ and $\mathbf{n}$ is the unit outer normal to $S_{R}$, that is $\mathbf{n}=x /|x|$ for $x \in S_{R}$. Let $H_{\rho}$ be a suitable extension of $\rho$ to $\mathbb{R}^{N}$, and then by the $K$-method in the theory of real interpolation [9,21], we see that there exist constants $C_{1}$ and $C_{2}$ such that

$$
\begin{aligned}
& C_{1}\left\|H_{\rho}(\cdot, t)\right\|_{H_{q}^{k}\left(\mathbb{R}^{N}\right)} \leq\|\rho(\cdot, t)\|_{W_{q}^{k-1 / q}\left(S_{R}\right)} \leq C_{2}\left\|H_{\rho}(\cdot, t)\right\|_{H_{q}^{k}\left(\mathbb{R}^{N}\right)} \text { for } k=1,2,3, \\
& C_{1}\left\|\partial_{t} H_{\rho}(\cdot, t)\right\|_{H_{q}^{k}\left(\mathbb{R}^{N}\right)} \leq\left\|\partial_{t} \rho(\cdot, t)\right\|_{W_{q}^{k-1 / q}\left(S_{R}\right)} \leq C_{2}\left\|\partial_{t} H_{\rho}(\cdot, t)\right\|_{H_{q}^{k}\left(\mathbb{R}^{N}\right)} \text { for } k=1,2,
\end{aligned}
$$

for any $t \in(0,2 \pi)$. In the following, we fix the method of this extension. For example, $\hat{H}_{\rho}$ is the unique solution of the Dirichlet problem:

$$
(1-\Delta) \hat{H}_{\rho}=0 \text { in } \mathbb{R}^{N} \backslash S_{R},\left.\quad \hat{H}_{\rho}\right|_{S_{R}}=\rho .
$$

Let $\varphi$ be a $C^{\infty}\left(\mathbb{R}^{N}\right)$ function which equals one for $x \in B_{2 R}$ and zero for $x \notin B_{3 R}$, and we set $H_{\rho}=\varphi \hat{H}_{\rho}$. We assume that

$$
\sup _{t \in \mathbb{R}}\left\|\nabla H_{\rho}(\cdot, t)\right\|_{H_{\infty}^{1}\left(\mathbb{R}^{N}\right)} \leq \delta
$$

with some small constant $\delta>0$. Notice that $y /|y|=R^{-1} y$ for $y \in S_{R}$ is the unit outer normal to $S_{R}$. Let $\Phi(y, t)=y+R^{-1} H_{\rho}(y, t) y+\xi(t)$. We choose $\delta>0$ so small that the map $x=\Phi(y, t)$ is injective. In fact, for any $y_{1}$ and $y_{2}$

$$
\begin{aligned}
\left|\Phi\left(y_{1}, t\right)-\Phi\left(y_{2}, t\right)\right| & \geq\left|y_{1}-y_{2}\right|-\sup _{t \in \mathbb{R}}\left\|\nabla H_{\rho}(\cdot, t)\right\|_{H_{\infty}^{1}\left(\mathbb{R}^{N}\right)}\left|y_{1}-y_{2}\right| \\
& \geq(1-\delta)\left|y_{1}-y_{2}\right|,
\end{aligned}
$$


which leads to the injectivity of the transformation $x=\Phi(y, t)$ for any $t \in \mathbb{R}$ provided that $0<\delta<1$. Moreover, using the inverse mapping theorem, we see that the map $x=\Phi(y, t)$ is surjective from $\mathbb{R}^{N}$ onto $\mathbb{R}^{N}$.

Let

$$
\begin{aligned}
& \Omega_{t}=\left\{x=y+R^{-1} H_{\rho}(y, t) y+\xi(t) \mid y \in B_{R}\right\}, \\
& \Gamma_{t}=\left\{x=y+R^{-1} \rho(y, t) y+\xi(t) \mid y \in S_{R}\right\} .
\end{aligned}
$$

Let $\mathbf{u}(x, t)$ and $\mathfrak{p}(x, t)$ satisfy equations (1.1), and let $\mathbf{v}(y, t)=\mathbf{u}(x, t)$ and $\mathfrak{q}(y, t)=$ $\mathfrak{p}(x, t)$. We derive an equation for $\mathbf{v}$ and $\rho$ from the kinematic condition: $V_{\Gamma_{t}}=\mathbf{u} \cdot \mathbf{n}_{t}$ on $\Gamma_{t}$. From the definition:

$$
V_{\Gamma_{t}}=\frac{\partial x}{\partial t} \cdot \mathbf{n}_{t}=\left(\frac{\partial \rho}{\partial t} \mathbf{n}+\xi^{\prime}(t)\right) \cdot \mathbf{n}_{t} .
$$

To represent $\xi^{\prime}(t)$, we introduce the Jacobian $J(t)$ of the transformation $x=\Phi(y, t)$, which is written as $J(t)=1+J_{0}(t)$ with

$$
J_{0}(t)=\operatorname{det}\left(\delta_{i j}+R^{-1} \frac{\partial}{\partial y_{i}}\left(H_{\rho}(y, t) y_{j}\right)\right)_{i, j=1, \ldots, N}-1 .
$$

Choosing $\delta>0$ small enough in (2.6), we have

$$
\left|J_{0}(t)\right| \leq C\left\|\nabla H_{\rho}(\cdot, t)\right\|_{L_{\infty}\left(B_{R}\right)}
$$

From (2.4) it follows that

$$
\xi^{\prime}(t)=\frac{1}{\left|B_{R}\right|} \int_{B_{R}} \mathbf{v}(y, t) \mathrm{d} y+\frac{1}{\left|B_{R}\right|} \int_{B_{R}} \mathbf{v}(y, t) J_{0}(t) \mathrm{d} y,
$$

and so noting that $\mathbf{n} \cdot \mathbf{n}=1$, we have the kinematic equation:

$$
\partial_{t} \rho-\left(\mathbf{v}-\frac{1}{\left|B_{R}\right|} \int_{B_{R}} \mathbf{v}(y, t) \mathrm{d} y\right) \cdot \mathbf{n}=d(\mathbf{v}, \rho)
$$

with

$$
d(\mathbf{v}, \rho)=\frac{1}{\left|B_{R}\right|} \int_{B_{R}} \mathbf{v}(y, t) J_{0}(t) \mathrm{d} y \cdot\left(\mathbf{n}-\mathbf{n}_{t}\right)+\frac{\partial \rho}{\partial t} \mathbf{n} \cdot\left(\mathbf{n}-\mathbf{n}_{t}\right)+\mathbf{v} \cdot\left(\mathbf{n}_{t}-\mathbf{n}\right) .
$$

As will be seen in Sect. 3, we have $<H\left(\Gamma_{t}\right) \mathbf{n}_{t}, \mathbf{n}_{t}>=\left(\Delta_{S_{R}}+(N-1) / R^{2}\right) \rho-(N-$ $1) / R+$ nonlinear terms, and $-(N-1) / R^{2}$ is the first eigen-value of the LaplaceBeltrami operator $\Delta_{S_{R}}$ on $S_{R}$ with eigen-functions $y_{j} / R$ for $y=\left(y_{1}, \ldots, y_{N}\right) \in S_{R}$. We need to derive some auxiliary equations to avoid the zero and first eigen-values of $\Delta_{S_{R}}$. From the assumption (1.5) and the representation formulas of $\Omega_{t}$ and $\Gamma_{t}$ in (2.7), by using polar coordinates we have

$$
\left|B_{R}\right|=\left|\Omega_{t}\right|=\int_{S_{R}}\left(\int_{0}^{1+R^{-1} \rho(\omega, t)} r^{N-1} d r\right) \mathrm{d} \omega=\frac{1}{N} \int_{S_{R}}\left(1+R^{-1} \rho(\omega, t)\right)^{N} \mathrm{~d} \omega
$$




$$
=\left|B_{R}\right|+R^{-1} \int_{S_{R}} \rho \mathrm{d} \omega+\sum_{k=2}^{N} \frac{{ }_{N} C_{k}}{N} R^{-k} \int_{S_{R}} \rho^{k} \mathrm{~d} \omega,
$$

and so we have

$$
\int_{S_{R}} \rho \mathrm{d} \omega+\sum_{k=2}^{N} \frac{{ }_{N} C_{k}}{N} R^{1-k} \int_{S_{R}} \rho^{k} \mathrm{~d} \omega=0
$$

where d $\omega$ denotes the surface element of $S_{R}$. Moreover, from (2.3) and the assumption (1.5), using polar coordinates centered at $\xi(t)$, we have

$$
\begin{aligned}
0 & =\frac{1}{\left|B_{R}\right|} \int_{\Omega_{t}}(x-\xi(t)) \mathrm{d} x=\frac{1}{\left|B_{R}\right|} \int_{S_{R}}\left(\int_{0}^{1+R^{-1} \rho(\omega, t)} r^{N} \omega d r\right) \mathrm{d} \omega \\
& =\frac{1}{\left|B_{R}\right|} \frac{1}{N+1} \int_{S_{R}}\left(1+R^{-1} \rho(\omega, t)\right)^{N+1} \omega \mathrm{d} \omega \\
& =\frac{1}{\left|B_{R}\right|}\left(R^{-1} \int_{S_{R}} \rho \omega \mathrm{d} \omega+\sum_{k=2}^{N+1} \frac{N+1 C_{k}}{N+1} R^{-k} \int_{S_{R}} \rho^{k} \omega \mathrm{d} \omega\right)
\end{aligned}
$$

from which it follows that

$$
\int_{S_{R}} \rho \omega_{j} \mathrm{~d} \omega+\sum_{k=2}^{N+1} \frac{N+1 C_{k}}{N+1} R^{1-k} \int_{S_{R}} \rho^{k} \omega_{j} \mathrm{~d} \omega=0
$$

for $j=1, \ldots, N$. Thus, under the assumption (1.5) and the representation of $\Gamma_{t}$ and $\Omega_{t}$ in (2.7), the kinematic condition (2.10) is equivalent to the equation

$$
\begin{aligned}
& \partial_{t} \rho+\int_{S_{R}} \rho \mathrm{d} \omega+\sum_{k=1}^{N}\left(\int_{S_{R}} \rho \omega_{k} \mathrm{~d} \omega\right) y_{k} \\
& -\left(\mathbf{v}-\frac{1}{\left|B_{R}\right|} \int_{B_{R}} \mathbf{v} \mathrm{d} y\right) \cdot \mathbf{n}=\tilde{d}(\mathbf{v}, \rho) \text { on } S_{R} \times(0,2 \pi)
\end{aligned}
$$

with

$$
\tilde{d}(\mathbf{v}, \rho)=d(\mathbf{v}, \rho)-\sum_{k=2}^{N} \frac{{ }_{N} C_{k}}{N} R^{1-k} \int_{S_{R}} \rho^{k} \mathrm{~d} \omega-\sum_{k=2}^{N+1} \frac{N+1 C_{k}}{N+1} R^{1-k}\left(\int_{S_{R}} \rho^{k} \omega \mathrm{d} \omega\right) y_{k} .
$$

Therefore, to prove the existence of $\left(\Omega_{t}, \mathbf{u}, \mathfrak{p}\right)$, we shall prove the well-posedness of the following equations:

$$
\begin{cases}\partial_{t} \mathbf{v}+\mathcal{L} \mathbf{v}_{S}-\operatorname{Div}(\mu(\mathbf{D}(\mathbf{v})-\mathfrak{q} \mathbf{I})=\mathbf{G}+\mathbf{F}(\mathbf{v}, \rho) & \text { in } B_{R} \times(0,2 \pi) \\ \operatorname{div} \mathbf{v}=g(\mathbf{v}, \rho)=\operatorname{div} \mathbf{g}(\mathbf{v}, \rho) & \text { in } B_{R} \times(0,2 \pi) \\ \partial_{t} \rho+\mathcal{M} \rho-\mathcal{A} \mathbf{v} \cdot \mathbf{n}=\tilde{d}(\mathbf{v}, \rho) & \text { on } S_{R} \times(0,2 \pi) \\ (\mu \mathbf{D}(\mathbf{v})-\mathfrak{q}) \mathbf{n}-\left(\mathcal{B}_{R} \rho\right) \mathbf{n}=\mathbf{h}(\mathbf{v}, \rho) & \text { on } S_{R} \times(0,2 \pi)\end{cases}
$$


where we have set

$$
\begin{aligned}
& \mathcal{L} \mathbf{v}_{S}=\sum_{k=1}^{M}\left(\mathbf{v}_{S}, \mathbf{p}_{k}\right)_{B_{R}} \mathbf{p}_{k} ; \quad \mathcal{A} \mathbf{v}=\mathbf{v}-\frac{1}{\left|B_{R}\right|} \int_{B_{R}} \mathbf{v} \mathrm{d} y ; \\
& \mathcal{M} \rho=\int_{S_{R}} \rho \mathrm{d} \omega+\sum_{k=1}^{N}\left(\int_{S_{R}} \rho \omega_{k} \mathrm{~d} \omega\right) y_{k} ; \\
& \mathcal{B}_{R} \rho=\left(\Delta_{S_{R}}+\frac{N-1}{R^{2}}\right) \rho=R^{-2}\left(\Delta_{S_{1}}+(N-1)\right) \rho
\end{aligned}
$$

where $\Delta_{S_{1}}$ is the Laplace-Beltrami operator on the unit sphere $S_{1}$. For the functions on the right side of equations (2.16), $\mathbf{G}(y, t)$ and $\mathbf{F}(\mathbf{v}, \rho)$ are given in (3.13) in Sect. $3, g(\mathbf{v}, \rho)$ and $\mathbf{g}(\mathbf{v}, \rho)$ given in (3.6) in Sect. 3, $\tilde{d}(\mathbf{v}, \rho)$ has been given in (2.15) and $\mathbf{h}(\mathbf{v}, \rho)=\left(\mathbf{h}^{\prime}(\mathbf{v}, \rho), h_{N}(\mathbf{v}, \rho)\right)$ is given in (3.31) and (3.34) in Sect. 3.

The following theorem is the unique existence theorem of $2 \pi$-periodic solutions of problem (2.16).

Theorem 4. Let $1<p, q<\infty$ and $2 / p+N / q<1$. Then, there exists a small constant $\epsilon>0$ such that if $\mathbf{f}$ satisfies the assumption (1.6) and the smallness condition: $\|\mathbf{f}\|_{L_{p}\left((0,2 \pi), L_{q}(D)\right)} \leq \epsilon$, then problem (2.16) admits $2 \pi$-periodic solutions $\mathbf{v}, \mathfrak{q}$, and $\rho$ satisfying the regularity condition (1.7) and the estimate (1.8) in Theorem 1.

Proof of Theorem 1. We prove Theorem 1 with the help of Theorem 4. Let $\xi(t)$ be defined by

$$
\xi(t)=\int_{0}^{t} \xi^{\prime}(s) \mathrm{d} s+c
$$

where $c$ is chosen in such a way that

$$
\int_{0}^{2 \pi} \xi(s) \mathrm{d} s=0 .
$$

Here, $\xi^{\prime}(t)$ is given by the formula in (2.9). Then, we define $\Omega_{t}$ and $\Gamma_{t}$ by the formulas in (2.7). Let $\Phi(y, t)=y+R^{-1} H_{\rho} y+\xi(t)$. By choosing $\epsilon$ sufficiently small, estimates (1.8) and (2.5) ensure that the condition (2.6) is satisfied with small $\delta>0$. This yields the existence of the inverse map $y=\Phi^{-1}(x, t)$ of the map: $x=\Phi(y, t)$. Thus, the velocity field $\mathbf{u}(x, t)$ and the pressure $\mathfrak{p}(x, t)$ on $\Omega_{t}$ are well-defined by setting $\mathbf{u}(x, t)=\mathbf{v}(y, t)$ and $\mathfrak{p}(x, t)=\mathfrak{q}(y, t)$. Since div $\mathbf{u}=0$ in $\Omega_{t},\left|\Omega_{t}\right|$ is a constant, and so $\left|\Omega_{t}\right|=\left|B_{R}\right|$ by assumption (1.5). Moreover, if we set

$$
\eta(t)=\frac{1}{\left|B_{R}\right|} \int_{\Omega_{t}} x \mathrm{~d} x,
$$

then

$$
\eta^{\prime}(t)=\frac{1}{\left|B_{R}\right|} \int_{\Omega_{t}} \mathbf{u}(x, t) \mathrm{d} x=\xi^{\prime}(t)
$$


and so $\eta(t)=\xi(t)+d$ with some constant $d$. We assume that the assumption (1.4) holds, and then by (2.18) we have

$$
0=\int_{0}^{2 \pi} \eta(t) \mathrm{d} t=2 \pi d+\int_{0}^{2 \pi} \xi(t) \mathrm{d} t=2 \pi d,
$$

which leads to $d=0$, that is

$$
\xi(t)=\frac{1}{\left|B_{R}\right|} \int_{\Omega_{t}} x \mathrm{~d} x .
$$

Combining this with (1.5) gives that

$$
\int_{S_{R}}(R+\rho)^{N} \mathrm{~d} \omega=0, \quad \int_{S_{R}}(R+\rho)^{N+1} \mathrm{~d} \omega=0,
$$

which yields that $\rho$ satisfies the equation:

$$
\partial_{t} \rho-\mathcal{A} \mathbf{v} \cdot \mathbf{n}=d(\mathbf{v}, \rho) \quad \text { on } S_{R} .
$$

Therefore, the kinematic equation: $V_{\Gamma_{t}}=\mathbf{u} \cdot \mathbf{n}_{t}$ holds on $\Gamma_{t}$. So far, we see that $\Omega_{t}$, $\mathbf{u}$ and $\mathfrak{p}$ satisfy equations (2.2). Since $D \subset B_{R}$, there exists a constant $\epsilon_{0}>0$ for which $D \subset B_{R-3 \epsilon_{0}}$. Since $\Omega_{t}$ is a small perturbation of $B_{R}$, choosing $\epsilon>0$ smaller if necessary, we may assume that $B_{R-\epsilon_{0}} \subset \Omega_{t}$, and so by (1.6) we have

$$
\int_{0}^{2 \pi}\left(\mathbf{f}(\cdot, t), \mathbf{p}_{\ell}\right)_{\Omega_{t}} \mathrm{~d} t=0 \text { for } \ell=1, \ldots, N .
$$

Multiplying the first equation in (2.2) with $\mathbf{p}_{\ell}$, integrating the resultant formulas with respect to $x$ on $\Omega_{t}$ and with respect to $t$ on $(0,2 \pi)$, and using the periodicity (1.2) and (2.19) we have

$$
\sum_{k=1}^{M} \int_{0}^{2 \pi}\left(\mathbf{u}(\cdot, t), \mathbf{p}_{k}\right)_{\Omega_{t}} \mathrm{~d} t \int_{0}^{2 \pi}\left(\mathbf{p}_{k}, \mathbf{p}_{\ell}\right)_{\Omega_{t}}=\int_{0}^{2 \pi}\left(\mathbf{f}(\cdot, t), \mathbf{p}_{\ell}\right)_{\Omega_{t}} \mathrm{~d} t=0
$$

for $\ell=1, \ldots, M$. Since $\Omega_{t}$ is a small perturbation of $B_{R}$, we may assume that the assumption (1.3) holds, and so by (2.20) we have

$$
\int_{0}^{2 \pi}\left(\mathbf{u}(\cdot, t), \mathbf{p}_{\ell}\right)_{\Omega_{t}} \mathrm{~d} t=0 \text { for } \ell=1, \ldots, M .
$$

Therefore, $\Omega_{t}$, $\mathbf{u}$ and $\mathfrak{p}$ satisfy equations (1.1), and so we see that Theorem 1 follows immediately from Theorem 4.

\subsection{Two-phase problem}

We now formulate problem (1.9) in the fixed domain. The idea is essentially the same as in the one-phase case. Let $\dot{\Omega}=\Omega \backslash S_{R}, \Omega_{+}=B_{R}$ and $\Omega_{-}=\Omega \backslash \overline{B_{R}}$. We define the barycenter point, $\xi(t)$, of $\Omega_{+t}$ by setting

$$
\xi(t)=\frac{1}{\left|B_{R}\right|} \int_{\Omega_{+t}} x \mathrm{~d} x,
$$


where we have used the fact that $\left|\Omega_{+t}\right|=\left|B_{R}\right|$, which follows from the assumption (1.12). By the Reynolds transport theorem, we see that

$$
\frac{d}{\mathrm{~d} t} \xi(t)=\frac{1}{\left|B_{R}\right|} \int_{\Omega_{t}} \mathbf{u}(x, t) \mathrm{d} x .
$$

Let $\rho(y, t)$ be an unknown periodic function with period $2 \pi$ such that

$$
\Gamma_{t}=\left\{x=y+\rho(y, t) \mathbf{n}+\xi(t) \mid y \in S_{R}\right\},
$$

where $S_{R}=\left\{x \in \mathbb{R}^{N}|| x \mid=R\right\}$ and $\mathbf{n}$ is the unit outer normal to $S_{R}$, that is $\mathbf{n}=y /|y|$ for $y \in S_{R}$.

In the following, we fix the method how to extend this to a transformation from $\dot{\Omega}$ to $\Omega_{t}$. Let $H$ be a unique solution of the Dirichlet problem:

$$
(1-\Delta) H_{\rho}=0 \quad \text { in } \mathbb{R}^{N} \backslash S_{R},\left.\quad H_{\rho}\right|_{S_{R}}=\rho .
$$

Let $L$ be a large number for which $\Omega \subset B_{L}$. From the $K$-method in real interpolation theory $[9,21]$, we see that

$$
\begin{aligned}
& C_{1}\left\|H_{\rho}(\cdot, t)\right\|_{H_{q}^{k}\left(\mathbb{R}^{N}\right)} \leq\|\rho(\cdot, t)\|_{W_{q}^{k-1 / q}\left(S_{R}\right)} \leq C_{2}\left\|H_{\rho}(\cdot, t)\right\|_{H_{q}^{k}\left(\mathbb{R}^{N}\right)} \text { for } k=1,2,3, \\
& C_{1}\left\|\partial_{t} H_{\rho}(\cdot, t)\right\|_{H_{q}^{k}\left(\mathbb{R}^{N}\right)} \leq\left\|\partial_{t} \rho(\cdot, t)\right\|_{W_{q}^{k-1 / q}\left(S_{R}\right)} \leq C_{2}\left\|\partial_{t} H_{\rho}(\cdot, t)\right\|_{H_{q}^{k}\left(\mathbb{R}^{N}\right)} \quad \text { for } k=1,2,
\end{aligned}
$$

for any $t \in(0,2 \pi)$. We may assume that there exists a small number $\omega>0$ for which $B_{R+3 \omega} \subset \Omega$. Let $\varphi$ be a function in $C^{\infty}\left(\mathbb{R}^{N}\right)$ for which equals one for $x \in$ $B_{R+\omega}$ and zero for $x \notin B_{R+2 \omega}$. Let $\Phi(y, t)=y+\varphi(y)\left(R^{-1} H_{\rho}(y, t) y+\xi(t)\right)$. Notice that $\Phi(y, t)=y+R^{-1} H_{\rho}(y, t) y+\xi(t)$ for $y \in B_{R}$. Setting $\Psi(y, t)=$ $\varphi(y)\left(R^{-1} H_{\rho}(y, t) y+\xi(t)\right)$, we assume that

$$
\sup _{t \in \mathbb{R}}\|\Psi(\cdot, t)\|_{H_{\infty}^{1}\left(\mathbb{R}^{N}\right)} \leq \delta
$$

with some small constant $\delta>0$. We choose $\delta>0$ so small that the map: $y \mapsto x=$ $\Phi(y, t)$ is bijective from $\Omega$ onto itself. In fact, for any $y_{1}$ and $y_{2}$

$$
\begin{aligned}
& \left|\Phi\left(y_{1}, t\right)-\Phi\left(y_{2}, t\right)\right| \geq\left|y_{1}-y_{2}\right| \\
& -\sup _{t \in \mathbb{R}}\|\nabla \Psi(\cdot, t)\|_{H_{\infty}^{1}\left(\mathbb{R}^{N}\right)}\left|y_{1}-y_{2}\right| \geq(1-\delta)\left|y_{1}-y_{2}\right|,
\end{aligned}
$$

which leads to the injectivity of the map: $x=\Phi(y, t)$ for any $t \in \mathbb{R}$ provided that $0<\delta<1$. Moreover, using the fact that $x=\Phi(y, t)=y$ for $y \in \Omega \backslash B_{R+2 \omega}$, and the inverse mapping theorem, we see that the map $x=\Phi(y, t)$ is surjective from $\Omega$ onto itself. Let

$$
\begin{aligned}
\Omega_{+t} & =\left\{x=\Phi(y, t)=y+R^{-1} H_{\rho}(y, t) y+\xi(t) \mid y \in B_{R}\right\}, \\
\Omega_{-t} & =\left\{x=\Phi(y, t)=y+\varphi(y)\left(R^{-1} H_{\rho}(y, t) y+\xi(t)\right) \mid y \in \Omega \backslash\left(S_{R} \cup B_{R}\right)\right\}, \\
\Gamma_{t} & =\left\{x=y+R^{-1} \rho(y, t) y+\xi(t) \mid y \in S_{R}\right\},
\end{aligned}
$$


Notice that $R^{-1} y$ is the unit outer normal to $S_{R}$ for $y \in S_{R}$. In the following, the jump quantity of $f$ defined on $\Omega \backslash S_{R}$ is also denoted by [[ $\left.\left.f\right]\right]$, which is defined by setting

$$
[[f]]\left(x_{0}, t\right)=\lim _{\substack{y \rightarrow x_{0} \\ y \in \Omega_{+}}} f(y, t)-\lim _{\substack{y \rightarrow x_{0} \\ y \in \Omega_{-}}} f(y, t) \text { for } x_{0} \in S_{R},
$$

where we have set $\Omega_{+}=B_{R}$ and $\Omega_{-}=\Omega \backslash\left(B_{R} \cup S_{R}\right)$. Let $\dot{\Omega}=\Omega_{+} \cup \Omega_{-}$, and for $f$ defined on $\dot{\Omega}$, we write $f_{ \pm}=\left.f\right|_{\Omega_{ \pm}}$. On the other hand, for $f_{ \pm}$defined on $\Omega_{ \pm}$, we define $f$ by $\left.f\right|_{\Omega_{ \pm}}=f_{ \pm}$.

Let $\mathbf{u}(x, t)$ and $\mathfrak{p}(x, t)$ satisfy the equations (1.9), and let $\Phi^{-1}(x, t)$ be the inverse map of $x=\Phi(y, t)$. Let $\mathbf{v}_{ \pm}(y, t)=\mathbf{u}_{ \pm}\left(\Phi^{-1}(y, t), t\right)$ and $\mathfrak{q}_{ \pm}(y, t)=$ $\mathfrak{p}_{ \pm}\left(\Phi^{-1}(y, t), t\right)$ for $y \in \Omega_{ \pm t}$. We derive an equation for $\mathbf{v}_{+}$and $\rho$ from the kinematic condition $V_{\Gamma_{t}}=\mathbf{u} \cdot \mathbf{n}_{t}$ on $\Gamma_{t}$. Noting that $[[\mathbf{u}]]=0$ on $\Gamma_{t}$, we may also assume that $[[\mathbf{v}]]=0$ on $S_{R}$, and so $\mathbf{v}_{+}=\mathbf{v}_{-}$on $S_{R}$.

From the definition it follows that

$$
V_{\Gamma_{t}}=\frac{\partial x}{\partial t} \cdot \mathbf{n}_{t}=\left(\frac{\partial \rho}{\partial t} \mathbf{n}+\xi^{\prime}(t)\right) \cdot \mathbf{n}_{t},
$$

Here and in the following, the unit outer normal to $S_{R}$ is denoted by $\mathbf{n}$, which is given by $\mathbf{n}(y)=R^{-1} y$ for $y \in S_{R}$. To represent the time derivative of $\xi(t)$ given in (2.21), we introduce the Jacobian $J_{+}(t)$ of the transformation: $x=y+R^{-1} H_{\rho} y+\xi(t)$ for $y \in B_{R}$, which is written as $J_{+}(t)=1+J_{0,+}(t)$ with

$$
J_{0,+}(t)=\operatorname{det}\left(\delta_{i j}+R^{-1} \frac{\partial}{\partial y_{i}}\left(H_{\rho}(y, t) y_{j}\right)\right)_{i, j=1, \ldots, N}-1 \text { for } y \in B_{R} .
$$

Choosing $\delta>0$ small enough in (2.24), we have

$$
\left\|J_{0,+}(t)\right\|_{L_{\infty}\left(B_{R}\right)} \leq C\left\|\nabla H_{\rho}(\cdot, t)\right\|_{L_{\infty}\left(B_{R}\right)} .
$$

From (2.21) it follows that

$$
\xi^{\prime}(t)=\frac{1}{\left|B_{R}\right|} \int_{B_{R}} \mathbf{v}_{+}(y, t) \mathrm{d} y+\frac{1}{\left|B_{R}\right|} \int_{B_{R}} \mathbf{v}_{+}(y, t) J_{0,+}(t) \mathrm{d} y
$$

and noting that $\mathbf{n} \cdot \mathbf{n}=1$, on $S_{R}$ we have the kinematic equation:

$$
\partial_{t} \rho-\left(\mathbf{v}-\frac{1}{\left|B_{R}\right|} \int_{B_{R}} \mathbf{v}_{+}(y, t) \mathrm{d} y\right) \cdot \mathbf{n}=d\left(\mathbf{v}_{+}, \rho\right)
$$

with

$d\left(\mathbf{v}_{+}, \rho\right)=\frac{1}{\left|B_{R}\right|} \int_{B_{R}} \mathbf{v}_{+}(y, t) J_{0,+}(t) \mathrm{d} y \cdot\left(\mathbf{n}-\mathbf{n}_{t}\right)+\frac{\partial \rho}{\partial t} \mathbf{n} \cdot\left(\mathbf{n}-\mathbf{n}_{t}\right)+\mathbf{v}_{+} \cdot\left(\mathbf{n}_{t}-\mathbf{n}\right)$.

As was already discussed in Sect. 2.1, from the assumption (1.12) and the representation formulas of $\Omega_{+t}$ and $\Gamma_{t}$ in (2.25), we have (2.12) in Sect. 2.1, too. Moreover, from (2.21) and the assumption (1.12), we have (2.13) in Sect. 2.1, too. Thus, under 
the assumption (1.12) and the representation of $\Gamma_{t}$ and $\Omega_{+t}$ in (2.25), the kinematic condition is equivalent to the equation:

$$
\begin{aligned}
& \partial_{t} \rho+\int_{S_{R}} \rho \mathrm{d} \omega+\sum_{k=1}^{N}\left(\int_{S_{R}} \rho \omega_{k} \mathrm{~d} \omega\right) y_{k} \\
& -\left(\mathbf{v}_{+}-\frac{1}{\left|B_{R}\right|} \int_{B_{R}} \mathbf{v}_{+} \mathrm{d} y\right) \cdot \mathbf{n}=\tilde{d}\left(\mathbf{v}_{+}, \rho\right) \text { on } S_{R} \times(0,2 \pi)
\end{aligned}
$$

with

$\tilde{d}\left(\mathbf{v}_{+}, \rho\right)=d\left(\mathbf{v}_{+}, \rho\right)-\sum_{k=2}^{N} \frac{{ }_{N} C_{k}}{N} \int_{S_{R}} R^{1-k} \rho^{k} \mathrm{~d} \omega-\sum_{k=2}^{N+1} \frac{N+1 C_{k}}{N+1} R^{1-k}\left(\int_{S_{R}} \rho^{k} \omega \mathrm{d} \omega\right) y_{k}$.

And then, to prove Theorem 3, we shall prove the global well-posedness of the following equations:

$$
\begin{cases}\partial_{t} \mathbf{v}_{ \pm}-\operatorname{Div}\left(\mu_{ \pm}\left(\mathbf{D}\left(\mathbf{v}_{ \pm}\right)-\mathfrak{q}_{ \pm}\right)=\mathbf{G}_{ \pm}+\mathbf{F}_{ \pm}(\mathbf{v}, \rho)\right. & \text { in } \Omega_{ \pm} \times(0,2 \pi), \\ \operatorname{div} \mathbf{v}_{ \pm}=g_{ \pm}(\mathbf{v}, \rho)=\operatorname{div} \mathbf{g}_{ \pm}(\mathbf{v}, \rho) & \text { in } \Omega_{ \pm} \times(0,2 \pi), \\ \partial_{t} \rho+\mathcal{M} \rho-\mathcal{A} \mathbf{v}_{+} \cdot \mathbf{n}=\tilde{d}\left(\mathbf{v}_{+}, \rho\right) & \text { on } S_{R} \times(0,2 \pi), \\ {\left[\left[\mu_{ \pm} \mathbf{D}\left(\mathbf{v}_{ \pm}\right)-\mathfrak{q}_{ \pm}\right]\right] \mathbf{n}-\left(\mathcal{B}_{R} \rho\right) \mathbf{n}=\tilde{\mathbf{h}}(\mathbf{v}, \rho)} & \text { on } S_{R} \times(0,2 \pi), \\ {[[\mathbf{v}]]=0} & \text { on } S_{R} \times(0,2 \pi), \\ \mathbf{v}_{-}=0 & \text { on } S \times(0,2 \pi),\end{cases}
$$

where we have set

$$
\mathcal{A} \mathbf{v}_{+}=\mathbf{v}_{+}-\frac{1}{\left|B_{R}\right|} \int_{B_{R}} \mathbf{v}_{+} \mathrm{d} y
$$

and $\mathcal{M} \rho$ and $\mathcal{B}_{R} \rho$ are the same as in (2.17) in Sect. 2.1. For the functions on the right side of equations (2.31), $\mathbf{G}_{ \pm}$and $\mathbf{F}_{ \pm}(\mathbf{v}, \rho)$ are defined in (3.39) of Sect. 3, $g_{ \pm}(\mathbf{v}, \rho)$ and $\mathbf{g}_{ \pm}(\mathbf{v}, \rho)$ are defined in (3.38) of Sect. 3, and $\tilde{\mathbf{h}}(\mathbf{v}, \rho)$ is defined in (3.40) of Sect. 3

The following theorem is the unique existence theorem of $2 \pi$-periodic solutions of problem (2.31).

Theorem 5. Let $1<p, q<\infty$ and $2 / p+N / q<1$. Then, there exists a small constant $\epsilon>0$ such that for any $\mathbf{f} \in L_{p, \operatorname{per}}\left((0,2 \pi), L_{q}(\Omega)^{N}\right)$ satisfying the smallness condition: $\|\mathbf{f}\|_{L_{p}\left((0,2 \pi), L_{q}(\Omega)\right)} \leq \epsilon$, problem $(2.31)$ admits solutions $\mathbf{v}_{ \pm}, \mathfrak{q}_{ \pm}$, and $\rho$ satisfying the regularity condition (1.13) and the estimate (1.14) in Theorem 3.

Employing the same argument as in the proof of Theorem 1 in Sect. 2.1, we see that Theorem 3 immediately follows from Theorem 5. 


\section{Derivation of nonlinear terms}

\subsection{One-phase problem case}

First, we consider the one-phase problem case and we consider the map

$$
x=y+\Psi(y, t),
$$

where $\Psi(y, t)=R^{-1} H_{\rho}(y, t) y+\xi(t)$ and $H_{\rho}$ satisfies the condition (2.5) and (2.6). Recall that $H_{\rho}(y, t)=\rho(y, t)$ for $y \in S_{R}$. Let $\Omega_{t}, \Gamma_{t}, \mathbf{u}(x, t)$ and $\mathfrak{p}(x, t)$ satisfy the equations (1.1) and

$\Omega_{t}=\left\{x=y+\Psi(y, t) \mid y \in B_{R}\right\}, \quad \Gamma_{t}=\left\{x=y+R^{-1} \rho(y, t) y+\xi(t) \mid y \in S_{R}\right\}$.

Choose $\delta>0$ small in such a way that there exists an inverse map: $y=\Phi^{-1}(x, t)$ of the map: $x=\Phi(y, t)=y+\Psi(y, t)$. Let $\mathbf{v}(y, t)=\mathbf{u}\left(\Phi^{-1}(y, t), t\right)$ and $\mathfrak{q}(y, t)=$ $\mathfrak{p}\left(\Phi^{-1}(y, t), t\right)$. By the chain rule, we have

$$
\nabla_{x}=\left(\mathbf{I}+\mathbf{V}_{0}(\mathbf{k})\right) \nabla_{y}, \quad \frac{\partial}{\partial x_{i}}=\frac{\partial}{\partial y_{i}}+\sum_{j=1}^{N} V_{0 i j}(\mathbf{k}) \frac{\partial}{\partial y_{j}}
$$

where $\nabla_{z}={ }^{\top}\left(\partial / \partial z_{1}, \ldots, \partial / \partial z_{N}\right)$ for $z \in\{x, y\}$ and $\mathbf{k}=\left(k_{0}, k_{1}, \ldots, k_{N}\right)=$ $\left(H_{\rho}, \nabla H_{\rho}\right)$. Here, $\mathbf{V}_{0}(\mathbf{k})$ is an $(N \times N)$-matrix of $C^{\infty}$ functions defined for $|\mathbf{k}| \leq \delta$ with $\mathbf{V}_{0}(0)=0$ and $V_{0 i j}(\mathbf{k})$ is the $(i, j)$ th component of $\mathbf{V}_{0}(\mathbf{k})$. By (3.2), we can write $\mathbf{D}(\mathbf{u})$ as $\mathbf{D}(\mathbf{u})=\mathbf{D}(\mathbf{v})+\mathcal{D}_{\mathbf{D}}(\mathbf{k}) \nabla \mathbf{v}$ with

$$
\begin{gathered}
\mathbf{D}(\mathbf{v})_{i j}=\frac{\partial v_{i}}{\partial y_{j}}+\frac{\partial v_{j}}{\partial y_{i}}, \\
\left(\mathcal{D}_{\mathbf{D}}(\mathbf{k}) \nabla \mathbf{v}\right)_{i j}=\sum_{k=1}^{N}\left(V_{0 j k}(\mathbf{k}) \frac{\partial v_{i}}{\partial y_{k}}+V_{0 i k}(\mathbf{k}) \frac{\partial v_{j}}{\partial y_{k}}\right) .
\end{gathered}
$$

We next consider div v. By (3.2), we have

$$
\operatorname{div}_{x} \mathbf{u}=\sum_{j=1}^{N} \frac{\partial u_{j}}{\partial x_{j}}=\sum_{j, k=1}^{N}\left(\delta_{j k}+V_{0 j k}(\mathbf{k})\right) \frac{\partial v_{j}}{\partial y_{k}}=\operatorname{div}_{y} \mathbf{v}+\mathbf{V}_{0}(\mathbf{k}): \nabla \mathbf{v}
$$

Let $J$ be the Jacobian of the transformation (3.1). Choosing $\delta>0$ small enough, we may assume that $J=J(\mathbf{k})=1+J_{0}(\mathbf{k})$, where $J_{0}(\mathbf{k})$ is a $C^{\infty}$ function defined for $|\mathbf{k}|<\sigma$ such that $J_{0}(0)=0$.

To obtain another representation formula of $\operatorname{div} x \mathbf{u}$, we use the inner product $(\cdot, \cdot)_{\Omega_{t}}$. For any test function $\varphi \in C_{0}^{\infty}\left(\Omega_{t}\right)$, we set $\psi(y)=\varphi(x)$. We then have

$$
\begin{aligned}
& \left(\operatorname{div}_{x} \mathbf{u}, \varphi\right)_{\Omega_{t}}=-(\mathbf{u}, \nabla \varphi)_{\Omega_{t}}=-\left(J \mathbf{v},\left(\mathbf{I}+\mathbf{V}_{0}\right) \nabla_{y} \psi\right)_{\Omega} \\
& \quad=\left(\operatorname{div}\left(\left(\mathbf{I}+{ }^{\top} \mathbf{V}_{0}\right) J \mathbf{v}\right), \psi\right)_{\Omega}=\left(J^{-1} \operatorname{div}\left(\left(\mathbf{I}+{ }^{\top} \mathbf{V}_{0}\right) J \mathbf{v}\right), \varphi\right)_{\Omega_{t}},
\end{aligned}
$$


which, combined with (3.4), leads to

$$
\operatorname{div}_{x} \mathbf{u}=\operatorname{div}_{y} \mathbf{v}+\mathbf{V}_{0}(\mathbf{k}): \nabla \mathbf{v}=J^{-1}\left(\operatorname{div}_{y} \mathbf{v}+\operatorname{div}_{y}\left(J^{\top} \mathbf{V}_{0}(\mathbf{k}) \mathbf{v}\right)\right) .
$$

Recalling that $J=J(\mathbf{k})=1+J_{0}(\mathbf{k})$, we define $g(\mathbf{v}, \rho)$ and $\mathbf{g}(\mathbf{v}, \rho)$ by letting

$$
\begin{aligned}
& g(\mathbf{v}, \rho)=-\left(J_{0}(\mathbf{k}) \operatorname{div} \mathbf{v}+\left(1+J_{0}(\mathbf{k})\right) \mathbf{V}_{0}(\mathbf{k}): \nabla \mathbf{v}\right), \\
& \mathbf{g}(\mathbf{v}, \rho)=-\left(1+J_{0}(\mathbf{k})\right)^{\top} \mathbf{V}_{0}(\mathbf{k}) \mathbf{v},
\end{aligned}
$$

and then by (3.5) we see that the divergence free condition: div $\mathbf{u}=0$ is transformed to the second equation in the equations (2.16). In particular, it follows from (3.5) that

$$
J_{0}(k) \operatorname{div} \mathbf{v}+J(k) \mathbf{V}_{0}(\mathbf{k}): \nabla \mathbf{v}=\operatorname{div}\left(J(k)^{\top} \mathbf{V}_{0}(\mathbf{k}) \mathbf{v}\right)
$$

To derive $\mathbf{F}(\mathbf{v}, \rho)$, we first observe that

$$
\begin{aligned}
& \sum_{j=1}^{N} \frac{\partial}{\partial x_{j}}\left(\mu \mathbf{D}(\mathbf{u})_{i j}-\mathfrak{p} \delta_{i j}\right) \\
& \quad=\sum_{j, k=1}^{N} \mu\left(\delta_{j k}+V_{0 j k}\right) \frac{\partial}{\partial y_{k}}\left(\mathbf{D}(\mathbf{v})_{i j}+\left(\mathcal{D}_{\mathbf{D}}(\mathbf{k}) \nabla \mathbf{v}\right)_{i j}\right)-\sum_{j=1}^{N}\left(\delta_{i j}+V_{0 i j}\right) \frac{\partial \mathfrak{q}}{\partial y_{j}}
\end{aligned}
$$

where we have used (3.3). Since

$$
\frac{\partial}{\partial t}\left[u_{i}(y+\Psi(y, t), t)\right]=\frac{\partial u_{i}}{\partial t}(x, t)+\sum_{j=1}^{N} \frac{\partial \Psi_{j}}{\partial t} \frac{\partial u_{i}}{\partial x_{j}}(x, t),
$$

we have

$$
\frac{\partial u_{i}}{\partial t}=\frac{\partial v_{i}}{\partial t}-\sum_{j, k=1}^{N} \frac{\partial \Psi_{j}}{\partial t}\left(\delta_{j k}+V_{0 j k}\right) \frac{\partial v_{i}}{\partial y_{k}}
$$

and therefore,

$$
\frac{\partial u_{i}}{\partial t}+\sum_{j=1}^{N} u_{j} \frac{\partial u_{i}}{\partial x_{j}}=\frac{\partial v_{i}}{\partial t}+\sum_{j, k=1}^{N}\left(v_{j}-\frac{\partial \Psi_{j}}{\partial t}\right)\left(\delta_{j k}+V_{0 j k}(\mathbf{k})\right) \frac{\partial v_{i}}{\partial y_{k}} .
$$

Putting (3.8) and (3.9) together gives

$$
\begin{aligned}
f_{i}(x, t)= & \left(\frac{\partial v_{i}}{\partial t}+\sum_{j, k=1}^{N}\left(v_{j}-\frac{\partial \Psi_{j}}{\partial t}\right)\left(\delta_{j k}+V_{0 j k}(\mathbf{k})\right) \frac{\partial v_{i}}{\partial y_{k}}\right) \\
& -\mu \sum_{j, k=1}^{N}\left(\delta_{j k}+V_{0 j k}(\mathbf{k})\right) \frac{\partial}{\partial y_{k}}\left(\mathbf{D}(\mathbf{v})_{i j}+\left(\mathcal{D}_{\mathbf{D}}(\mathbf{k}) \nabla \mathbf{v}\right)_{i j}\right)
\end{aligned}
$$




$$
-\sum_{j=1}^{N}\left(\delta_{i j}+V_{0 i j}(\mathbf{k})\right) \frac{\partial \mathfrak{q}}{\partial y_{j}} .
$$

Since $(\mathbf{I}+\nabla \Psi)\left(\mathbf{I}+\mathbf{V}_{0}\right)=(\partial x / \partial y)(\partial y / \partial x)=\mathbf{I}$, that is,

$$
\sum_{i=1}^{N}\left(\delta_{m i}+\partial_{m} \Psi_{i}\right)\left(\delta_{i j}+V_{0 i j}(\mathbf{k})\right)=\delta_{m j},
$$

we have

$$
\begin{aligned}
& \sum_{i=1}^{N}\left(\delta_{m i}+\partial_{m} \Psi_{i}\right) f_{i}(\Psi(y, t), t) \\
& =\sum_{i=1}^{N}\left(\delta_{m i}+\partial_{m} \Psi_{i}\right)\left(\frac{\partial v_{i}}{\partial t}+\sum_{j, k=1}^{N}\left(v_{j}-\frac{\partial \Psi_{i}}{\partial t}\right)\left(\delta_{j k}+V_{0 j k}(\mathbf{k})\right) \frac{\partial v_{i}}{\partial y_{k}}\right) \\
& \quad-\mu \sum_{i, j, k=1}^{N}\left(\delta_{m i}+\partial_{m} \Psi_{i}\right)\left(\delta_{j k}+V_{0 j k}(\mathbf{k})\right) \frac{\partial}{\partial y_{k}}\left(\mathbf{D}(\mathbf{v})_{i j}+\left(\mathcal{D}_{\mathbf{D}}(\mathbf{k}) \nabla \mathbf{v}\right)_{i j}\right)-\frac{\partial \mathfrak{q}}{\partial y_{m}} .
\end{aligned}
$$

Thus, changing $i$ to $\ell$ and $m$ to $i$ in the formula above, we define an $N$-vector of functions $\mathbf{F}_{1}(\mathbf{v}, \rho)$ by letting

$$
\begin{aligned}
& \left.\mathbf{F}_{1}(\mathbf{v}, \rho)\right|_{i}=-\sum_{j, k=1}^{N}\left(v_{j}-\frac{\partial \Psi_{j}}{\partial t}\right)\left(\delta_{j k}+V_{0 j k}(\mathbf{k})\right) \frac{\partial v_{i}}{\partial y_{k}} \\
& -\sum_{\ell=1}^{N} \partial_{i} \Psi_{\ell}\left(\frac{\partial v_{\ell}}{\partial t}+\sum_{j, k=1}^{N}\left(v_{j}-\frac{\partial \Psi_{j}}{\partial t}\right)\left(\delta_{j k}+V_{0 j k}(\mathbf{k})\right) \frac{\partial v_{\ell}}{\partial y_{k}}\right) \\
& +\mu\left(\sum_{j=1}^{N} \frac{\partial}{\partial y_{j}}\left(\mathcal{D}_{\mathbf{D}}(\mathbf{k}) \nabla \mathbf{v}\right)_{i j}+\sum_{j, k=1}^{N} V_{0 j k}(\mathbf{k}) \frac{\partial}{\partial y_{k}}\left(\mathbf{D}(\mathbf{v})_{i j}+\left(\mathcal{D}_{\mathbf{D}}(\mathbf{k}) \nabla \mathbf{v}\right)_{i j}\right)\right. \\
& \left.+\sum_{j, k, \ell=1}^{N} \partial_{i} \Psi_{\ell}\left(\delta_{j k}+V_{0 j k}(\mathbf{k})\right) \frac{\partial}{\partial y_{k}}\left(\mathbf{D}(\mathbf{v})_{\ell j}+\left(\mathcal{D}_{\mathbf{D}}(\mathbf{k}) \nabla \mathbf{v}\right)_{\ell j}\right)\right),
\end{aligned}
$$

where $\left.\mathbf{F}_{1}(\mathbf{u}, \rho)\right|_{i}$ denotes the $i$ th component of $\mathbf{F}_{1}(\mathbf{u}, \rho)$.

Moreover,

$$
\begin{aligned}
(\mathbf{I} & +\nabla \Psi) \sum_{k=1}^{M} \int_{0}^{2 \pi}\left(\mathbf{u}(\cdot, t), \mathbf{p}_{k}(\cdot)\right)_{\Omega_{t}} \mathrm{~d} t \mathbf{p}_{k}(x) \\
& =(\mathbf{I}+\nabla \Psi) \sum_{k=1}^{M} \int_{0}^{2 \pi} \int_{B_{R}}\left(\mathbf{v}(y, t) \cdot \mathbf{p}_{k}(y+\Psi(y, t))\left(1+J_{0}(t)\right) \mathrm{d} y \mathrm{~d} t \mathbf{p}_{k}(y+\Psi(y, t))\right. \\
& =\mathcal{L} \mathbf{v}_{S}+\mathbf{F}_{2}(\mathbf{v}, \rho)
\end{aligned}
$$


with

$$
\begin{aligned}
\mathbf{F}_{2}(\mathbf{v}, \rho)= & \sum_{k=1}^{M}\left\{\int _ { 0 } ^ { 2 \pi } \int _ { B _ { R } } \left(\mathbf { v } ( y , t ) \cdot \left(\mathbf{p}_{k}(y) J_{0}(t)+\tilde{\mathbf{p}}_{k}(\Psi(y, t))\left(1+J_{0}(t)\right) \mathrm{d} y \mathrm{~d} t \mathbf{p}_{k}(y)\right.\right.\right. \\
& +\int_{0}^{2 \pi} \int_{B_{R}} \mathbf{v}(y, t) \cdot \mathbf{p}_{k}(y+\Psi(y, t))\left(1+J_{0}(t)\right) \mathrm{d} y \mathrm{~d} t \tilde{\mathbf{p}}_{k}(\Psi(y, t)) \\
& +\nabla \Psi \int_{0}^{2 \pi} \int_{B_{R}} \mathbf{v}(y, t) \cdot \mathbf{p}_{k}(y+\Psi(y, t))\left(1+J_{0}(t)\right) \mathrm{d} y \mathrm{~d} t \mathbf{p}_{k}(y+\Psi(y, t)),
\end{aligned}
$$

where we have set

$$
\tilde{\mathbf{p}}_{k}(\Psi(y, t))= \begin{cases}0 & \text { for } k=1, \ldots, N \\ c_{i j}\left(\Psi_{i}(y, t) \mathbf{e}_{j}-\Psi_{j}(y, t) \mathbf{e}_{i}\right) & \text { for } k=N+1, \ldots, M\end{cases}
$$

Thus, setting

$$
\mathbf{G}(y, t)=(\mathbf{I}+\nabla \Psi(y, t)) \mathbf{f}(y+\Psi(y, t), t), \quad \mathbf{F}(\mathbf{v}, \rho)=\mathbf{F}_{1}(\mathbf{v}, \rho)+\mathbf{F}_{2}(\mathbf{v}, \rho),
$$

we have the first equation in equations (2.16).

We next consider the transformation of the boundary conditions. Recall that $\Gamma_{t}$ is represented by $x=y+\rho(y, t) \mathbf{n}(y)+\xi(t)$ for $y \in S_{R}$ with $\mathbf{n}(y)=y /|y|$. Let $x_{0}$ be any point on $S_{R}$ and let $\Phi(p)$ be a $C^{\infty}$ diffeomorphism on $\mathbb{R}^{N}$ such that—up to a rotation-it holds

$$
B_{R} \cap B_{\omega}\left(x_{0}\right)=\Phi\left(\left\{p \in \mathbb{R}^{N}\left|0<p_{N}<\omega, \quad\right|\left|p^{\prime}\right|<\omega\right\}\right) \cap B_{\omega}\left(x_{0}\right),
$$

where we have set $B_{\omega}\left(x_{0}\right)=\left\{y \in \mathbb{R}^{N}|| y-x_{0} \mid<\omega\right\}$ and $p^{\prime}=\left(p_{1}, \ldots, p_{N-1}\right)$. Notice that $y=\Phi\left(p^{\prime}, 0\right) \in S_{R} \cap B_{\omega}\left(x_{0}\right)$ and $\rho(y, t)=H_{\rho}\left(\Phi\left(p^{\prime}, 0\right), t\right)$. Let $\left\{x_{k}\right\}_{k=1}^{K}$ and $\left\{\zeta_{k}\right\}_{k=1}^{K}$ be a finite number of points on $S_{R}$ and a partition of unity of $S_{R}$ such that $\operatorname{supp} \zeta_{k} \subset B_{\omega}\left(x_{k}\right)$ and $\sum_{k=1}^{K} \zeta_{k}(y)=1$ on $S_{R}$. In the following, we represent functions on each $S_{R} \cap B_{\omega}\left(x_{k}\right)$, and to represent functions globally, we use the formula:

$$
f=\sum_{k=1}^{K} \zeta_{k}^{1} f \quad \text { in } S_{R}
$$

Thus, for the detailed calculations, we only consider the domain $B_{R} \cap B_{\omega}\left(x_{\ell}\right)(\ell=$ $1, \ldots, K)$, and use the local coordinate system: $y=\Phi_{\ell}(p)$ for $p \in U$, where we have written $\Phi=\Phi_{\ell}$, and $U=\left\{p \in \mathbb{R}^{N}\left|0<p_{N}<\omega,\right| p^{\prime} \mid<\omega\right\}$.

We write $\rho=\rho\left(y\left(p_{1}, \ldots, p_{N-1}, 0\right), t\right)$ in the following. By the chain rule, we have

$$
\frac{\partial \rho}{\partial p_{i}}=\frac{\partial}{\partial p_{i}} H_{\rho}\left(\Phi_{\ell}\left(p_{1}, \ldots, p_{N-1}, 0\right), t\right)=\left.\sum_{m=1}^{N} \frac{\partial H_{\rho}}{\partial y_{m}} \frac{\partial \Phi_{\ell, m}}{\partial p_{i}}\right|_{p_{N}=0},
$$


where we have set $\Phi_{\ell}={ }^{\top}\left(\Phi_{\ell, 1}, \ldots, \Phi_{\ell, N}\right)$, and so, $\partial \rho / \partial p_{i}$ is defined in $B_{\omega}\left(x_{0}\right)$ by letting

$$
\frac{\partial \rho}{\partial p_{i}}=\sum_{m=1}^{N} \frac{\partial H_{\rho}}{\partial y_{m}} \circ \Phi_{\ell} \frac{\partial \Phi_{\ell, m}}{\partial p_{i}} .
$$

We first represent $\mathbf{n}_{t}$. Since $\Gamma_{t}$ is given by $x=y+\rho(y, t) \mathbf{n}+\xi(t)$ for $y \in S_{R}$,

$$
\mathbf{n}_{t}=a\left(\mathbf{n}+\sum_{i=1}^{N-1} b_{i} \tau_{i}\right) \quad \text { with } \tau_{i}=\frac{\partial}{\partial p_{i}} y=\frac{\partial}{\partial p_{i}} \Phi_{\ell}\left(p^{\prime}, 0\right)
$$

The vectors $\tau_{i}(i=1, \ldots, N-1)$ form a basis of the tangent space of $S_{R}$ at $y=$ $y\left(p_{1}, \ldots, p_{N-1}\right)$. Since $\left|\mathbf{n}_{t}\right|^{2}=1$, we have

$$
1=a^{2}\left(1+\sum_{i, j=1}^{N-1} g_{i j} b_{i} b_{j}\right) \quad \text { with } g_{i j}=\tau_{i} \cdot \tau_{j}
$$

because $\tau_{i} \cdot \mathbf{n}=0$. The vectors $\frac{\partial x}{\partial p_{i}}(i=1, \ldots, N-1)$ form a basis of the tangent space of $\Gamma_{t}$, and so $\mathbf{n}_{t} \cdot \frac{\partial x}{\partial p_{i}}=0$. Thus, we have

$$
0=a\left(\mathbf{n}+\sum_{j=1}^{N-1} b_{j} \tau_{j}\right) \cdot\left(\frac{\partial y}{\partial p_{i}}+\frac{\partial \rho}{\partial p_{i}} \mathbf{n}+\rho \frac{\partial \mathbf{n}}{\partial p_{i}}\right)
$$

Since $\mathbf{n} \cdot \frac{\partial y}{\partial p_{i}}=\mathbf{n} \cdot \tau_{i}=0, \frac{\partial \mathbf{n}}{\partial p_{i}} \cdot \mathbf{n}=0$ (because of $|\mathbf{n}|^{2}=1$ ), and $\frac{\partial y}{\partial p_{i}} \cdot \frac{\partial y}{\partial p_{j}}=$ $\tau_{i} \cdot \tau_{j}=g_{i j}$, recalling that $\mathbf{n}=R^{-1} y=R^{-1} \Phi_{\ell}$, by (3.18) we have

$$
\frac{\partial \rho}{\partial p_{i}}+\sum_{j=1}^{N-1}\left(1+R^{-1} \rho\right) g_{i j} b_{j}=0
$$

Let $G=\left(g_{i j}\right)$ and $G^{-1}=\left(g^{i j}\right)$, and then setting $\nabla_{\Gamma}^{\prime} \rho=\left(\partial \rho / \partial p_{1}, \ldots, \partial \rho / \partial p_{N-1}\right)$, we have

$$
b_{i}=-\left(1+R^{-1} \rho\right)^{-1} \sum_{k=1}^{N-1} g^{i k} \frac{\partial \rho}{\partial p_{k}}, \quad b=-\left(1+R^{-1} \rho\right)^{-1} G^{-1} \nabla_{\Gamma}^{\prime} \rho,
$$

which leads to

$$
\mathbf{n}_{t}=a\left(\mathbf{n}-\left(1+R^{-1} \rho\right)^{-1} \sum_{i, j=1}^{N-1} g^{i j} \frac{\partial \rho}{\partial p_{j}} \tau_{i}\right) .
$$

Moreover, combining (3.17) and (3.19), we have

$$
a=\left(1+\left(1+R^{-1} \rho\right)^{-2}<G^{-1} \nabla_{\Gamma}^{\prime} \rho, \nabla_{\Gamma}^{\prime} \rho>\right)^{-1 / 2} .
$$


Using the formula:

$$
(1+f)^{-1 / 2}=1-\frac{1}{2} \int_{0}^{1}(1+\theta f)^{-3 / 2} \mathrm{~d} \theta f
$$

we have

$$
a=1-V_{\Gamma}\left(\rho, \nabla_{\Gamma}^{\prime} \rho\right)
$$

with

$$
\begin{aligned}
V_{\Gamma}\left(\rho, \nabla_{\Gamma}^{\prime} \rho\right) & =\frac{1}{2} \int_{0}^{1}\left(1+\theta\left(1+R^{-1} \rho\right)^{-2}<G^{-1} \nabla_{\Gamma}^{\prime} \rho, \nabla_{\Gamma}^{\prime} \rho>\right)^{-3 / 2} \mathrm{~d} \theta\left(1+R^{-1} \rho\right)^{-2} \\
& <G^{-1} \nabla_{\Gamma}^{\prime} \rho, \nabla_{\Gamma}^{\prime} \rho>
\end{aligned}
$$

Combining these formulas obtained above gives

$$
\mathbf{n}_{t}=\mathbf{n}-\sum_{i, j=1}^{N-1} g^{i j} \frac{\partial \rho}{\partial p_{j}} \tau_{i}+\mathbf{V}_{\mathbf{n}}\left(\rho, \nabla_{\Gamma}^{\prime} \rho\right)
$$

where we have set

$$
\begin{aligned}
& \mathbf{V}_{\mathbf{n}}\left(\rho, \nabla_{\Gamma}^{\prime} \rho\right)=\frac{\rho}{R+\rho} \sum_{i, j=1}^{N-1} g^{i j} \frac{\partial \rho}{\partial p_{j}} \tau_{i} \\
& -\left(\mathbf{n}-\sum_{i, j=1}^{N-1}\left(1+R^{-1} \rho\right)^{-1} g^{i j} \frac{\partial \rho}{\partial p_{j}} \tau_{i}\right) V_{\Gamma}\left(\rho, \nabla_{\Gamma}^{\prime} \rho\right) .
\end{aligned}
$$

From (3.16), $\nabla_{\Gamma}^{\prime} \rho$ is extended to $\mathbb{R}^{N}$ by the formula: $\nabla_{\Gamma}^{\prime} \rho=\left(\nabla \Phi_{\ell}\right) \nabla \Psi_{\rho} \circ \Phi_{\ell}$, and so we may write

$$
\mathbf{V}_{\mathbf{n}}\left(\rho, \nabla_{\Gamma}^{\prime} \rho\right)=\mathbf{V}_{\mathbf{n}, \ell}(\mathbf{k}) \bar{\nabla} \Psi_{\rho} \otimes \bar{\nabla} \Psi_{\rho}
$$

on $B_{\omega}\left(x_{\ell}\right)$ with some function $\mathbf{V}_{\mathbf{n}, \ell}(\mathbf{k})=\mathbf{V}_{\mathbf{n}, \ell}(y, \mathbf{k})$ defined on $B_{\omega}\left(x_{\ell}\right) \times\{\mathbf{k}|| \mathbf{k} \mid \leq \delta\}$ with $\mathbf{V}_{\mathbf{n}, \ell}(0)=0$ possessing the estimate

$$
\left\|\left(\mathbf{V}_{\mathbf{n}, \ell}(\cdot, \mathbf{k}), \partial_{\mathbf{k}} \mathbf{V}_{\mathbf{n}, \ell}(\cdot, \mathbf{k})\right)\right\|_{H_{\infty}^{1}\left(B_{\omega}\left(x_{\ell}\right)\right)} \leq C
$$

with some constant $C$ independent of $\ell$. Here and in the following $\mathbf{k}$ are the variables corresponding to $\bar{\nabla} H_{\rho}=\left(H_{\rho}, \nabla H_{\rho}\right)$. In view of (3.21), we have

$$
\mathbf{n}_{t}=\mathbf{n}-\sum_{i, j=1}^{N-1} g^{i j} \tau_{i} \frac{\partial \rho}{\partial p_{j}}+\mathbf{V}_{\mathbf{n}, \ell}(\mathbf{k}) \bar{\nabla} \Psi_{\rho} \otimes \bar{\nabla} \Psi_{\rho} \quad \text { on } B_{\omega}\left(x_{\ell}\right) \cap S_{R} .
$$

Thus, in view of (3.14) and (3.16), we may write

$$
\mathbf{n}_{t}=\mathbf{n}-\sum_{i, j=1}^{N-1} g^{i j} \partial_{j}^{\prime} \rho \tau_{i}+\mathbf{V}_{\mathbf{n}}\left(\bar{\nabla} H_{\rho}\right) \bar{\nabla} H_{\rho} \otimes \bar{\nabla} H_{\rho} \quad \text { on } S_{R},
$$


where $\partial_{j}^{\prime} \rho=\partial \rho / \partial p_{j}$ locally on $B_{\omega}\left(x_{\ell}\right) \cap S_{R}, \bar{\nabla} H_{\rho}=\left(H_{\rho}, \nabla H_{\rho}\right)$, and $\mathbf{V}_{\mathbf{n}}(\mathbf{k})$ is a matrix of functions defined on $\overline{B_{R}} \times\{\mathbf{k}|| \mathbf{k} \mid<\delta\}$ possessing the estimate:

$$
\left\|\left(\mathbf{V}_{\mathbf{n}}, \partial_{\mathbf{k}} \mathbf{V}_{\mathbf{n}}\right)(\cdot, \mathbf{k})\right\|_{H_{\infty}^{1}\left(B_{R}\right)} \leq C \text { for }|\overline{\mathbf{k}}| \leq \delta
$$

And also we may write

$$
\mathbf{n}_{t}=\mathbf{n}+\tilde{\mathbf{V}}_{\mathbf{n}}\left(\bar{\nabla} H_{\rho}\right) \bar{\nabla} H_{\rho}
$$

where $\tilde{\mathbf{V}}_{\mathbf{n}}(\mathbf{k})$ is a matrix of functions defined on $\overline{B_{R}} \times\{\mathbf{k}|| \mathbf{k} \mid<\delta\}$ possessing the estimate:

$$
\left\|\left(\tilde{\mathbf{V}}_{\mathbf{n}}(\cdot, \mathbf{k}), \partial_{\mathbf{k}} \tilde{\mathbf{V}}_{\mathbf{n}}(\cdot, \mathbf{k})\right)\right\|_{H_{\infty}^{1}\left(B_{R}\right)} \leq C \text { for }|\mathbf{k}| \leq \delta
$$

We now consider the boundary condition:

$$
(\mu \mathbf{D}(\mathbf{u})-\mathfrak{p I}) \mathbf{n}_{t}=\sigma H\left(\Gamma_{t}\right) \mathbf{n}_{t}-p_{0} \mathbf{n}_{t}
$$

It is convenient to divide the formula in (3.27) into the tangential part and normal part on $\Gamma_{t}$ as follows:

$$
\begin{gathered}
\Pi_{t} \mu \mathbf{D}(\mathbf{u}) \mathbf{n}_{t}=0, \\
<\mu \mathbf{D}(\mathbf{v}) \mathbf{n}_{t}, \mathbf{n}_{t}>-\mathfrak{p}=\sigma<H\left(\Gamma_{t}\right) \mathbf{n}_{t}, \mathbf{n}_{t}>-p_{0}=h_{N}(\mathbf{v}, \rho)
\end{gathered}
$$

Here, $\Pi_{t}$ is defined by $\Pi_{t} \mathbf{d}=\mathbf{d}-<\mathbf{d}, \mathbf{n}_{t}>\mathbf{n}_{t}$ for any $N$-vector of functions $\mathbf{d}$. In the last equation in equations (2.16), we set $\mathbf{h}^{\prime}(\mathbf{v}, \rho)=\mathbf{h}(\mathbf{v}, \rho)-<\mathbf{h}(\mathbf{v}, \rho), \mathbf{n}>\mathbf{n}$ and $h_{N}(\mathbf{v}, \rho)=<\mathbf{h}(\mathbf{v}, \rho), \mathbf{n}>$. By (3.25) and (3.3), we see that the boundary condition (3.28) is transformed to the following formula:

$$
(\mu \mathbf{D}(\mathbf{v}) \mathbf{n})_{\tau}=\mathbf{h}^{\prime}(\mathbf{v}, \rho) \text { on } \Gamma \times(0, T)
$$

where we have set $\mathbf{d}_{\tau}=\mathbf{d}-<\mathbf{d}, \mathbf{n}>\mathbf{n}$ and

$$
\begin{aligned}
& \mathbf{h}^{\prime}(\mathbf{v}, \rho)=-\mu \mathbf{D}(\mathbf{v}) \tilde{\mathbf{V}}_{\mathbf{n}}\left(\bar{\nabla} H_{\rho}\right) \bar{\nabla} H_{\rho} \\
& \quad+\mu\left\{<\mathbf{D}(\mathbf{v}) \tilde{\mathbf{V}}_{\mathbf{n}}\left(\bar{\nabla} H_{\rho}\right) \bar{\nabla} H_{\rho}, \mathbf{n}+\tilde{\mathbf{V}}_{\mathbf{n}}\left(\bar{\nabla} H_{\rho}\right) \bar{\nabla} H_{\rho}>\left(\mathbf{n}+\tilde{\mathbf{V}}_{\mathbf{n}}\left(\bar{\nabla} H_{\rho}\right) \bar{\nabla} H_{\rho}\right)\right. \\
& \quad+<\mathbf{D}(\mathbf{v}) \mathbf{n}, \tilde{\mathbf{V}}_{\mathbf{n}}\left(\bar{\nabla} H_{\rho}\right) \bar{\nabla} H_{\rho}>\left(\mathbf{n}+\tilde{\mathbf{V}}_{\mathbf{n}}\left(\bar{\nabla} H_{\rho}\right) \bar{\nabla} H_{\rho}\right) \\
& \left.\quad+<\mathbf{D}(\mathbf{v}) \mathbf{n}, \mathbf{n}>\tilde{\mathbf{V}}_{\mathbf{n}}\left(\bar{\nabla} H_{\rho}\right) \bar{\nabla} H_{\rho}\right\}-\mu\left(\mathcal{D}_{\mathbf{D}}(\mathbf{k}) \nabla \mathbf{v}\right)\left(\mathbf{n}+\tilde{\mathbf{V}}_{\mathbf{n}}\left(\bar{\nabla} H_{\rho}\right) \bar{\nabla} H_{\rho}\right) \\
& \quad-\mu<\left(\mathcal{D}_{\mathbf{D}}(\mathbf{k}) \nabla \mathbf{v}\right)\left(\mathbf{n}+\tilde{\mathbf{V}}_{\mathbf{n}}\left(\bar{\nabla} H_{\rho}\right) \bar{\nabla} H_{\rho}\right), \mathbf{n} \\
& \quad+\tilde{\mathbf{V}}_{\mathbf{n}}\left(\bar{\nabla} H_{\rho}\right) \bar{\nabla} H_{\rho}>\left(\mathbf{n}+\tilde{\mathbf{V}}_{\mathbf{n}}\left(\bar{\nabla} H_{\rho}\right) \bar{\nabla} H_{\rho}\right) .
\end{aligned}
$$

Finally, we derive the nonlinear term $h_{N}(\mathbf{u}, \rho)$ in (3.29). Recall that $\Gamma_{t}$ is represented by $x=(R+\rho) \mathbf{n}(y)+\xi(t)$ for $y \in S_{R}$, where $\mathbf{n}=y /|y| \in S_{1}$. Then, we have

$$
\frac{\partial x}{\partial p_{j}}=(R+\rho) \tau_{j}+\frac{\partial \rho}{\partial p_{j}} \mathbf{n}
$$


where $\tau_{j}=\frac{\partial \mathbf{n}}{\partial p_{j}}$, which forms a basis of the tangent space of $S_{1}$. Since $\tau_{j} \cdot \mathbf{n}=0$, the $(i, j)$ th component of the first fundamental form $G_{t}=\left(g_{t i j}\right)$ of $\Gamma_{t}$ is given by

$$
g_{t i j}=\frac{\partial x}{\partial p_{i}} \cdot \frac{\partial x}{\partial p_{j}}=(R+\rho)^{2} g_{i j}+\frac{\partial \rho}{\partial p_{i}} \frac{\partial \rho}{\partial p_{j}},
$$

where $g_{i j}=\tau_{i} \cdot \tau_{j}$ is the $(i, j)$ th element of the first fundamental form, $G$, of $S_{1}$, and so

$$
\begin{aligned}
G_{t} & =(R+\rho)^{2}\left(G+(R+\rho)^{-2} \nabla_{\Gamma}^{\prime} \rho \otimes \nabla_{\Gamma}^{\prime} \rho\right) \\
& =(R+\rho)^{2} G\left(\mathbf{I}+(R+\rho)^{-2}\left(G^{-1} \nabla_{\Gamma}^{\prime} \rho\right) \otimes \nabla_{\Gamma}^{\prime} \rho\right) .
\end{aligned}
$$

Since

$$
\operatorname{det}\left(\mathbf{I}+\mathbf{a}^{\prime} \otimes \mathbf{b}^{\prime}\right)=1+\mathbf{a}^{\prime} \cdot \mathbf{b}^{\prime}, \quad\left(\mathbf{I}+\mathbf{a}^{\prime} \otimes \mathbf{b}^{\prime}\right)^{-1}=\mathbf{I}-\frac{\mathbf{a}^{\prime} \otimes \mathbf{b}^{\prime}}{1+\mathbf{a}^{\prime} \cdot \mathbf{b}^{\prime}}
$$

for any $(N-1)$-vectors $\mathbf{a}^{\prime}$ and $\mathbf{b}^{\prime} \in \mathbb{R}^{N-1}$, we have

$$
\begin{aligned}
G_{t}^{-1} & =(R+\rho)^{-2}\left(\mathbf{I}-\frac{(R+\rho)^{-2}\left(G^{-1} \nabla_{\Gamma}^{\prime} \rho\right) \otimes \nabla_{\Gamma}^{\prime} \rho}{1+(R+\rho)^{-2}<G^{-1} \nabla_{\Gamma}^{\prime} \rho, \nabla_{\Gamma}^{\prime} \rho>}\right) G^{-1} \\
& =(R+\rho)^{-2} G^{-1}+O_{2} .
\end{aligned}
$$

Here and in the following, $\mathrm{O}_{2}$ denotes a symbol defined by setting

$$
O_{2}=a_{0} H_{\rho}^{2}+\sum_{j=1}^{N} b_{j} H_{\rho} \frac{\partial H_{\rho}}{\partial y_{j}}+\sum_{i, j=1}^{N} c_{i j} \frac{\partial H_{\rho}}{\partial y_{i}} \frac{\partial H_{\rho}}{\partial y_{j}}
$$

with some coefficients $a_{0}, b_{j}$ and $c_{i j}$ defined on $\overline{B_{R}}$ satisfying the estimate: $\left|\left(a_{0}, b_{j}, c_{i j}\right)(y, t)\right| \leq C$ and $\left|\nabla\left(a_{0}, b_{j}, c_{i j}\right)(y, t)\right| \leq C\left|\nabla^{2} H_{\rho}(y, t)\right|$ provided that $\left\|H_{\rho}\right\|_{L_{\infty}\left((0,2 \pi), H_{\infty}^{1}\left(B_{R}\right)\right)} \leq \delta$. In particular,

$$
g_{t}^{i j}=(R+\rho)^{-2} g^{i j}+O_{2},
$$

componentwise.

We next calculate the Christoffel symbols of $\Gamma_{t}$. Since

$$
\begin{aligned}
\tau_{t i} & =(R+\rho) \tau_{i}+\frac{\partial \rho}{\partial p_{i}} \mathbf{n}, \\
\tau_{t i j} & =(R+\rho) \tau_{i j}+\frac{\partial \rho}{\partial p_{j}} \tau_{i}+\frac{\partial \rho}{\partial p_{i}} \tau_{j}+\frac{\partial^{2} \rho}{\partial p_{i} \partial p_{j}} \mathbf{n},
\end{aligned}
$$

we have

$$
<\tau_{t i j}, \tau_{t \ell}>=(R+\rho)^{2}<\tau_{i j}, \tau_{\ell}>+(R+\rho)\left(\frac{\partial \rho}{\partial p_{\ell}} \ell_{i j}+g_{i \ell} \frac{\partial \rho}{\partial p_{j}}+g_{j \ell} \frac{\partial \rho}{\partial p_{i}}\right)
$$




$$
+\frac{\partial^{2} \rho}{\partial p_{i} \partial p_{j}} \frac{\partial \rho}{\partial p_{\ell}},
$$

where $\ell_{i j}=<\tau_{i j}, \mathbf{n}>$, and so

$$
\begin{aligned}
\Lambda_{t i j}^{k}= & g_{t}^{k \ell}<\tau_{t i j}, \tau_{t \ell}> \\
= & \left((R+\rho)^{-2} g^{k \ell}+O_{2}\right)\left((R+\rho)^{2}<\tau_{i j}, \tau_{\ell}>\right. \\
& \left.+(R+\rho)\left(\frac{\partial \rho}{\partial p_{\ell}} \ell_{i j}+g_{i \ell} \frac{\partial \rho}{\partial p_{j}}+g_{j \ell} \frac{\partial \rho}{\partial p_{i}}\right)+\frac{\partial^{2} \rho}{\partial p_{i} \partial p_{j}} \frac{\partial \rho}{\partial p_{\ell}}\right) \\
= & \Lambda_{i j}^{k}+(R+\rho)^{-1} g^{k \ell}\left(\frac{\partial \rho}{\partial p_{\ell}} \ell_{i j}+\delta_{i}^{k} \frac{\partial \rho}{\partial p_{j}}+\delta_{j}^{k} \frac{\partial \rho}{\partial p_{i}}\right) \\
& +\left((R+\rho)^{-2} g^{k \ell} \frac{\partial \rho}{\partial p_{\ell}}+O_{2}\right) \frac{\partial^{2} \rho}{\partial p_{i} \partial p_{j}}+O_{2} .
\end{aligned}
$$

Thus,

$$
\begin{aligned}
& \Delta_{\Gamma_{t}} f=g_{t}^{i j}\left(\partial_{i} \partial_{j} f-\Lambda_{t i j}^{k} \partial_{k} f\right) \\
& \quad=(R+\rho)^{-2} g^{i j}\left(\partial_{i} \partial_{j} f-\Lambda_{i j}^{k} \partial_{k} f\right)+\left(A^{k}\left(\nabla_{p}^{\prime} \rho, \nabla_{p}^{\prime 2} \rho\right) \partial_{k} f+O_{2} \otimes\left(\bar{\nabla}^{\prime 2} f\right)\right.
\end{aligned}
$$

where $\bar{\nabla}^{\prime 2} f$ is an $\left((N-1)^{2}+N\right)$-vector of the form: $\bar{\nabla}^{\prime 2} f=\left(\partial_{i} \partial_{j} f, \partial_{i} f, f \mid i, j=\right.$ $1, \ldots, N-1), \partial_{i}=\partial / \partial p_{i}, \nabla_{p}^{\prime 2}=\left(\partial_{i} \partial_{j} \rho \mid i, j=1, \ldots, N-1\right)$, and

$$
\begin{aligned}
A^{k}\left(\nabla_{p}^{\prime} \rho, \nabla_{p}^{\prime 2} \rho\right)= & -(R+\rho)^{-3} g^{i j} g^{k \ell}\left(\frac{\partial \rho}{\partial p_{\ell}} \ell_{i j}+\delta_{i}^{k} \frac{\partial \rho}{\partial p_{j}}+\delta_{j}^{k} \frac{\partial \rho}{\partial p_{i}}\right) \\
& -(R+\rho)^{-2}\left((R+\rho)^{-2} g^{i j} g^{k \ell} \frac{\partial \rho}{\partial p_{\ell}}+g^{i j} O_{2}\right) \frac{\partial^{2} \rho}{\partial p_{i} \partial p_{j}},
\end{aligned}
$$

and so

$$
\begin{aligned}
H\left(\Gamma_{t}\right) \mathbf{n}_{t}= & \Delta_{\Gamma_{t}}[(R+\rho) \mathbf{n}+\xi(t)] \\
= & (R+\rho)^{-2} g^{i j}\left(\partial_{i} \partial_{j}-\Lambda_{i j}^{k} \partial_{k}\right)((R+\rho) \mathbf{n})+\left(A^{k} \nabla_{p}^{2} \rho\right) \partial_{k}((R+\rho) \mathbf{n}) \\
& +O_{2} \otimes \bar{\nabla}^{\prime 2}((R+\rho) \mathbf{n}) \\
= & (R+\rho)^{-1} g^{i j}\left(\partial_{i} \partial_{j} \mathbf{n}-\Lambda_{i j}^{k} \partial_{k} \mathbf{n}\right)+(R+\rho)^{-2} g^{i j}\left(\partial_{i} \rho \partial_{j} \mathbf{n}+\partial_{j} \rho \partial_{i} \mathbf{n}\right) \\
+ & (R+\rho)^{-2} g^{i j}\left(\partial_{i} \partial_{j} \rho-\Lambda_{i j}^{k} \partial_{k} \rho\right) \mathbf{n}+A^{k}\left(\nabla_{p}^{\prime} \rho, \nabla_{p}^{\prime 2} \rho\right)\left(\partial_{k} \rho\right) \mathbf{n} \\
+ & A^{k}\left(\nabla_{p}^{\prime} \rho, \nabla_{p}^{\prime 2} \rho\right)(R+\rho) \partial_{k} \mathbf{n}+O_{2} \otimes \bar{\nabla}^{\prime 2}(R+\rho)
\end{aligned}
$$

Combining this formula with (3.21), using $<\partial_{i} \mathbf{n}, \mathbf{n}>=0,<\mathbf{n}, \tau_{\ell}>=0, \Delta_{S_{1}} \mathbf{n}=$ $-(N-1) \mathbf{n}$, and (3.15) gives

$$
\begin{aligned}
& <H\left(\Gamma_{t}\right) \mathbf{n}_{t}, \mathbf{n}_{t}> \\
& \quad=-(R+\rho)^{-1}(N-1)+(R+\rho)^{-2} \Delta_{S_{1}} \rho+\left(O_{1}+O_{2}\right) \otimes \nabla_{p}^{2} \rho+O_{2},
\end{aligned}
$$


where $O_{1}$ denotes a symbol defined by setting

$$
O_{1}=a_{0}^{\prime} H_{\rho}+\sum_{j=1}^{N} b_{j}^{\prime} \frac{\partial H_{\rho}}{\partial y_{j}}
$$

with some coefficients $a_{0}^{\prime}$ and $b_{j}^{\prime}$ defined on $\overline{B_{R}}$ satisfying the estimate: $\left|\left(a_{0}^{\prime}, b_{j}^{\prime}\right)(y, t)\right| \leq$ $C$ and $\left|\nabla\left(a_{0}^{\prime}, b_{j}^{\prime}\right)(y, t)\right| \leq C\left|\nabla^{2} H_{\rho}(y, t)\right|$ provided that $\left\|H_{\rho}\right\|_{L_{\infty}\left((0,2 \pi), H_{\infty}^{1}\left(B_{R}\right)\right)} \leq \delta$. Since

$$
\begin{aligned}
(R+\rho)^{-1} & =R^{-1}-\rho R^{-2}+O\left(\rho^{2}\right), \\
(R+\rho)^{-2} \Delta_{S_{1}} \rho & =R^{-2} \Delta_{S_{1}} \rho+2 R^{-3} \rho \Delta_{S_{1}} \rho+O_{2} \otimes \nabla_{p}^{2} \rho,
\end{aligned}
$$

we have

$$
<H\left(\Gamma_{t}\right) \mathbf{n}_{t}, \mathbf{n}_{t}>=-\frac{N-1}{R}+\mathcal{B} \rho+\left(O_{1}+O_{2}\right) \otimes \nabla_{p}^{2} \rho+O_{2} .
$$

Setting $p_{0}=-(N-1) / R$, from (3.27) we have

$$
<\mu \mathbf{D}(\mathbf{v}) \mathbf{n}, \mathbf{n}>-\mathfrak{q}-\sigma \mathcal{B} \rho=h_{N}(\mathbf{v}, \rho)
$$

on $S_{R} \times(0,2 \pi)$. Here, in view of (3.3) and (3.33), we have defined $h_{N}(\mathbf{v}, \rho)$ by letting

$$
h_{N}(\mathbf{v}, \rho)=\mathbf{V}_{h, N}\left(\bar{\nabla} H_{\rho}\right) \bar{\nabla} H_{\rho} \otimes \nabla \mathbf{v}+\sigma \tilde{\mathbf{V}}_{\Gamma}^{\prime}\left(\bar{\nabla} H_{\rho}\right) \bar{\nabla} H_{\rho} \otimes \bar{\nabla}^{2} H_{\rho},
$$

where $\mathbf{V}_{h, N}(\mathbf{k})$ and $\tilde{\mathbf{V}}_{\Gamma}^{\prime}(\mathbf{k})$ are functions defined on $\overline{B_{R}} \times\{\mathbf{k}|| \mathbf{k} \mid<\delta\}$ possessing the estimate:

$$
\begin{aligned}
& \sup _{|\mathbf{k}|<\delta}\left\|\left(\mathbf{V}_{h, N}(\cdot, \mathbf{k}), \partial_{\mathbf{k}} \mathbf{V}_{h, N}(\cdot, \mathbf{k})\right)\right\|_{H_{\infty}^{1}\left(B_{R}\right)} \leq C, \\
& \sup _{|\mathbf{k}|<\delta}\left\|\left(\tilde{\mathbf{V}}_{\Gamma}^{\prime}(\cdot, \mathbf{k}), \partial_{\mathbf{k}} \tilde{\mathbf{V}}_{\Gamma}^{\prime}(\cdot, \mathbf{k})\right)\right\|_{H_{\infty}^{1}\left(B_{R}\right)} \leq C
\end{aligned}
$$

for some constant $C$.

\subsection{Two-phase problem case}

Let $\Omega_{+}=B_{R}$ and $\Omega_{-}=\Omega \backslash\left(B_{R} \cup S_{R}\right)$. In the two-phase case, we let

$$
\Psi_{+}(y, t)=R^{-1} H_{\rho}(y, t) y+\xi(t), \quad \Psi_{-}(y, t)=\varphi(y)\left(R^{-1} H_{\rho}(y, t) y+\xi(t)\right) .
$$

Let $J_{ \pm}(t)$ be the Jacobian of the map: $x=y+\Psi_{ \pm}(y, t)$ for $y \in \Omega_{ \pm}$, which are defined by setting

$$
\begin{cases}J_{+}(t)=\operatorname{det}\left(I+R^{-1} \nabla_{y}\left(H_{\rho}(y, t) y\right)\right) & \text { for } y \in \Omega_{+}, \\ J_{-}(t)=\operatorname{det}\left(I+\nabla_{y}\left(\varphi(y)\left(R^{-1}\left(H_{\rho}(y, t) y+\xi(t)\right)\right)\right.\right. & \text { for } y \in \Omega_{-} .\end{cases}
$$


Notice that

$$
\xi(t)=\int_{0}^{t} \int_{B_{R}} \mathbf{v}_{+}(y, s) J_{+}(s) \mathrm{d} y \mathrm{~d} s+c
$$

where $c$ is the unique constant for which the following equality holds:

$$
\int_{0}^{2 \pi} \xi(t)=0
$$

We assume that

$$
\sup _{t \in(0,2 \pi)}\left\|H_{\rho}(\cdot, t)\right\|_{H_{\infty}^{1}\left(\Omega_{ \pm}\right)} \leq \delta, \quad \sup _{t \in(0,2 \pi)}|\xi(t)| \leq \delta
$$

with suitably small constant $\delta>0$. Since

$$
|\xi(t)| \leq C \sup _{t \in(0,2 \pi)}\|\mathbf{v}(\cdot, t)\|_{L_{q}\left(B_{R}\right)} \sup _{t \in(0,2 \pi)}\left|J_{+}(t)\right|\left|B_{R}\right|,
$$

there exists a constant $\delta_{1}>0$ such that if

$$
\sup _{t \in(0,2 \pi)}\left\|\mathbf{v}_{+}(\cdot, t)\right\|_{L_{q}\left(B_{R}\right)} \leq \delta_{1}
$$

then the condition for $\xi(t)$ in (3.36) holds. Thus, in the proof of Theorem 5, we assume that the conditions (3.36) and (3.37) hold.

Set $J_{0 \pm}(t)=J_{ \pm}(t)-1$. By the chain rule, we have

$$
\nabla_{x}=\left(\mathbf{I}+\mathbf{V}_{ \pm 0}\left(\mathbf{k}_{ \pm}\right)\right) \nabla_{y}, \quad \frac{\partial}{\partial x_{i}}+\sum_{j=1}^{N} V_{ \pm 0 i j}\left(\mathbf{k}_{ \pm}\right) \frac{\partial}{\partial y_{j}}
$$

where $\mathbf{V}_{ \pm 0}\left(\mathbf{k}_{ \pm}\right)$is given by

$$
\mathbf{V}_{ \pm 0}\left(\mathbf{k}_{ \pm}\right)= \begin{cases}\left(\mathbf{I}+\nabla_{y}\left(R^{-1} H_{\rho}(y, t) y\right)^{-1}-\mathbf{I}\right. & \text { for } y \in \Omega_{+} \\ \left(\mathbf{I}+\nabla_{y} \Psi_{-, \rho}(y, t)\right)^{-1}-\mathbf{I} & \text { for } y \in \Omega_{-} .\end{cases}
$$

Here and in the following, $\mathbf{k}_{+}$and $\mathbf{k}_{-}$denote the variables corresponding to $\left(H_{\rho}, \nabla H_{\rho}\right)$ and $\left(\Psi_{-, \rho}, \nabla \Psi_{-, \rho}\right)$.

Employing the same argument as for obtaining the formulas in (3.6), we have

$$
\begin{aligned}
& g_{ \pm}(\mathbf{v}, \rho)=-\left(J_{0 \pm}\left(\mathbf{k}_{ \pm}\right) \operatorname{div} \mathbf{v}_{ \pm}+\left(1+J_{0 \pm}\left(\mathbf{k}_{ \pm}\right)\right) \mathbf{V}_{0 \pm}\left(\mathbf{k}_{ \pm}\right): \nabla \mathbf{v}_{ \pm}\right) \\
& \mathbf{g}_{ \pm}(\mathbf{v}, \rho)=-\left(1+J_{0 \pm}\left(\mathbf{k}_{ \pm}\right)\right)^{\top} \mathbf{V}_{0 \pm}\left(\mathbf{k}_{ \pm}\right) \mathbf{v}_{ \pm} .
\end{aligned}
$$

And also, from (3.13) we have

$$
\begin{gathered}
\mathbf{G}_{ \pm}(y, t)=\left(\mathbf{I}+\nabla \Psi_{ \pm}(y, t)\right) \mathbf{f}\left(y+\Psi_{ \pm}(y, t), t\right), \\
\mathbf{F}_{ \pm}(\mathbf{v}, \rho)={ }^{\top}\left(F_{1 \pm}(\mathbf{v}, \rho), \ldots, F_{N \pm}(\mathbf{v}, \rho)\right)
\end{gathered}
$$


with

$$
\begin{aligned}
F_{i \pm}(\mathbf{v}, \rho)= & -\sum_{j, k=1}^{N}\left(v_{ \pm j}-\frac{\partial \Psi_{ \pm j}}{\partial t}\right)\left(\delta_{j k}+V_{0 j k}\left(\mathbf{k}_{ \pm}\right)\right) \frac{\partial v_{ \pm i}}{\partial y_{k}} \\
& -\sum_{\ell=1}^{N} \partial_{i} \Psi_{ \pm \ell}\left(\frac{\partial v_{ \pm \ell}}{\partial t}+\sum_{j, k=1}^{N}\left(v_{ \pm j}-\frac{\partial \Psi_{ \pm j}}{\partial t}\right)\left(\delta_{j k}+V_{ \pm 0 j k}\left(\mathbf{k}_{ \pm}\right)\right) \frac{\partial v_{ \pm \ell}}{\partial y_{k}}\right) \\
& +\mu\left(\sum_{j=1}^{N} \frac{\partial}{\partial y_{j}}\left(\mathcal{D}_{\mathbf{D}}\left(\mathbf{k}_{ \pm}\right) \nabla \mathbf{v}_{ \pm}\right)_{i j}+\sum_{j, k=1}^{N} V_{0 j k}\left(\mathbf{k}_{ \pm}\right) \frac{\partial}{\partial y_{k}}\left(\mathbf{D}\left(\mathbf{v}_{ \pm}\right)_{i j}+\left(\mathcal{D}_{\mathbf{D}}\left(\mathbf{k}_{ \pm}\right) \nabla \mathbf{v}_{ \pm}\right)_{i j}\right)\right. \\
& \left.+\sum_{j, k, \ell=1}^{N} \partial_{i} \Psi_{ \pm \ell}\left(\delta_{j k}+V_{ \pm 0 j k}(\mathbf{k})\right) \frac{\partial}{\partial y_{k}}\left(\mathbf{D}\left(\mathbf{v}_{ \pm}\right)_{\ell j}+\left(\mathcal{D}_{\mathbf{D}}\left(\mathbf{k}_{ \pm}\right) \nabla \mathbf{v}_{ \pm}\right)_{\ell j}\right)\right) .
\end{aligned}
$$

Here and in the following, we have set $\Psi_{ \pm}(y, t)={ }^{\top}\left(\Psi_{ \pm 1}(y, t), \ldots, \Psi_{ \pm N}(y, t)\right)$, $\mathbf{v}_{ \pm}={ }^{\top}\left(v_{ \pm 1}, \ldots, v_{ \pm N}\right)$, and

$$
\left(\mathcal{D}_{\mathbf{D}}\left(\mathbf{k}_{ \pm}\right) \nabla \mathbf{v}_{ \pm}\right)_{i j}=\sum_{k=1}^{N}\left(V_{ \pm 0 j k}\left(\mathbf{k}_{ \pm}\right) \frac{\partial v_{ \pm i}}{\partial y_{k}}+V_{ \pm 0 i k}\left(\mathbf{k}_{ \pm}\right) \frac{\partial v_{ \pm j}}{\partial y_{k}}\right) .
$$

To define the right hand side of the transmission condition, we use (3.31) and (3.34). We first introduce a symbol $((\cdot))$. For $f_{ \pm}$, let $\left[f_{ \pm}\right]$be a suitable extension of $f_{ \pm}$to $\Omega_{\mp}$ such that

$$
\left\|\left[f_{ \pm}\right]\right\|_{H_{q}^{k}\left(\Omega_{\mp}\right)} \leq C_{k}\left\|f_{ \pm}\right\|_{H_{q}^{k}\left(\Omega_{ \pm}\right)}, \quad\left\|\partial_{t}\left[f_{ \pm}\right]\right\|_{H_{q}^{k}\left(\Omega_{\mp}\right)} \leq C_{k}\left\|\partial_{t} f_{ \pm}\right\|_{H_{q}^{k}\left(\Omega_{ \pm}\right)}
$$

with some constant $C_{k}$. Here, if the right-hand side is finite, then $\left[f_{ \pm}\right]$and $\partial_{t}\left[f_{ \pm}\right]$exist and the estimates above hold. In particular, we set $H_{q}^{0}\left(\Omega_{ \pm}\right)=L_{q}\left(\Omega_{ \pm}\right)$. We set

$$
\operatorname{ex}\left[f_{ \pm}\right](y, t)= \begin{cases}f_{ \pm}(y, t) & \text { for } y \in \Omega_{ \pm}, \\ {\left[f_{ \pm}\right](y, t)} & \text { for } y \in \Omega_{\mp} .\end{cases}
$$

And then, $((f))$ is defined by setting

$$
((f))=\operatorname{ex}\left[f_{+}\right]-\operatorname{ex}\left[f_{-}\right] .
$$

Using this symbol, we can proceed as for the derivation of (3.31) and (3.34) and define $\tilde{\mathbf{h}}^{\prime}(\mathbf{v}, \rho)$ and $\tilde{h}_{N}(\mathbf{v}, \rho)$ by setting

$$
\begin{aligned}
& \tilde{\mathbf{h}}^{\prime}(\mathbf{v}, \rho)=-\mu((\mathbf{D}(\mathbf{v}))) \tilde{\mathbf{V}}_{\mathbf{n}}\left(\bar{\nabla} H_{\rho}\right) \bar{\nabla} H_{\rho} \\
& \quad+\mu\{<((\mathbf{D}(\mathbf{v})))) \tilde{\mathbf{V}}_{\mathbf{n}}\left(\bar{\nabla} H_{\rho}\right) \bar{\nabla} H_{\rho}, \mathbf{n}+\tilde{\mathbf{V}}_{\mathbf{n}}\left(\bar{\nabla} H_{\rho}\right) \bar{\nabla} H_{\rho}>\left(\mathbf{n}+\tilde{\mathbf{V}}_{\mathbf{n}}\left(\bar{\nabla} H_{\rho}\right) \bar{\nabla} H_{\rho}\right) \\
& \quad+<((\mathbf{D}(\mathbf{v}))) \mathbf{n}, \tilde{\mathbf{V}}_{\mathbf{n}}\left(\bar{\nabla} H_{\rho}\right) \bar{\nabla} H_{\rho}>\left(\mathbf{n}+\tilde{\mathbf{V}}_{\mathbf{n}}\left(\bar{\nabla} H_{\rho}\right) \bar{\nabla} H_{\rho}\right) \\
& \left.\quad+<((\mathbf{D}(\mathbf{v}))) \mathbf{n}, \mathbf{n}>\tilde{\mathbf{V}}_{\mathbf{n}}\left(\bar{\nabla} H_{\rho}\right) \bar{\nabla} H_{\rho}\right\}-\mu\left(\left(\mathcal{D}_{\mathbf{D}}(\mathbf{k}) \nabla \mathbf{v}\right)\right)\left(\mathbf{n}+\tilde{\mathbf{V}}_{\mathbf{n}}\left(\bar{\nabla} H_{\rho}\right) \bar{\nabla} H_{\rho}\right) \\
& \quad-\mu<\left(\left(\mathcal{D}_{\mathbf{D}}(\mathbf{k}) \nabla \mathbf{v}\right)\right)\left(\mathbf{n}+\tilde{\mathbf{V}}_{\mathbf{n}}\left(\bar{\nabla} H_{\rho}\right) \bar{\nabla} H_{\rho}\right), \mathbf{n}+\tilde{\mathbf{V}}_{\mathbf{n}}\left(\bar{\nabla} H_{\rho}\right) \bar{\nabla} H_{\rho}>\left(\mathbf{n}+\tilde{\mathbf{V}}_{\mathbf{n}}\left(\bar{\nabla} H_{\rho}\right) \bar{\nabla} H_{\rho}\right) \\
& \tilde{h}_{N}(\mathbf{v}, \rho)=\mathbf{V}_{h, N}\left(\bar{\nabla} H_{\rho}\right) \bar{\nabla} H_{\rho} \otimes((\nabla \mathbf{v}))+\sigma \tilde{\mathbf{V}}_{\Gamma}^{\prime}\left(\bar{\nabla} H_{\rho}\right) \bar{\nabla} H_{\rho} \otimes \bar{\nabla}^{2} H_{\rho} .
\end{aligned}
$$

And then, we set $\tilde{\mathbf{h}}(\mathbf{v}, \rho)=\left(\tilde{\mathbf{h}}^{\prime}(\mathbf{v}, \rho), \tilde{h}_{N}(\mathbf{v}, \rho)\right)$. 


\section{On periodic solutions of the linearized equations}

In this section, we shall prove the $L_{p}-L_{q}$ maximal regularity of $2 \pi$-periodic solutions of the linearized equations.

\subsection{On linearized problem of one-phase problem}

In this subsection, we consider the $L_{p}-L_{q}$ maximal regularity of periodic solutions to linearized equations:

$$
\begin{aligned}
\partial_{t} \mathbf{u}+\mathcal{L} \mathbf{u}_{S}-\operatorname{Div}(\mu \mathbf{D}(\mathbf{u})-\mathfrak{p} \mathbf{I})=\mathbf{F} & \text { in } B_{R} \times(0,2 \pi), \\
\operatorname{div} \mathbf{u}=G=\operatorname{div} \mathbf{G} & \text { in } B_{R} \times(0,2 \pi), \\
\partial_{t} \rho+\mathcal{M} \rho-(\mathcal{A} \mathbf{u}) \cdot \mathbf{n}=D & \text { on } S_{R} \times(0,2 \pi), \\
(\mu \mathbf{D}(\mathbf{u})-\mathfrak{p} \mathbf{I}) \mathbf{n}-\left(\mathcal{B}_{R} \rho\right) \mathbf{n}=\mathbf{H} & \text { on } S_{R} \times(0,2 \pi),
\end{aligned}
$$

where $\mathcal{L}, \mathcal{M}$, and $\mathcal{A}$ are the linear operators defined in (2.17). We shall prove the unique existence theorem of $2 \pi$-periodic solutions of equations (4.1). Our main result is this section is stated as follows.

Theorem 6. Let $1<p, q<\infty$. Then, for any $\mathbf{F}, D, G, \mathbf{G}$ and $\mathbf{H}$ with

$$
\begin{aligned}
& \mathbf{F} \in L_{p, \operatorname{per}}\left((0,2 \pi), L_{q}\left(B_{R}\right)^{N}\right), \quad D \in L_{p, \operatorname{per}}\left((0,2 \pi), W_{q}^{2-1 / q}\left(S_{R}\right)\right) \\
& G \in L_{p, \operatorname{per}}\left((0,2 \pi), H_{q}^{1}\left(B_{R}\right)\right) \cap H_{p, \text { per }}^{1 / 2}\left((0,2 \pi), L_{q}\left(B_{R}\right)\right), \quad \mathbf{G} \in H_{p, \operatorname{per}}^{1}\left((0,2 \pi) . L_{q}\left(B_{R}\right)^{N}\right), \\
& \mathbf{H} \in L_{p, \operatorname{per}}\left((0,2 \pi), H_{q}^{1}\left(B_{R}\right)^{N}\right) \cap H_{p, \text { per }}^{1 / 2}\left((0,2 \pi), L_{q}\left(B_{R}\right)^{N}\right),
\end{aligned}
$$

problem (4.1) admits unique solutions $\mathbf{u}, \mathfrak{p}$ and $\rho$ with

$$
\begin{aligned}
& \mathbf{u} \in L_{p, \text { per }}\left((0,2 \pi), H_{q}^{2}\left(B_{R}\right)^{N}\right) \cap H_{p, \text { per }}^{1}\left((0,2 \pi), L_{q}\left(B_{R}\right)^{N}\right), \\
& \mathfrak{p} \in L_{p, \operatorname{per}}\left((0,2 \pi), H_{q}^{1}\left(B_{R}\right)\right), \\
& \rho \in L_{p, \operatorname{per}}\left((0,2 \pi), W_{q}^{3-1 / q}\left(S_{R}\right)\right) \cap H_{p, \text { per }}^{1}\left((0,2 \pi), W_{q}^{2-1 / q}\left(S_{R}\right)\right)
\end{aligned}
$$

possessing the estimate:

$$
\begin{aligned}
\|\mathbf{u}\|_{L_{p}\left((0,2 \pi), H_{q}^{2}\left(B_{R}\right)\right)}+\left\|\partial_{t} \mathbf{u}\right\|_{L_{p}\left((0,2 \pi), L_{q}\left(B_{R}\right)\right)}+\|\nabla \mathfrak{p}\|_{L_{p}\left((0,2 \pi), L_{q}\left(B_{R}\right)\right)} & \quad\|\rho\|_{L_{p}\left((0,2 \pi), W_{q}^{3-1 / q}\left(S_{R}\right)\right)}+\left\|\partial_{t} \rho\right\|_{L_{p}\left((0,2 \pi), W_{q}^{2-1 / q}\left(S_{R}\right)\right)} \\
& \quad+\|\left\{\|\mathbf{F}\|_{L_{p}\left((0,2 \pi), L_{q}\left(B_{R}\right)\right)}+\|D\|_{L_{p}\left((0,2 \pi), W_{q}^{2-1 / q}\left(S_{R}\right)\right)}+\left\|\partial_{t} \mathbf{G}\right\|_{L_{p}\left((0,2 \pi), L_{q}\left(B_{R}\right)\right)}\right. \\
& \left.+\|(G, \mathbf{H})\|_{L_{p}\left((0,2 \pi), H_{q}^{1}\left(B_{R}\right)\right)}+\|(G, \mathbf{H})\|_{H_{p}^{1 / 2}\left((0,2 \pi), L_{q}\left(B_{R}\right)\right)}\right\}
\end{aligned}
$$

for some constant $C>0$.

To prove Theorem 6, our approach is to use the $\mathcal{R}$-solver, Weis' operator-valued Fourier multiplier theorem [22] and a transference theorem, which is created in Eiter, Kyed and Shibata [2]. To introduce the notion of $\mathcal{R}$-solver, we introduce the $\mathcal{R}$ boundedness of operator families. 
Definition 7. Let $X$ and $Y$ be two Banach spaces. A family of operators $\mathcal{T} \subset \mathcal{L}(X, Y)$ is called $\mathcal{R}$-bounded on $\mathcal{L}(X, Y)$, if there exist a constant $C>0$ and $p \in[1, \infty)$ such that for each $n \in \mathbb{N},\left\{T_{j}\right\}_{j=1}^{n} \in \mathcal{T}^{n}$, and $\left\{f_{j}\right\}_{j=1}^{n} \in X^{n}$, we have

$$
\left\|\sum_{k=1}^{n} r_{k} T_{k} f_{k}\right\|_{L_{p}((0,1), Y)} \leq C\left\|\sum_{k=1}^{n} r_{k} f_{k}\right\|_{L_{p}((0,1), X)} .
$$

Here, the Rademacher functions $r_{k}, k \in \mathbb{N}$, are given by $r_{k}:[0,1] \rightarrow\{-1,1\}$, $t \mapsto \operatorname{sign}\left(\sin 2^{k} \pi t\right)$. The smallest such $C$ is called $\mathcal{R}$-bound of $\mathcal{T}$ on $\mathcal{L}(X, Y)$, which is denoted by $\mathcal{R}_{\mathcal{L}(X, Y)} \mathcal{T}$.

We quote Weis' operator-valued Fourier multiplier theorem and the transference theorem for operator-valued Fourier multipliers.

Theorem 8. [Weis] Let $X$ and $Y$ be two UMD Banach spaces. Let $m \in C^{1}$ $(\mathbb{R} \backslash\{0\}, \mathcal{L}(X, Y))$ satisfies the multiplier condition:

$$
\mathcal{R}_{\mathcal{L}(X, Y)}\left\{\left(\tau \partial_{\tau}\right)^{\ell} m(\tau) \mid \tau \in \mathbb{R} \backslash\{0\}\right\} \leq r_{b}
$$

for $\ell=0,1$ with some constant $r_{b}$. Let $T_{m}$ be a multiplier defined by $T_{m}[f]=$ $\mathcal{F}^{-1}[m \mathcal{F}[f]]$. Then, $T_{m} \in \mathcal{L}\left(L_{p}(\mathbb{R}, X), L_{p}(\mathbb{R}, Y)\right)$ with

$$
\left\|T_{m}[f]\right\|_{L_{p}(\mathbb{R}, Y)} \leq C_{p} r_{b}\|f\|_{L_{p}(\mathbb{R}, X)}
$$

for any $p \in(1, \infty)$ with some constant $C_{p}$ depending on $p$ but independent of $r_{b}$.

The transference theorem for operator-valued Fourier multipliers obtained in [2] is stated as follows.

Theorem 9. Let $X$ and $Y$ be two Banach spaces and $p \in(1, \infty)$. Assume that $Y$ is reflexive. Let

$$
m \in L_{\infty}(\mathbb{R}, \mathcal{L}(X, Y)) \cap C(\mathbb{R}, \mathcal{L}(X, Y)),
$$

and let $\left.m\right|_{\mathbb{T}}$ denote the restriction of $m$ on $\mathbb{T}$. We define multipliers on $\mathbb{R}$ and $\mathbb{T}$ associated with $m$ by setting

$$
T_{m, \mathbb{R}}[f](t)=\mathcal{F}^{-1}[m \mathcal{F}[f]], \quad T_{m, \mathbb{T}}[f]=\mathcal{F}_{\mathbb{T}}^{-1}\left[\left.m\right|_{\mathbb{T}} \mathcal{F}_{\mathbb{T}}[f]\right] .
$$

If $T_{m, \mathbb{R}} \in \mathcal{L}\left(L_{p}(\mathbb{R}, X), L_{p}(\mathbb{R}, Y)\right)$ possessing the estimate:

$$
\left\|T_{m, \mathbb{R}}[f]\right\|_{L_{p}(\mathbb{R}, Y)} \leq M\|f\|_{L_{p}(\mathbb{R}, X)}
$$

for any $f \in L_{p}(\mathbb{R}, X)$ with some constant $M$, then $T_{m, \mathbb{T}} \in \mathcal{L}\left(L_{p}(\mathbb{T}, X), L_{p}(\mathbb{T}, Y)\right)$ and

$$
\left\|T_{m, \mathbb{T}}[f]\right\|_{L_{p}(\mathbb{T}, Y)} \leq C_{p} M\|f\|_{L_{p}(\mathbb{T}, X)}
$$

for any $f \in L_{p}(\mathbb{T}, X)$ with some constant $C_{p}$ depending solely on $p$ and independent of $M$. 
Remark 10. In the usual scalar-valued multiplier case, the transference theorem was proved by de Leeuw [1], and so this theorem is an extension to the operator-valued case.

We now consider the $\mathcal{R}$-solver of the generalized resolvent problem:

$$
\begin{aligned}
i k \mathbf{v}-\operatorname{Div}(\mu \mathbf{D}(\mathbf{v})-\mathfrak{q} \mathbf{I})=\mathbf{f} & \text { in } B_{R}, \\
\operatorname{div} \mathbf{v}=g=\operatorname{div} \mathbf{g} & \text { in } B_{R}, \\
i k \eta+\mathcal{M} \eta-(\mathcal{A} \mathbf{v}) \cdot \mathbf{n}=d & \text { on } S_{R}, \\
(\mu \mathbf{D}(\mathbf{v})-\mathfrak{q} \mathbf{I}) \mathbf{n}-\left(\mathcal{B}_{R} \eta\right) \mathbf{n}=\mathbf{h} & \text { on } S_{R}
\end{aligned}
$$

for $k \in \mathbb{R}$. From Theorem 4.8 in Shibata [18] (cf. also Shibata [15,16]) we know the following theorem concerned with the existence of an $\mathcal{R}$-solver of problem (4.1).

Theorem 11. Let $1<q<\infty$ and let $\mathbb{R}_{k_{0}}=\mathbb{R} \backslash\left(-k_{0}, k_{0}\right)$. Let

$$
\begin{aligned}
& X_{q}\left(B_{R}\right)=\left\{(\mathbf{f}, d, \mathbf{h}, g, \mathbf{g}) \mid \mathbf{f} \in L_{q}\left(B_{R}\right)^{N}, d \in W_{q}^{2-1 / q}\left(S_{R}\right),\right. \\
&\left.\mathbf{h} \in H_{q}^{1}\left(B_{R}\right)^{N}, g \in H_{q}^{1}\left(B_{R}\right), \mathbf{g} \in L_{q}\left(B_{R}\right)^{N}\right\}, \\
& \mathcal{X}_{q}\left(B_{R}\right)=\left\{F=\left(F_{1}, F_{2}, \ldots, F_{7}\right) \mid F_{1}, F_{3}, F_{7} \in L_{q}\left(B_{R}\right)^{N},\right. \\
& F_{2} \in W_{q}^{2-1 / q}\left(S_{R}\right), F_{4} \in H_{q}^{1}\left(B_{R}\right)^{N}, \\
&\left.F_{5} \in L_{q}\left(B_{R}\right), F_{6} \in H_{q}^{1}\left(B_{R}\right)\right\} .
\end{aligned}
$$

Then, there exist a constant $k_{0}>0$ and operator families $\mathcal{A}(i k), \mathcal{P}(i k)$, and $\mathcal{H}(i k)$ with

$$
\begin{aligned}
& \mathcal{A}(i k) \in C^{1}\left(\mathbb{R}_{k_{0}}, \mathcal{L}\left(\mathcal{X}_{q}\left(B_{R}\right), H_{q}^{2}\left(B_{R}\right)^{N}\right)\right), \\
& \mathcal{P}(i k) \in C^{1}\left(\mathbb{R}_{k_{0}}, \mathcal{L}\left(\mathcal{X}_{q}\left(B_{R}\right), H_{q}^{1}\left(B_{R}\right)\right)\right), \\
& \mathcal{H}(i k) \in C^{1}\left(\mathbb{R}_{k_{0}}, \mathcal{L}\left(\mathcal{X}_{q}\left(B_{R}\right), W_{q}^{3-1 / q}\left(S_{R}\right)\right)\right)
\end{aligned}
$$

such that for any $(\mathbf{f}, d, \mathbf{h}, g, \mathbf{g})$ and $k \in \mathbb{R}_{k_{0}}, \mathbf{v}=\mathcal{A}(i k) \mathcal{F}_{k}, \mathfrak{q}=\mathcal{P}(i k) \mathcal{F}_{k}$ and $\eta=\mathcal{H}\left(\right.$ ik) $\mathcal{F}_{k}$, where

$$
\mathcal{F}_{k}=\left(\mathbf{f}, d,(i k)^{1 / 2} \mathbf{h}, \mathbf{h},(i k)^{1 / 2} g, g, i k \mathbf{g}\right),
$$

are unique solutions of equations (4.3), and

$$
\begin{array}{r}
\mathcal{R}_{\mathcal{L}\left(\mathcal{X}_{q}\left(B_{R}\right), H_{q}^{2-m}\left(B_{R}\right)^{N}\right)}\left(\left\{\left(k \partial_{k}\right)^{\ell}\left((i k)^{m / 2} \mathcal{A}(i k)\right) \mid k \in \mathbb{R}_{k_{0}}\right\}\right) \leq r_{b}, \\
\mathcal{R}_{\mathcal{L}\left(\mathcal{X}_{q}\left(B_{R}\right), L_{q}\left(B_{R}\right)^{N}\right)}\left(\left\{\left(k \partial_{k}\right)^{\ell} \nabla \mathcal{P}(i k) \mid k \in \mathbb{R}_{k_{0}}\right\}\right) \leq r_{b}, \\
\mathcal{R}_{\mathcal{L}\left(\mathcal{X}_{q}\left(B_{R}\right), W_{q}^{3-n-1 / q}\left(S_{R}\right)\right)}\left(\left\{\left(k \partial_{k}\right)^{\ell}\left((i k)^{n} \mathcal{H}(i k)\right) \mid k \in \mathbb{R}_{k_{0}}\right\}\right) \leq r_{b}
\end{array}
$$

for $\ell=0,1, m=0,1,2$ and $n=0,1$ with some constant $r_{b}$. 
Remark 12. (1) Here and in the following, for $\theta \in(0,1)$ we set

$$
(i k)^{\theta}= \begin{cases}\mathrm{e}^{i \pi \theta / 2}|k|^{\theta} & \text { for } k>0, \\ \mathrm{e}^{-i \pi \theta / 2}|k|^{\theta} & \text { for } k<0 .\end{cases}
$$

(2) The functions $F_{1}, F_{2}, F_{3}, F_{4}, F_{5}, F_{6}$, and $F_{7}$ are variables corresponding to $\mathbf{f}$, $d,(i k)^{1 / 2} \mathbf{h}, \mathbf{h},(i k)^{1 / 2} g, g$, and $i k \mathbf{g}$, respectively.

(3) We define the norm $\|\cdot\|_{\mathcal{X}_{q}\left(B_{R}\right)}$ by setting

$$
\begin{aligned}
& \left\|\left(F_{1}, \ldots, F_{7}\right)\right\|_{\mathcal{X}_{q}\left(B_{R}\right)}=\left\|\left(F_{1}, F_{3}, F_{5}, F_{7}\right)\right\|_{L_{q}\left(B_{R}\right)} \\
& +\left\|F_{2}\right\|_{W_{q}^{2-1 / q}\left(S_{R}\right)}+\left\|\left(F_{4}, F_{6}\right)\right\|_{H_{q}^{1}\left(B_{R}\right)} .
\end{aligned}
$$

Let $\varphi(i k)$ be a function in $C^{\infty}(\mathbb{R})$ which equals one for $k \in \mathbb{R}_{k_{0}+2}$ and zero for $k \notin \mathbb{R}_{k_{0}+1}$, and let $\psi(i k)$ be a function in $C^{\infty}(\mathbb{R})$ which equals one for $k \in \mathbb{R}_{k_{0}+4}$ and zero for $k \notin \mathbb{R}_{k_{0}+3}$. Notice that $\varphi(i k) \psi(i k)=\varphi(i k)$. Let $\mathcal{A}(i k), \mathcal{P}(i k)$ and $\mathcal{H}(i k)$ be the $\mathcal{R}$-solvers given in Theorem 11 . Then, we have

$$
\begin{array}{r}
\mathcal{R}_{\mathcal{L}\left(\mathcal{X}_{q}\left(B_{R}\right), H_{q}^{2-m}\left(B_{R}\right)^{N}\right)}\left(\left\{\left(k \partial_{k}\right)^{\ell}\left((i k)^{m / 2}(\varphi(i k) \mathcal{A}(i k))\right) \mid k \in \mathbb{R}_{k_{0}}\right\}\right) \leq C\|\varphi\|_{H_{\infty}^{1}(\mathbb{R})} r_{b}, \\
\mathcal{R}_{\mathcal{L}\left(\mathcal{X}_{q}\left(B_{R}\right), L_{q}\left(B_{R}\right)^{N}\right)}\left(\left\{\left(k \partial_{k}\right)^{\ell} \nabla(\varphi(i k) \mathcal{P}(i k)) \mid k \in \mathbb{R}_{k_{0}}\right\}\right) \leq C\|\varphi\|_{H_{\infty}^{1}(\mathbb{R})} r_{b}, \\
\mathcal{R}_{\mathcal{L}\left(\mathcal{X}_{q}\left(B_{R}\right), W_{q}^{3-n-1 / q}\left(S_{R}\right)\right)}\left(\left\{\left(k \partial_{k}\right)^{\ell}\left((i k)^{n}(\varphi(i k) \mathcal{H}(i k))\right) \mid k \in \mathbb{R}_{k_{0}}\right\}\right) \leq C\|\varphi\|_{H_{\infty}^{1}(\mathbb{R})} r_{b}
\end{array}
$$

for $\ell=0,1, m=0,1,2$ and $n=0,1$. To prove (4.5), we use the following lemma concerning the fundamental properties of the $\mathcal{R}$-bound and scalar-valued Fourier multipliers.

Lemma 13. (a) Let $X$ and $Y$ be Banach spaces, and let $\mathcal{T}$ and $\mathcal{S}$ be $\mathcal{R}$-bounded families in $\mathcal{L}(X, Y)$. Then, $\mathcal{T}+\mathcal{S}=\{T+S \mid T \in \mathcal{T}, S \in \mathcal{S}\}$ is also an $\mathcal{R}$-bounded family in $\mathcal{L}(X, Y)$ and

$$
\mathcal{R}_{\mathcal{L}(X, Y)}(\mathcal{T}+\mathcal{S}) \leq \mathcal{R}_{\mathcal{L}(X, Y)}(\mathcal{T})+\mathcal{R}_{\mathcal{L}(X, Y)}(\mathcal{S})
$$

(b) Let $X, Y$ and $Z$ be Banach spaces, and let $\mathcal{T}$ and $\mathcal{S}$ be $\mathcal{R}$-bounded families in $\mathcal{L}(X, Y)$ and $\mathcal{L}(Y, Z)$, respectively. Then, $\mathcal{S} \mathcal{T}=\{S T \mid T \in \mathcal{T}, S \in \mathcal{S}\}$ is also an $\mathcal{R}$-bounded family in $\mathcal{L}(X, Z)$ and

$$
\mathcal{R}_{\mathcal{L}(X, Z)}(\mathcal{S T}) \leq \mathcal{R}_{\mathcal{L}(X, Y)}(\mathcal{T}) \mathcal{R}_{\mathcal{L}(Y, Z)}(\mathcal{S})
$$

(c) Let $1<p, q<\infty$ and let $D$ be a domain in $\mathbb{R}^{N}$. Let $m=m(\lambda)$ be a bounded function defined on a subset $U$ of $\mathbb{C}$ and let $M_{m}(\lambda)$ be a map defined by

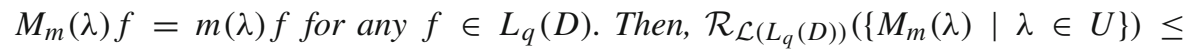
$C_{N, q, D}\|m\|_{L_{\infty}(U)}$.

(d) Let $n=n(\tau)$ be a $C^{1}$-function defined on $\mathbb{R} \backslash\{0\}$ that satisfies the conditions $|n(\tau)| \leq \gamma$ and $\left|\tau n^{\prime}(\tau)\right| \leq \gamma$ with some constant $c>0$ for any $\tau \in \mathbb{R} \backslash\{0\}$. Let $T_{n}$ be an operator-valued Fourier multiplier defined by $T_{n} f=\mathcal{F}^{-1}[n \mathcal{F}[f]]$ for any $f$ 
with $\mathcal{F}[f] \in \mathcal{D}\left(\mathbb{R}, L_{q}(D)\right)$. Then, $T_{n}$ is extended to a bounded linear operator from $L_{p}\left(\mathbb{R}, L_{q}(D)\right)$ into itself. Moreover, denoting this extension also by $T_{n}$, we have

$$
\left\|T_{n}\right\|_{\mathcal{L}\left(L_{p}\left(\mathbb{R}, L_{q}(D)\right)\right)} \leq C_{p, q, D} \gamma .
$$

Here, we only prove the $\mathcal{R}$-boundedness of $\varphi(i k) i k \mathcal{A}(i k)$. The $\mathcal{R}$-boundedness of the other terms can be proved by the same argument. Let $n \in \mathbb{N},\left\{k_{\ell}\right\}_{\ell=1}^{n} \in \mathbb{R}^{n}$, $\left\{F_{\ell}\right\}_{\ell=1}^{n} \in \mathcal{X}_{q}\left(B_{R}\right)^{n}$. Changing the labeling of indices if necessary, we may assume that $\varphi\left(k_{\ell}\right) \neq 0$ for $k=1, \ldots, m$ and $\varphi\left(k_{\ell}\right)=0$ for $\ell=m+1, \ldots, n$. And then, using Lemma 13, we have

$$
\begin{aligned}
& \left\|\sum_{\ell=1}^{n} r_{\ell} \varphi\left(i k_{\ell}\right)\left(i k_{\ell}\right) \mathcal{A}\left(i k_{\ell}\right) F_{\ell}\right\|_{L_{q}\left((0,1), L_{q}\left(B_{R}\right)\right)} \\
& \quad=\left\|\sum_{\ell=1}^{m} r_{\ell} \varphi\left(i k_{\ell}\right)\left(i k_{\ell}\right) \mathcal{A}\left(i k_{\ell}\right) F_{\ell}\right\|_{L_{q}\left((0,1), L_{q}\left(B_{R}\right)\right)} \\
& \quad \leq r_{b}\left\|\sum_{\ell=1}^{m} r_{\ell} \varphi\left(i k_{\ell}\right) F_{\ell}\right\|_{L_{q}\left((0,1), L_{q}\left(B_{R}\right)\right)} \\
& \quad=r_{b}\left\|\sum_{\ell=1}^{n} r_{\ell} \varphi\left(i k_{\ell}\right) F_{\ell}\right\|_{L_{q}\left((0,1), L_{q}\left(B_{R}\right)\right)} \\
& \quad \leq C_{q, R}\|\varphi\|_{H_{\infty}^{1}\left(B_{R}\right)} r_{b}\left\|\sum_{\ell=1}^{n} r_{\ell} F_{\ell}\right\|_{L_{q}\left((0,1), L_{q}\left(B_{R}\right)\right)},
\end{aligned}
$$

which shows that

$$
\mathcal{R}_{\mathcal{L}\left(\mathcal{X}_{q}\left(B_{R}\right), L_{q}\left(B_{R}\right)^{N}\right)}\left(\left\{i k \varphi(i k) \mathcal{A}(i k) \mid k \in \mathbb{R}_{k_{0}}\right\}\right) \leq C_{q, R}\|\varphi\|_{H_{\infty}^{1}(\mathbb{R})} r_{b} .
$$

For $f \in\{\mathbf{F}, G, \mathbf{G}, D, \mathbf{H}\}$, let

$$
f_{\psi}=\mathcal{F}_{\mathbb{T}}^{-1}\left[\psi \mathcal{F}_{\mathbb{T}}[f]\right]
$$

We consider the high frequency part of the equations (4.1):

$$
\begin{aligned}
\partial_{t} \mathbf{u}_{\psi}-\operatorname{Div}\left(\mu \mathbf{D}\left(\mathbf{u}_{\psi}\right)-\mathfrak{p}_{\psi} \mathbf{I}\right)=\mathbf{F}_{\psi} & \text { in } B_{R} \times(0,2 \pi), \\
\operatorname{div} \mathbf{u}_{\psi}=G_{\psi}=\operatorname{div} \mathbf{G}_{\psi} & \text { in } B_{R} \times(0,2 \pi), \\
\partial_{t} \rho_{\psi}+\mathcal{M} \rho_{\psi}-\left(\mathcal{A} \mathbf{u}_{\psi}\right) \cdot \mathbf{n}=D_{\psi} & \text { on } S_{R} \times(0,2 \pi), \\
\left(\mu \mathbf{D}\left(\mathbf{u}_{\psi}\right)-\mathfrak{p}_{\psi} \mathbf{I}\right) \mathbf{n}-\left(\mathcal{B}_{R} \rho_{\psi}\right) \mathbf{n}=\mathbf{H}_{\psi} & \text { on } S_{R} \times(0,2 \pi) .
\end{aligned}
$$

By Theorem 8, Theorem 9, and (4.5), we have immediately the following theorem.

Theorem 14. Let $1<p, q<\infty$. Then, for any functions $\mathbf{F}, G, \mathbf{G}, D$, and $\mathbf{H}$ with

$$
\begin{aligned}
& \mathbf{F} \in L_{p, \operatorname{per}}\left((0,2 \pi), L_{q}\left(B_{R}\right)^{N}\right), \quad D \in L_{p, \operatorname{per}}\left((0,2 \pi), W_{q}^{2-1 / q}\left(B_{R}\right)\right), \\
& \mathbf{H} \in H_{p, \operatorname{per}}^{1 / 2}\left((0,2 \pi), L_{q}\left(B_{R}\right)^{N}\right) \cap L_{p, \operatorname{per}}\left((0,2 \pi), H_{q}^{1}\left(B_{R}\right)^{N}\right),
\end{aligned}
$$




$$
\begin{aligned}
& G \in H_{p, \text { per }}^{1 / 2}\left((0,2 \pi), L_{q}\left(B_{R}\right)\right) \cap L_{p, \operatorname{per}}\left((0,2 \pi), H_{q}^{1}\left(B_{R}\right)\right), \\
& \quad \mathbf{G} \in H_{p, \text { per }}^{1}\left((0,2 \pi), L_{q}\left(B_{R}\right)^{N}\right),
\end{aligned}
$$

We let

$$
\begin{aligned}
\mathbf{u}_{\psi} & =\mathcal{F}_{\mathbb{T}}^{-1}\left[\varphi(i k) \mathcal{A}(i k) \mathcal{F}_{k}\left(\mathbf{F}_{\psi}, D_{\psi}, \mathbf{H}_{\psi}, G_{\psi}, \mathbf{G}_{\psi}\right)\right](\cdot, t), \\
\mathfrak{p}_{\psi} & =\mathcal{F}_{\mathbb{T}}^{-1}\left[\varphi(i k) \mathcal{P}(i k) \mathcal{F}_{k}\left(\mathbf{F}_{\psi}, D_{\psi}, \mathbf{H}_{\psi}, G_{\psi}, \mathbf{G}_{\psi}\right)\right](\cdot, t), \\
\rho_{\psi} & =\mathcal{F}_{\mathbb{T}}^{-1}\left[\varphi(i k) \mathcal{A}(i k) \mathcal{F}_{k}\left(\mathbf{F}_{\psi}, D_{\psi}, \mathbf{H}_{\psi}, G_{\psi}, \mathbf{G}_{\psi}\right)\right](\cdot, t),
\end{aligned}
$$

where we have set

$$
\begin{aligned}
\mathcal{F}_{k}\left(\mathbf{F}_{\psi}, D_{\pi}, \mathbf{H}_{\psi}, G_{\psi}, \mathbf{G}_{\psi}\right)= & \psi(i k)\left(\mathcal{F}_{\mathbb{T}}[\mathbf{F}](i k), \mathcal{F}_{\mathbb{T}}[D](i k),(i k)^{1 / 2} \mathcal{F}_{\mathbb{T}}[\mathbf{H}](i k), \mathcal{F}_{\mathbb{T}}[\mathbf{H}](i k),\right. \\
& \left.(i k)^{1 / 2} \mathcal{F}_{\mathbb{T}}[G](i k), \mathcal{F}_{\mathbb{T}}[G](i k), i k \mathcal{F}_{\mathbb{T}}[\mathbf{G}](i k)\right) .
\end{aligned}
$$

Then, $\mathbf{u}_{\psi}, \mathfrak{p}_{\psi}$ and $\rho_{\psi}$ are the unique solutions of equations (4.6), which possess the following estimate:

$$
\begin{aligned}
& \left\|\mathbf{u}_{\psi}\right\|_{L_{p}\left((0,2 \pi), H_{q}^{2}\left(B_{R}\right)\right)}+\left\|\partial_{t} \mathbf{u}_{\psi}\right\|_{L_{p}\left((0,2 \pi), L_{q}\left(B_{R}\right)\right)}+\left\|\nabla \mathfrak{p}_{\psi}\right\|_{L_{p}\left((0,2 \pi), L_{q}\left(B_{R}\right)\right)} \\
& \quad+\left\|\rho_{\psi}\right\|_{L_{p}\left((0,2 \pi), W_{q}^{3-1 / q}\left(S_{R}\right)\right)}+\left\|\partial_{t} \rho_{\psi}\right\|_{H_{p}^{1}\left((0,2 \pi), W_{q}^{2-1 / q}\left(S_{R}\right)\right)} \\
& \quad \leq C\left\{\left\|\mathbf{F}_{\psi}\right\|_{L_{p}\left((0,2 \pi), L_{q}\left(B_{R}\right)\right)}\right. \\
& \quad+\left\|D_{\psi}\right\|_{L_{p}\left((0,2 \pi), W_{q}^{2-1 / q}\left(S_{R}\right)\right)}+\left\|\Lambda^{1 / 2}\left(G_{\psi}, \mathbf{H}_{\psi}\right)\right\|_{L_{p}\left((0,2 \pi), L_{q}\left(B_{R}\right)\right)} \\
& \left.\quad+\left\|\left(G_{\psi}, \mathbf{H}_{\psi}\right)\right\|_{L_{p}\left((0,2 \pi), H_{q}^{1}\left(B_{R}\right)\right)}+\left\|\partial_{t} \mathbf{G}_{\psi}\right\|_{L_{p}\left((0,2 \pi), L_{q}\left(B_{R}\right)\right)}\right\}
\end{aligned}
$$

for some constant $C>0$. Here, we have set

$$
\Lambda^{1 / 2}\left(G_{\psi}, \mathbf{H}_{\psi}\right)=\mathcal{F}_{\mathbb{T}}^{-1}\left[(i k)^{1 / 2} \psi(i k)\left(\mathcal{F}_{\mathbb{T}}[G](i k), \mathcal{F}_{\mathbb{T}}[\mathbf{H}](i k)\right)\right] .
$$

We now consider the lower frequency part of solutions of equations (4.1). Namely, we consider equations (4.3) for $k \in \mathbb{R}$ with $1 \leq|k|<k_{0}+4$. We shall show the following theorem.

Theorem 15. Let $1<q<\infty$ and $k \in \mathbb{Z}$ with $1 \leq|k| \leq k_{0}+3$. Then, for any $\mathbf{f} \in L_{q}\left(B_{R}\right)^{N}, g \in H_{q}^{1}\left(B_{R}\right), d \in W_{q}^{2-1 / q}\left(S_{R}\right), \mathbf{h} \in H_{q}^{1}\left(B_{R}\right)^{N}$, and $\mathbf{g} \in L_{q}\left(B_{R}\right)^{N}$, problem (4.3) admits unique solutions $\mathbf{v} \in H_{q}^{2}\left(B_{R}\right)^{N}, \mathfrak{q} \in H_{q}^{1}\left(B_{R}\right)$, and $\eta \in W_{q}^{3-1 / q}\left(S_{R}\right)$ possessing the estimate:

$$
\begin{aligned}
& \|\mathbf{v}\|_{H_{q}^{2}\left(B_{R}\right)}+\|\nabla \mathfrak{q}\|_{L_{q}\left(B_{R}\right)}+\|\eta\|_{W_{q}^{3-1 / q}\left(S_{R}\right)} \\
& \quad \leq C\left(\|\mathbf{f}\|_{L_{q}\left(B_{R}\right)}+\|d\|_{W_{q}^{2-1 / q}\left(S_{R}\right)}+\|(g, \mathbf{h})\|_{H_{q}^{1}\left(B_{R}\right)}+\|\mathbf{g}\|_{L_{q}\left(B_{R}\right)}\right)
\end{aligned}
$$

for some constant $C>0$.

Proof. From Theorem 11, problem (4.3) with $k=k_{0}+4$ admits unique solutions $\mathbf{v}_{k_{0}} \in H_{q}^{2}\left(B_{R}\right)^{N}, \mathfrak{q}_{k_{0}} \in H_{q}^{1}\left(B_{R}\right)$, and $\eta_{k_{0}} \in W_{q}^{3-1 / q}\left(S_{R}\right)$ possessing the estimate:

$$
\begin{aligned}
& \left\|\mathbf{v}_{k_{0}}\right\|_{H_{q}^{2}\left(B_{R}\right)}+\left\|\nabla \mathfrak{q}_{k_{0}}\right\|_{L_{q}\left(B_{R}\right)}+\left\|\eta_{k_{0}}\right\|_{W_{q}^{3-1 / q}\left(S_{R}\right)} \\
& \quad \leq C\left(\|\mathbf{f}\|_{L_{q}\left(B_{R}\right)}+\|d\|_{W_{q}^{2-1 / q}\left(S_{R}\right)}+\|(g, \mathbf{h})\|_{H_{q}^{1}\left(B_{R}\right)}+\|\mathbf{g}\|_{L_{q}\left(B_{R}\right)}\right)
\end{aligned}
$$


for some constant $C$. Thus, for any $k \in \mathbb{R}$ with $|k|<k_{0}+4$, we consider the unique solvability of the equations:

$$
\begin{aligned}
i k \mathbf{w}-\operatorname{Div}(\mu \mathbf{D}(\mathbf{w})-\mathfrak{r} \mathbf{I})=\mathbf{f}, \quad \operatorname{div} \mathbf{w}=0 & \text { in } B_{R}, \\
i k \zeta+\mathcal{M} \zeta-(\mathcal{A w}) \cdot \mathbf{n}=d & \text { on } S_{R}, \\
(\mu \mathbf{D}(\mathbf{w})-\mathfrak{r} \mathbf{I}) \mathbf{n}-\sigma\left(\mathcal{B}_{R} \zeta\right) \mathbf{n}=0 & \text { on } S_{R},
\end{aligned}
$$

where we have set $\mathbf{f}=i\left(k-k_{0}\right) \mathbf{v}_{k_{0}}$ and $d=i\left(k_{0}-k\right) \eta_{k_{0}}$. In fact, if we set $\mathbf{v}=\mathbf{v}_{k_{0}}+\mathbf{w}$, $\mathfrak{q}=\mathfrak{q}_{k_{0}}+\mathfrak{r}$, and $\eta=\eta_{k_{0}}+\zeta$, then $\mathbf{v}, \mathfrak{q}$ and $\eta$ are unique solutions of equations (4.3).

In what follows, we study the unique solvability of equations (4.9) in the case where $\mathbf{f} \in L_{q}\left(B_{R}\right)$ and $d \in W_{q}^{2-1 / q}\left(S_{R}\right)$ are arbitrary. To solve (4.9), it is convenient to study the functional analytic form of (4.9), and so we eliminate the pressure term $\mathfrak{r}$ and the divergence condition $\operatorname{div} \mathbf{w}=0$ in $B_{R}$. Given $\mathbf{v} \in H_{q}^{2}\left(B_{R}\right)^{N}$ and $\zeta \in W_{q}^{3-1 / q}\left(S_{R}\right)$, let $K=K(\mathbf{v}, \zeta) \in H_{q}^{1}\left(B_{R}\right)$ be the unique solution of the weak Dirichlet problem:

$$
(\nabla K, \nabla \varphi)_{B_{R}}=(\operatorname{Div}(\mu \mathbf{D}(\mathbf{v}))-\nabla \operatorname{div} \mathbf{v}, \nabla \varphi)_{B_{R}} \quad \text { for any } \varphi \in \hat{H}_{q^{\prime}, 0}^{1}\left(B_{R}\right)
$$

subject to

$$
K=<\mu \mathbf{D}(\mathbf{v}) \mathbf{n}, \mathbf{n}>-\sigma \mathcal{B} \zeta-\operatorname{div} \mathbf{v} \quad \text { on } S_{R},
$$

where we have set

$$
\hat{H}_{q^{\prime}, 0}^{1}\left(B_{R}\right)=\left\{\varphi \in L_{q, \operatorname{loc}}\left(B_{R}\right)\left|\nabla \varphi \in L_{q}\left(B_{R}\right)^{N}, \quad \varphi\right|_{S_{R}}=0\right\}
$$

and $q^{\prime}=q /(q-1)$. In view of Poincaré's inequality, $\hat{H}_{q^{\prime}, 0}^{1}\left(B_{R}\right)=H_{q^{\prime}, 0}^{1}\left(B_{R}\right)=\{\varphi \in$ $\left.H_{q^{\prime}}^{1}\left(B_{R}\right)|\varphi|_{S_{R}}=0\right\}$. Instead of (4.9), we consider the equations:

$$
\begin{aligned}
i k \mathbf{w}-\operatorname{Div}(\mu \mathbf{D}(\mathbf{w})-K(\mathbf{w}, \zeta) \mathbf{I})=\mathbf{f} & \text { in } B_{R}, \\
i k \zeta+\mathcal{M} \zeta-(\mathcal{A} \mathbf{w}) \cdot \mathbf{n}=d & \text { on } S_{R}, \\
(\mu \mathbf{D}(\mathbf{w})-K(\mathbf{w}, \zeta) \mathbf{I}) \mathbf{n}-\sigma\left(\mathcal{B}_{R} \zeta\right) \mathbf{n}=0 & \text { on } S_{R}
\end{aligned}
$$

In view of the boundary condition (4.11) for $K(\mathbf{w}, \zeta)$, that $\mathbf{w}$ and $\zeta$ satisfy the third equation of equations (4.12) is equivalent to

$$
(\mu \mathbf{D}(\mathbf{w}) \mathbf{n})_{\tau}=0 \text { and } \operatorname{div} \mathbf{w}=0 \quad \text { on } S_{R}
$$

where $\mathbf{d}_{\tau}=\mathbf{d}-<\mathbf{d}, \mathbf{n}>\mathbf{n}$ for any $N$-vector $\mathbf{d}$. Let $J_{q}\left(B_{R}\right)$ be a solenoidal space defined by setting

$$
J_{q}\left(B_{R}\right)=\left\{\mathbf{v} \in L_{q}\left(B_{R}\right) \mid(\mathbf{v}, \nabla \varphi)_{B_{R}}=0 \text { for any } \varphi \in \hat{H}_{q^{\prime}, 0}^{1}\left(B_{R}\right)\right\} .
$$

Obviously, for $\mathbf{v} \in H_{q}^{1}\left(B_{R}\right)$, in order that $\operatorname{div} \mathbf{v}=0$ in $B_{R}$, it is necessary and sufficient that $\mathbf{v} \in J_{q}\left(B_{R}\right)$. For any $\mathbf{f} \in L_{q}\left(B_{R}\right)^{N}$, let $\psi \in H_{q, 0}^{1}\left(B_{R}\right)$ be a unique solution of the weak Dirichlet problem:

$$
(\nabla \psi, \nabla \varphi)_{B_{R}}=(\mathbf{f}, \nabla \varphi)_{B_{R}} \quad \text { for any } \varphi \in \hat{H}_{q^{\prime}, 0}^{1}\left(B_{R}\right)
$$


Let $\mathbf{g}=\mathbf{f}-\nabla \psi$ and inserting this formula into equations (4.9), we have

$$
\begin{aligned}
i k \mathbf{w}-\operatorname{Div}(\mu \mathbf{D}(\mathbf{w})-(\mathfrak{r}-\psi) \mathbf{I})=\mathbf{g}, \quad \operatorname{div} \mathbf{w}=0 & \text { in } B_{R}, \\
i k \zeta+\mathcal{M} \zeta-(\mathcal{A} \mathbf{w}) \cdot \mathbf{n}=d & \text { on } S_{R}, \\
(\mu \mathbf{D}(\mathbf{w})-(\mathfrak{r}-\psi) \mathbf{I}) \mathbf{n}-\sigma\left(\mathcal{B}_{R} \zeta\right) \mathbf{n}=0 & \text { on } S_{R} .
\end{aligned}
$$

where we have used the fact that $\left.\psi\right|_{S_{R}}=0$. Therefore, we shall solve equations (4.9) for $\mathbf{f} \in J_{q}\left(B_{R}\right)$ and $d \in W_{q}^{2-1 / q}\left(S_{R}\right)$. When $\mathbf{f} \in J_{q}\left(B_{R}\right)$, the equations (4.9) and (4.12) are equivalent. In fact, if $\mathbf{w} \in H_{q}^{2}\left(B_{R}\right)^{N}$ and $\zeta \in W_{q}^{3-1 / q}\left(S_{R}\right)$ satisfy equations (4.9) with some $\mathfrak{r} \in H_{q}^{1}\left(B_{R}\right)$. Then, for any $\varphi \in \hat{H}_{q^{\prime}, 0}^{1}\left(B_{R}\right)$, we have

$$
\begin{aligned}
0 & =(\mathbf{f}, \nabla \varphi)_{B_{R}}=(i k \mathbf{w}-\operatorname{Div}(\mu \mathbf{D}(\mathbf{w})), \nabla \varphi)_{B_{R}}+(\nabla \mathfrak{r}, \nabla \varphi)_{B_{R}} \\
& =(\nabla(\mathfrak{r}-K(\mathbf{w}, \zeta)), \nabla \varphi)_{B_{R}},
\end{aligned}
$$

where we have used the fact that $\operatorname{div} \mathbf{w}=0$. Moreover, from the boundary conditions in equations (4.9) and (4.11), it follows that

$$
\mathfrak{r}-K(\mathbf{w}, \zeta)=<\mu \mathbf{D}(\mathbf{w}) \mathbf{n}, \mathbf{n}>-\sigma \mathcal{B}_{R} \zeta-K(\mathbf{w}, \zeta)=\operatorname{div} \mathbf{w}=0
$$

on $S_{R}$ because $\operatorname{div} \mathbf{w}=0$. Thus, the uniqueness of the solutions to his weak Dirichlet problem yields that $\mathfrak{r}=K(\mathbf{w}, \zeta)$, and so $\mathbf{w}$ and $\zeta$ satisfy equations (4.12). Conversely, let $\mathbf{w} \in H_{q}^{2}\left(B_{R}\right)^{N}$ and $\zeta \in W_{q}^{3-1 / q}\left(S_{R}\right)$ be solutions of equations (4.12). For any $\varphi \in \hat{H}_{q^{\prime}, 0}^{1}\left(B_{R}\right)$, we have

$$
\begin{aligned}
0 & =(\mathbf{f}, \nabla \varphi)_{B_{R}}=i k(\mathbf{w}, \nabla \varphi)_{B_{R}}-(\operatorname{Div}(\mu \mathbf{D}(\mathbf{w})), \nabla \varphi)_{B_{R}}+(\nabla K(\mathbf{w}, \zeta), \nabla \varphi)_{B_{R}} \\
& =-i k(\operatorname{div} \mathbf{w}, \varphi)_{B_{R}}-(\nabla \operatorname{div} \mathbf{w}, \nabla \varphi)_{B_{R}}
\end{aligned}
$$

Moreover, from the boundary condition (4.13) it follows that div $\mathbf{w}=0$ on $S_{R}$. The uniqueness implies that $\operatorname{div} \mathbf{w}=0$ in $B_{R}$. Thus, $\mathbf{w}, \mathfrak{r}=K(\mathbf{w}, \zeta)$ and $\zeta$ are solutions of equations (4.9). In particular, for solutions $\mathbf{w}$ and $\zeta$ of equations (4.12), we see that $\mathbf{w}$ satisfies the divergence condition: $\operatorname{div} \mathbf{w}=0$ in $B_{R}$ automatically.

From now on, we study the unique existence theorem for equations (4.12) for any $\mathbf{f} \in J_{q}\left(B_{R}\right)$ and $d \in W_{q}^{2-1 / q}\left(S_{R}\right)$. To formulate problem (4.12) in a functional analytic setting, we define the spaces $\mathcal{H}_{q}, \mathcal{D}_{q}$ and the operator $\mathbf{A}$ by setting

$$
\begin{aligned}
\mathcal{H}_{q} & =\left\{(\mathbf{f}, d) \mid \mathbf{f} \in J_{q}\left(B_{R}\right), \quad d \in W_{q}^{2-1 / q}\left(S_{R}\right)\right\}, \\
\mathcal{D}_{q} & =\left\{(\mathbf{w}, \zeta) \in \mathcal{H}_{q}\left|\mathbf{w} \in H_{q}^{2}\left(B_{R}\right)^{N}, \quad \zeta \in W_{q}^{3-1 / q}\left(S_{R}\right), \quad(\mu \mathbf{D}(\mathbf{w}))_{\tau}\right|_{S_{R}}=0\right\}, \\
\mathbf{A} U & =\left(\operatorname{Div}(\mu \mathbf{D}(\mathbf{w})-K(\mathbf{w}, \zeta) \mathbf{I}),\left.(-\mathcal{M} \zeta+(\mathcal{A} \mathbf{w}) \cdot \mathbf{n})\right|_{S_{R}}\right) \quad \text { for } U=(\mathbf{w}, \zeta) \in \mathcal{D}_{q},
\end{aligned}
$$

where we have used (4.13) and $\operatorname{div} \mathbf{w}=0$ in the definition of $\mathcal{D}_{q}$. We write equations (4.12) as

$$
i k U-\mathbf{A} U=F \quad \text { in } \mathcal{H}_{q} .
$$

In view of Theorem 11, we see that $k=k_{0}+4$ is an element of the resolvent set of the operator $\mathbf{A}$, and so $\left(i\left(k_{0}+4\right) \mathbf{I}-\mathbf{A}\right)^{-1}$ exists in $\mathcal{L}\left(\mathcal{H}_{q}, \mathcal{D}_{q}\right)$. Since $B_{R}$ is a 
compact set, it follows from the Rellich compactness theorem that $\left(i\left(k_{0}+4\right) \mathbf{I}-\mathbf{A}\right)^{-1}$ is a compact operator from $\mathcal{H}_{q}$ into itself. Thus, in view of Riesz-Schauder theory, in particular, Fredholm alternative principle, that $k$ belongs to the resolvent set if and only if uniqueness holds for $k$. Thus, our task is to prove the uniqueness of solutions to equations (4.14). Let $U=(\mathbf{w}, \zeta) \in \mathcal{D}_{q}$ satisfy the homogeneous equations:

$$
i k U-\mathbf{A} U=0 \text { in } \mathcal{H}_{q} \text {. }
$$

Namely, $(\mathbf{w}, \zeta) \in \mathcal{D}_{q}$ satisfies equations:

$$
\begin{array}{rc}
i k \mathbf{w}-\operatorname{Div}(\mu \mathbf{D}(\mathbf{w})-K(\mathbf{w}, \zeta) \mathbf{I})=0 & \text { in } B_{R}, \\
i k \zeta+\mathcal{M} \zeta-(\mathcal{A} \mathbf{w}) \cdot \mathbf{n}=0 & \text { on } S_{R} \\
(\mu \mathbf{D}(\mathbf{w})-K(\mathbf{w}, \zeta) \mathbf{I}) \mathbf{n}-\sigma\left(\mathcal{B}_{R} \zeta\right) \mathbf{n}=0 & \text { on } S_{R}
\end{array}
$$

We first prove that

$$
(\zeta, 1)_{S_{R}}=0, \quad\left(\zeta, x_{j}\right)_{S_{R}}=0 \text { for } j=1, \ldots, N
$$

Integrating the second equation of equations (4.16) and applying the divergence theorem of Gauss gives that

$$
0=i k(\zeta, 1)_{S_{R}}+(\zeta, 1)_{S_{R}}\left|S_{R}\right|-\int_{B_{R}} \operatorname{div} \mathcal{A} \mathbf{w} \mathrm{d} x=\left(i k+\left|S_{R}\right|\right)(\zeta, 1)_{S_{R}},
$$

where we have set $\left|S_{R}\right|=\int_{S_{R}} \mathrm{~d} \omega$ and we have used the fact that $\operatorname{div} \mathbf{w}=0$ in $B_{R}$. Thus, we have $(\zeta, 1)_{S_{R}}=0$. Multiplying the second equation of equations (4.16) with $x_{j}$, integrating the resultant formula over $S_{R}$ and using the divergence theorem of Gauss gives that

$$
0=i k\left(\zeta, x_{\ell}\right)_{S_{R}}+\left(\zeta, x_{\ell}\right)_{S_{R}}\left(x_{\ell}, x_{\ell}\right)_{S_{R}}-\int_{B_{R}} \operatorname{div}\left(x_{\ell} \mathcal{A} \mathbf{w}\right) \mathrm{d} x,
$$

because $\left(x_{j}, x_{\ell}\right)_{S_{R}}=0$ for $j \neq \ell$. Since

$$
\int_{B_{R}} \operatorname{div}\left(x_{\ell} \mathcal{A} \mathbf{w}\right) \mathrm{d} x=\int_{B_{R}}\left(\mathbf{w}_{\ell}-\frac{1}{\left|B_{R}\right|} \int_{B_{R}} \mathbf{w}_{\ell} \mathrm{d} x\right) \mathrm{d} x=0,
$$

we have $\left(\zeta, x_{\ell}\right)_{S_{R}}=0$, because $\left(x_{\ell}, x_{\ell}\right)_{S_{R}}=\left(R^{2} / N\right)\left|S_{R}\right|>0$. Thus, we have proved (4.17). In particular, $\mathcal{M} \zeta=0$ in (4.16).

We now prove that $\mathbf{w}=0$. For this purpose, we first consider the case where $2 \leq q<\infty$. Since $B_{R}$ is bounded, $\mathcal{D}_{q} \subset \mathcal{D}_{2}$. Multiplying the first equation of (4.16) with $\mathbf{w}$ and integrating the resultant formula over $B_{R}$ and using the divergence theorem of Gauss gives that

$$
0=i k\|\mathbf{w}\|_{L_{2}\left(B_{R}\right)}^{2}-\sigma\left(\mathcal{B}_{R} \zeta, \mathbf{n} \cdot \mathbf{w}\right)_{S_{R}}+\frac{\mu}{2}\|\mathbf{D}(\mathbf{w})\|_{L_{2}\left(B_{R}\right)}^{2},
$$


because $\operatorname{div} \mathbf{w}=0$ in $B_{R}$. By the second equation of (4.16) with $\mathcal{M} \zeta=0$, we have

$$
\sigma\left(\mathcal{B}_{R} \zeta, \mathbf{n} \cdot \mathbf{w}\right)_{S_{R}}=\sigma\left(\mathcal{B}_{R} \zeta, i k \zeta\right)_{S_{R}}+\sum_{k=1}^{N} \frac{1}{\left|B_{R}\right|} \int_{B_{R}} w_{j} \mathrm{~d} t\left(\mathcal{B}_{R} \zeta, R^{-1} x_{j}\right)_{S_{R}}
$$

where we have used $\mathbf{n}=R^{-1} x=R^{-1}\left(x_{1}, \ldots, x_{N}\right)$ for $x \in S_{R}$. Thus,

$$
\left(\mathcal{B}_{R} \zeta, x_{j}\right)_{S_{R}}=\left(\zeta,\left(\Delta_{S_{R}}+\frac{N-1}{R^{2}}\right) x_{j}\right)_{S_{R}}=0
$$

Moreover, since $\zeta$ satisfies (4.17), we know that

$$
-\left(\mathcal{B}_{R} \zeta, \zeta\right)_{S_{R}} \geq c\|\zeta\|_{L_{2}\left(S_{R}\right)}^{2}
$$

for some positive constant $c$, and therefore (4.18) implies $\mathbf{w}=0$.

Now the first equation of (4.16) yields $\nabla K(\mathbf{w}, \zeta)=0$, so that $K(\mathbf{w}, \zeta)$ is constant. Integration of the third equation of (4.16) over $S_{R}$ combined with (4.17) shows that this constant is 0 , that is, $K(\mathbf{w}, \zeta)=0$.

Finally, the third equation of (4.16) yields that $\mathcal{B}_{R} \zeta=0$ on $S_{R}$, and so by (4.17) we have $\zeta=0$. This completes the proof of the uniqueness in the case where $2 \leq q<\infty$. In particular, we have the unique existence theorem of solutions to equation (4.14).

We now consider the case where $1<q<2$. Let $\mathbf{f}$ be any element in $J_{q^{\prime}}\left(B_{R}\right)$ and let $V=(\mathbf{v}, \eta) \in \mathcal{D}_{q^{\prime}}$ be a solution of the equation:

$$
-i k V-\mathbf{A} V=(\mathbf{f}, 0) \text { in } \mathcal{H}_{q^{\prime}}
$$

The existence of such $V$ has already been proved above. Since $d=0$, we see that $\eta$ satisfies the relations:

$$
(\eta, 1)_{S_{R}}=0, \quad\left(\eta, x_{j}\right)_{S_{R}}=0 \text { for } j=1, \ldots, N
$$

and so $\mathcal{M} \eta=0$. Using the divergence theorem of Gauss, we have

$$
\begin{aligned}
(\mathbf{w}, \mathbf{f})_{B_{R}}= & (\mathbf{w},-i k \mathbf{v}-\operatorname{Div}(\mu \mathbf{D}(\mathbf{v})-K(\mathbf{v}, \eta) \mathbf{I}))_{B_{R}} \\
= & (i k \mathbf{w}, \mathbf{v})_{B_{R}}-(\mathbf{w},(\mu \mathbf{D}(\mathbf{v})-K(\mathbf{v}, \eta) \mathbf{I}) \mathbf{n})_{S_{R}}+\frac{\mu}{2}(\mathbf{D}(\mathbf{w}), \mathbf{D}(\mathbf{v}))_{B_{R}} \\
= & \left(\operatorname{Div}(\mu(\mathbf{D}(\mathbf{w})-K(\mathbf{w}, \zeta) \mathbf{I}), \mathbf{v})_{B_{R}}-\sigma\left(\mathbf{w} \cdot \mathbf{n}, \mathcal{B}_{R} \eta\right)_{S_{R}}+\frac{\mu}{2}(\mathbf{D}(\mathbf{w}), \mathbf{D}(\mathbf{v}))_{B_{R}}\right. \\
= & \sigma\left(\mathcal{B}_{R} \zeta, \mathbf{n} \cdot \mathbf{v}\right)_{S_{R}}-\sigma\left(\mathbf{w} \cdot \mathbf{n}, \mathcal{B}_{R} \eta\right)_{S_{R}} \\
= & \sigma\left(\mathcal{B}_{R} \zeta,-i k \eta+\frac{1}{\left|B_{R}\right|} \int_{B_{R}} \mathbf{v} \mathrm{d} y \cdot \mathbf{n}\right)_{S_{R}}-\sigma(i k \zeta \\
& \left.+\frac{1}{\left|B_{R}\right|} \int_{B_{R}} \mathbf{w} \mathrm{d} y \cdot \mathbf{n}, \mathcal{B}_{R} \eta\right)_{S_{R}} .
\end{aligned}
$$

Using the fact that $\left(\mathcal{B}_{R} \zeta, x_{j}\right)_{S_{R}}=\left(x_{j}, \mathcal{B}_{R} \eta\right)_{S_{R}}=0$, we have

$$
(\mathbf{w}, \mathbf{f})_{B_{R}}=\sigma i k\left(\mathcal{B}_{R} \zeta, \eta\right)_{S_{R}}-\sigma i k\left(\zeta, \mathcal{B}_{R} \eta\right)_{S_{R}}
$$




$$
\begin{aligned}
= & \sigma i k\left\{\frac{N-1}{R^{2}}(\zeta, \eta)_{S_{R}}-\left(\nabla_{S_{R}} \zeta, \nabla_{S_{R}} \eta\right)_{S_{R}}\right. \\
& \left.-\frac{N-1}{R^{2}}(\zeta, \eta)_{S_{R}}+\left(\nabla_{S_{R}} \zeta, \nabla_{S_{R}} \eta\right)_{S_{R}}\right\}=0 .
\end{aligned}
$$

For any $\mathbf{g} \in L_{q^{\prime}}\left(B_{R}\right)^{N}$, let $\psi \in \hat{H}_{q^{\prime}, 0}^{1}\left(B_{R}\right)$ be a unique solution of the weak Dirichlet problem:

$$
(\nabla \psi, \nabla \varphi)_{B_{R}}=(\mathbf{g}, \nabla \varphi)_{B_{R}} \text { for any } \varphi \in \hat{H}_{q, 0}^{1}\left(B_{R}\right) .
$$

Let $\mathbf{f}=\mathbf{g}-\nabla \psi$, and then $\mathbf{f} \in J_{q^{\prime}}\left(B_{R}\right)$, and so using the fact that $\mathbf{w} \in J_{q}\left(B_{R}\right)$, we have $(\mathbf{w}, \mathbf{g})_{B_{R}}=(\mathbf{w}, \mathbf{f})_{B_{R}}+(\mathbf{w}, \nabla \psi)_{B_{R}}=0$. The arbitrariness of $\mathbf{g} \in L_{q^{\prime}}\left(B_{R}\right)^{N}$ implies that $\mathbf{w}=0$. Thus, the second equation of (4.16) and (4.17) leads to $\zeta=0$. This completes the proof of the uniqueness in the case where $1<q<2$, and therefore the proof of Theorem 15 .

We now consider the linearized stationary problem:

$$
\begin{aligned}
\mathcal{L} \mathbf{v}-\operatorname{Div}(\mu \mathbf{D}(\mathbf{v})-\mathfrak{p} \mathbf{I})=\mathbf{f} & \text { in } B_{R}, \\
\operatorname{div} \mathbf{v}=g=\operatorname{div} \mathbf{g} & \text { in } B_{R}, \\
\mathcal{M} \rho-(\mathcal{A} \mathbf{v}) \cdot \mathbf{n}=d & \text { on } S_{R}, \\
(\mu \mathbf{D}(\mathbf{v})-\mathfrak{p} \mathbf{I}) \mathbf{n}-\sigma\left(\mathcal{B}_{R} \rho\right) \mathbf{n}=\mathbf{h} & \text { on } S_{R} .
\end{aligned}
$$

We shall prove the following theorem.

Theorem 16. Let $1<q<\infty$. Then, for any $\mathbf{f} \in L_{q}\left(B_{R}\right)^{N}, d \in W_{q}^{2-1 / q}\left(S_{R}\right)$, $g \in H_{q}^{1}\left(B_{R}\right), \mathbf{g} \in L_{q}\left(B_{R}\right)^{N}$, and $\mathbf{h} \in H_{q}^{1}\left(B_{R}\right)^{N}$, problem (4.19) admits unique solutions $\mathbf{v} \in H_{q}^{2}\left(B_{R}\right)^{N}, \mathfrak{p} \in H_{q}^{1}\left(B_{R}\right)$, and $\rho \in W_{q}^{3-1 / q}\left(S_{R}\right)$ possessing the estimate:

$$
\begin{aligned}
& \|\mathbf{v}\|_{H_{q}^{2}\left(B_{R}\right)}+\|\mathfrak{p}\|_{H_{q}^{1}\left(B_{R}\right)}+\|\rho\|_{W_{q}^{3-1 / q}\left(S_{R}\right)} \\
& \quad \leq C\left(\|\mathbf{f}\|_{L_{q}\left(B_{R}\right)}+\|d\|_{W_{q}^{2-1 / q}\left(S_{R}\right)}+\|(g, \mathbf{h})\|_{H_{q}^{1}\left(B_{R}\right)}+\|\mathbf{g}\|_{L_{q}\left(B_{R}\right)}\right)
\end{aligned}
$$

for some constant $C>0$.

Proof. The strategy of the proof is the same as that of Theorem 15. Since $\mathcal{L} \mathbf{v}, \mathcal{M} \rho$, and $\left|B_{R}\right|^{-1} \int_{B_{R}} \mathbf{v} \mathrm{d} y$ are lower order perturbations, choosing $k_{0}>0$ large enough, the generalized resolvent problem:

$$
\begin{aligned}
i k_{0} \mathbf{v}+\mathcal{L} \mathbf{v}-\operatorname{Div}(\mu \mathbf{D}(\mathbf{v})-\mathfrak{p} \mathbf{I})=\mathbf{f} & \text { in } B_{R}, \\
\operatorname{div} \mathbf{v}=g=\operatorname{div} \mathbf{g} & \text { in } B_{R}, \\
i k_{0} \rho+\mathcal{M} \rho-(\mathcal{A} \mathbf{v}) \cdot \mathbf{n}=d & \text { on } S_{R}, \\
(\mu \mathbf{D}(\mathbf{v})-\mathfrak{p} \mathbf{I}) \mathbf{n}-\sigma\left(\mathcal{B}_{R} \rho\right) \mathbf{n}=\mathbf{h} & \text { on } S_{R} .
\end{aligned}
$$

admits unique solutions: $\mathbf{v} \in H_{q}^{2}\left(B_{R}\right)^{N}, \mathfrak{p} \in H_{q}^{1}\left(B_{R}\right)$, and $\rho \in W_{q}^{3-1 / q}\left(S_{R}\right)$ possessing the estimate (4.20). Of course, the constant $C$ in (4.20) depends on $k_{0}$ in this case, 
but $k_{0}$ is fixed, and so we can say that $C$ in (4.20) is some fixed constant. The essential part of the proof is to show the unique existence of solutions to equations (4.19) with $g=\mathbf{g}=\mathbf{h}=0$, that is

$$
\begin{aligned}
\mathcal{L} \mathbf{v}-\operatorname{Div}(\mu \mathbf{D}(\mathbf{v})-\mathfrak{p} \mathbf{I})=\mathbf{f} & \text { in } B_{R}, \\
\operatorname{div} \mathbf{v}=0 & \text { in } B_{R}, \\
\mathcal{M} \rho-(\mathcal{A} \mathbf{v}) \cdot \mathbf{n}=d & \text { on } S_{R}, \\
(\mu \mathbf{D}(\mathbf{v})-\mathfrak{p} \mathbf{I}) \mathbf{n}-\sigma\left(\mathcal{B}_{R} \rho\right) \mathbf{n}=0 & \text { on } S_{R} .
\end{aligned}
$$

And then, the uniqueness of the reduced problem in the $L_{2}$ framework implies the unique existence of solutions as was studied in the proof Theorem 15. Thus, we define the reduced problem corresponding to equations (4.19). For $\mathbf{v} \in H_{q}^{2}\left(B_{R}\right)^{N}$ and $\rho \in W_{q}^{3-1 / q}\left(S_{R}\right)$, let $K=K(\mathbf{v}, \rho) \in H_{q}^{1}\left(B_{R}\right)$ be the unique solution of the weak Dirichlet problem:

$$
(\nabla K, \nabla \varphi)_{B_{R}}=\left(\operatorname{Div}(\mu \mathbf{D}(\mathbf{v})-\mathcal{L} \mathbf{v}-\nabla \operatorname{div} \mathbf{v}, \nabla \varphi)_{B_{R}} \quad \text { for any } \varphi \in \hat{H}_{q^{\prime}, 0}^{1}\left(B_{R}\right),\right.
$$

subject to the boundary condition:

$$
K=<\mu \mathbf{D}(\mathbf{v}) \mathbf{n}, \mathbf{n}>-\sigma \mathcal{B}_{R} \rho-\operatorname{div} \mathbf{v} \text { on } B_{R} .
$$

Then, the reduced problem corresponding to problem (4.19) with $g=\mathbf{g}=\mathbf{h}=0$ is given by the following equations:

$$
\begin{aligned}
\mathcal{L} \mathbf{v}-\operatorname{Div}(\mu \mathbf{D}(\mathbf{v})-K(\mathbf{v}, \rho) \mathbf{I})=\mathbf{f} & \text { in } B_{R}, \\
\mathcal{M} \rho-(\mathcal{A} \mathbf{v}) \cdot \mathbf{n}=d & \text { on } S_{R}, \\
(\mu \mathbf{D}(\mathbf{v})-K(\mathbf{v}, \rho) \mathbf{I}) \mathbf{n}-\sigma\left(\mathcal{B}_{R} \rho\right) \mathbf{n}=0 & \text { on } S_{R} .
\end{aligned}
$$

Then, for $\mathbf{f} \in J_{q}\left(B_{R}\right)$ and $d \in W_{q}^{2-1 / q}\left(S_{R}\right)$, problems (4.22) and (4.25) are equivalent. In fact, if problem (4.22) admits unique solutions $\mathbf{v} \in H_{q}^{2}\left(B_{R}\right)^{N}, \mathfrak{p} \in H_{q}^{1}\left(B_{R}\right)$ and $\rho \in W_{q}^{3-1 / q}\left(S_{R}\right)$, then for any $\varphi \in \hat{H}_{q^{\prime}, 0}^{1}\left(B_{R}\right)$, we have

$$
\begin{aligned}
0 & =(\mathbf{f}, \nabla \varphi)_{B_{R}}=(\mathcal{L} \mathbf{v}-\operatorname{Div}(\mu \mathbf{D}(\mathbf{v})), \nabla \varphi)_{B_{R}}+(\nabla \mathfrak{p}, \nabla \varphi)_{B_{R}} \\
& =(\nabla(\mathfrak{p}-K(\mathbf{v}, \rho)), \nabla \varphi)_{B_{R}}
\end{aligned}
$$

because div $\mathbf{v}=0$ in $B_{R}$. Moreover, from the boundary conditions in (4.22) and (4.24) it follows that

$$
\mathfrak{p}-K(\mathbf{v}, \rho)=<\mu \mathbf{D}(\mathbf{v}) \mathbf{n}, \mathbf{n}>-\sigma \mathcal{B}_{R} \rho-K(\mathbf{v}, \rho)=\operatorname{div} \mathbf{v}=0
$$

on $S_{R}$. The uniqueness of the weak Dirichlet problem leads to $\mathfrak{p}=K(\mathbf{v}, \rho)$, and therefore $\mathbf{v}$ and $\rho$ satisfy equations (4.25). Conversely, if $\mathbf{v} \in H_{q}^{2}\left(B_{R}\right)^{N}$ and $\rho \in$ $W_{q}^{3-1 / q}\left(S_{R}\right)$ satisfy the equations (4.25), then for any $\varphi \in \hat{H}_{q^{\prime}, 0}^{1}\left(B_{R}\right)$ we have

$$
0=(\mathbf{f}, \nabla \varphi)_{B_{R}}=\left(\mathcal{L} \mathbf{v}-\operatorname{Div}(\mu \mathbf{D}(\mathbf{v}), \nabla \varphi)_{B_{R}}+(\nabla K(\mathbf{v}, \rho), \nabla \varphi)_{B_{R}}=(\nabla \operatorname{div} \mathbf{v}, \nabla \varphi)_{B_{R}} .\right.
$$


Moreover, the boundary conditions of (4.25) and (4.24) gives that

$$
\operatorname{div} \mathbf{v}=<\mu \mathbf{D}(\mathbf{v}) \mathbf{n}, \mathbf{n}>-\sigma \mathcal{B}_{R} \rho-K(\mathbf{v}, \rho)=0 .
$$

The uniqueness of the weak Dirichlet problem yields that $\operatorname{div} \mathbf{v}=0$, and therefore, $\mathbf{v}$, $\mathfrak{p}=K(\mathbf{v}, \rho)$ and $\rho$ are solutions of equations (4.22).

Finally, we show the uniqueness of equations (4.21) in the $L_{2}$-framework, which yields Theorem 16 . Let $\mathbf{v} \in H_{2}^{2}\left(B_{R}\right)^{N}$ and $\rho \in W_{2}^{5 / 2}\left(S_{R}\right)$ satisfy the homogeneous equations:

$$
\begin{aligned}
\mathcal{L} \mathbf{v}-\operatorname{Div}(\mu \mathbf{D}(\mathbf{v})-K(\mathbf{v}, \rho) \mathbf{I})=0 & \text { in } B_{R}, \\
\mathcal{M} \rho-(\mathcal{A} \mathbf{v}) \cdot \mathbf{n}=0 & \text { on } S_{R}, \\
(\mu \mathbf{D}(\mathbf{v})-K(\mathbf{v}, \rho) \mathbf{I}) \mathbf{n}-\sigma\left(\mathcal{B}_{R} \rho\right) \mathbf{n}=0 & \text { on } S_{R} .
\end{aligned}
$$

Note that $\operatorname{div} \mathbf{v}=0$ in $B_{R}$. Employing the same argument as in the proof of Theorem 15 , we have

$$
(\rho, 1)_{S_{R}}=0, \quad\left(\rho, x_{j}\right)_{S_{R}}=0 \text { for } j=1, \ldots, N .
$$

In particular, $\mathcal{M} \rho=0$. Multiplying the first equation with $\mathbf{v}$, integrating the resultant formula on $B_{R}$ and using the divergence theorem of Gauss gives that

$$
0=(\mathcal{L} \mathbf{v}, \mathbf{v})_{B_{R}}+\sigma\left(\mathcal{B}_{R} \rho, \mathbf{n} \cdot \mathbf{v}\right)_{S_{R}}+\frac{\mu}{2}\|\mathbf{D}(\mathbf{v})\|_{L_{2}\left(B_{R}\right)}^{2},
$$

because $(K(\mathbf{v}, \rho), \operatorname{div} \mathbf{v})=0$ as follows from $\operatorname{div} \mathbf{v}=0$ in $B_{R}$. From (2.17) it follows that

$$
(\mathcal{L} \mathbf{v}, \mathbf{v})_{B_{R}}=\sum_{k=1}^{M}\left|\left(\mathbf{v}, \mathbf{p}_{k}\right)_{B_{R}}\right|^{2} .
$$

From the second equation of (4.26) with $\mathcal{M} \rho=0$ it follows that

$$
\left(\mathcal{B}_{R} \rho, \mathbf{n} \cdot \mathbf{v}\right)_{S_{R}}=\sum_{j=1}^{N} R^{-1}\left(\mathcal{B}_{R} \rho, x_{j}\right)_{S_{R}} \frac{1}{\left|B_{R}\right|} \int_{B_{R}} v_{j} \mathrm{~d} y=0 .
$$

Combining these formulas yields that

$$
0=\sum_{k=1}^{M}\left|\left(\mathbf{v}, \mathbf{p}_{k}\right)_{B_{R}}\right|^{2}+\frac{\mu}{2}\|\mathbf{D}(\mathbf{v})\|_{L_{2}\left(B_{R}\right)}^{2},
$$

which leads to $\mathbf{D}(\mathbf{v})=0$ and $\left(\mathbf{v}, \mathbf{p}_{k}\right)_{B_{R}}=0$ for $k=1, \ldots, M$. Thus, we have $\mathbf{v}=0$. From the first equation of (4.26), we have $\nabla K(\mathbf{v}, \rho)=0$, and so $K(\mathbf{v}, \rho)=c$ with some constant $c$. From the boundary condition of (4.26), we have $\sigma \mathcal{B} \rho=-c$ on $B_{R}$. Integrating this formula on $S_{R}$ and using the fact $(\rho, 1)_{S_{R}}=0$ in (4.27) gives that $c=0$. Thus, $\mathcal{B}_{R} \rho=0$ on $S_{R}$, but we know (4.27), and so

$$
0=-\left(\mathcal{B}_{R} \rho, \rho\right)_{S_{R}} \geq c\|\rho\|_{L_{2}\left(S_{R}\right)}^{2}
$$

for some constant $c>0$, which shows that $\rho=0$. This completes the proof of the uniqueness in the $L_{2}$ framework, the proof of Theorem 16. 
Proof of Theorem 6. We now prove Theorem 6. Let $\mathbf{u}_{\psi}, \mathfrak{p}_{\psi}$ and $\rho_{\psi}$ be functions given in Theorem 14 which are solutions of equations (4.6). Notice that $\psi(i k)=1$ for $|k| \geq k_{0}+4$ and $\psi(i k)=0$ for $|k| \leq k_{0}+3$. For $k \in \mathbb{Z}$ with $1 \leq|k| \leq k_{0}+3$, let

$$
\begin{gathered}
\mathbf{f}=\mathcal{F}_{\mathbb{T}}[\mathbf{F}](i k), \quad g=\mathcal{F}_{\mathbb{T}}[G](i k), \quad \mathbf{g}=\mathcal{F}_{\mathbb{T}}[\mathbf{G}](i k), \\
\quad d=\mathcal{F}_{\mathbb{T}}[D](i k), \quad \mathbf{h}=\mathcal{F}_{\mathbb{T}}[\mathbf{H}](i k)
\end{gathered}
$$

in equations (4.3), and we write solutions $\mathbf{v}, \mathfrak{q}$ and $\eta$ as $\mathbf{v}_{k}=\mathbf{v}, \mathfrak{q}_{k}=\mathfrak{q}$ and $\eta_{k}=\eta$. Let

$$
\mathbf{u}_{k}=\mathrm{e}^{i k t} \mathbf{v}_{k}, \quad \mathfrak{p}_{k}=\mathrm{e}^{i k t} \mathfrak{q}_{k}, \quad \rho_{k}=\mathrm{e}^{i k t} \eta_{k},
$$

and then, $\mathbf{u}_{k}, \mathfrak{p}_{k}$ and $\rho_{k}$ satisfy the equations:

$$
\begin{array}{rr}
\partial_{t} \mathbf{u}_{k}-\operatorname{Div}\left(\mu \mathbf{D}\left(\mathbf{u}_{k}\right)-\mathfrak{p}_{k} \mathbf{I}\right)=\mathrm{e}^{i k t} \mathcal{F}_{\mathbb{T}}[\mathbf{F}](i k) & \text { in } B_{R}, \\
\operatorname{div} \mathbf{u}_{k}=\mathrm{e}^{i k t} \mathcal{F}_{\mathbb{T}}[G](i k)=\operatorname{div}\left(\mathrm{e}^{i k t} \mathcal{F}_{\mathbb{T}}[\mathbf{G}](i k)\right) & \text { in } B_{R}, \\
\partial_{t} \rho_{k}+\mathcal{M} \rho_{k}-\left(\mathcal{A} \mathbf{u}_{k}\right) \cdot \mathbf{n}=\mathrm{e}^{i k t} \mathcal{F}_{\mathbb{T}}[D](i k) & \text { on } S_{R}, \\
\left(\mu \mathbf{D}\left(\mathbf{u}_{k}\right)-\mathfrak{p}_{k} \mathbf{I}\right) \mathbf{n}-\left(\mathcal{B}_{R} \rho_{k}\right) \mathbf{n}=\mathrm{e}^{i k t} \mathcal{F}_{\mathbb{T}}[\mathbf{H}](i k) & \text { on } S_{R} .
\end{array}
$$

Let $\mathbf{f}=\mathbf{F}_{S}, d=D_{S}, g=G_{S}, \mathbf{g}=\mathbf{G}_{S}$ and $\mathbf{h}=\mathbf{H}_{S}$ in equations (4.19), and let $\mathbf{v}, \mathfrak{p}$ and $\rho$ be unique solutions of equations (4.19). We write $\mathbf{u}_{S}=\mathbf{v}, \mathfrak{p}_{S}=\mathfrak{p}$ and $\rho_{S}=\rho$. Under these preparations, we set

$$
\begin{aligned}
& \mathbf{u}=\mathbf{u}_{S}+\sum_{1 \leq|k| \leq k_{0}+3} \mathbf{u}_{k}+\mathbf{u}_{\psi}, \\
& \mathfrak{p}=\mathfrak{p}_{S}+\sum_{1 \leq|k| \leq k_{0}+3} \mathfrak{p}_{k}+\mathfrak{p}_{\psi}, \\
& \rho=\rho_{S}+\sum_{1 \leq|k| \leq k_{0}+3} \rho_{k}+\rho_{\psi}
\end{aligned}
$$

and then $\mathbf{u}, \mathfrak{p}$ and $\rho$ are unique solutions of equations (4.1). Moreover, by Theorem 14, Theorem 15, and Theorem 16, we see that $\mathbf{u}, \mathfrak{p}$ and $\rho$ satisfy the estimate (4.2). In fact, for $f=f_{S}+\sum_{1 \leq|k| \leq k_{0}+3} \mathrm{e}^{i k t} f_{k}+f_{\psi}$, we have the following estimates:

$$
\begin{aligned}
\|f\|_{L_{p}((0,2 \pi), X)} & \leq\left\|f_{S}\right\|_{L_{p}((0,2 \pi), X)}+\sum_{1 \leq|k| \leq k_{0}+3}\left\|\mathrm{e}^{i k t} f_{k}\right\|_{L_{p}((0,2 \pi), X)}+\left\|f_{\psi}\right\|_{L_{p}((0,2 \pi), X)} \\
& \leq(2 \pi)^{1 / p}\left\|f_{S}\right\|_{X}+(2 \pi)^{1 / p} \sum_{1 \leq|k| \leq k_{0}+3}\left\|f_{k}\right\|_{X}+\left\|f_{\psi}\right\|_{L_{p}((0,2 \pi), X)}, \\
\left\|\partial_{t} f\right\|_{L_{p}((0,2 \pi), X)} & \leq \sum_{1 \leq|k| \leq k_{0}+3}\left\|(i k) \mathrm{e}^{i k t} f_{k}\right\|_{L_{p}((0,2 \pi), X)}+\left\|\partial_{t} f_{\psi}\right\|_{L_{p}((0,2 \pi), X)} \\
& \leq(2 \pi)^{1 / p}\left(k_{0}+3\right) \sum_{1 \leq|k| \leq k_{0}+3}\left\|f_{k}\right\|_{X}+\left\|\partial_{t} f_{\psi}\right\|_{L_{p}((0,2 \pi), X) .}
\end{aligned}
$$

By Hölder's inequality, we have

$$
\left\|f_{S}\right\|_{L_{p}((0,2 \pi), X)} \leq 2 \pi\|f\|_{L_{p}((0,2 \pi), X)},
$$




$$
\left\|\mathrm{e}^{i k t} \mathcal{F}_{\mathbb{T}}[f](i k)\right\|_{L_{p}((0,2 \pi), X)} \leq 2 \pi\|f\|_{L_{p}((0,2 \pi), X)},
$$

and for any UMD Banach space $X$, using Lemma 13 and transference theorem, Theorem 9, we have

$$
\begin{aligned}
\left\|f_{\psi}\right\|_{L_{p}((0,2 \pi), X)}, \leq & C\|\psi\|_{H_{\infty}^{1}}\|f\|_{L_{p}((0,2 \pi), X)}, \\
\left\|\partial_{t} f_{\psi}\right\|_{L_{p}((0,2 \pi), X)} \leq & C\|\psi\|_{H_{\infty}^{1}}\left\|\partial_{t} f\right\|_{L_{p}((0,2 \pi), X)}, \\
\left\|\Lambda^{1 / 2} f_{\psi}\right\|_{L_{p}((0,2 \pi), X) \leq} \leq \| \mathcal{F}_{\mathbb{T}}^{-1}\left[\left((i k)^{1 / 2} /\left(1+k^{2}\right)^{1 / 4}\right) \psi(i k)\left(1+k^{2}\right)^{1 / 4}\right. & \left.\mathcal{F}_{\mathbb{T}}[f](i k)\right] \|_{L_{p}((0,2 \pi), X)} \\
\leq & C\left(\sum_{\ell=0,1} \sup _{\lambda \in \mathbb{R}}\left|\left(\lambda \frac{d}{\mathrm{~d} \lambda}\right)^{\ell}\left(\left((i \lambda)^{1 / 2} /\left(1+\lambda^{2}\right)^{1 / 4}\right) \psi(i \lambda)\right)\right|\right) \\
& \|f\|_{H_{p}^{1 / 2}((0,2 \pi), X)} \cdot
\end{aligned}
$$

4.2. On linearized problem of two-phase problem

In this subsection, we consider the linear equations:

$$
\begin{aligned}
\partial_{t} \mathbf{u}_{ \pm}-\operatorname{Div}\left(\mu \mathbf{D}\left(\mathbf{u}_{ \pm}\right)-\mathfrak{p}_{ \pm} \mathbf{I}\right)=\mathbf{F}_{ \pm} & \text {in } \Omega_{ \pm} \times(0,2 \pi), \\
\operatorname{div} \mathbf{u}_{ \pm}=G_{ \pm}=\operatorname{div} \mathbf{G}_{ \pm} & \text {in } \Omega_{ \pm} \times(0,2 \pi), \\
\partial_{t} \rho+\mathcal{M} \rho-(\mathcal{A} \mathbf{u}) \cdot \mathbf{n}=D & \text { on } S_{R} \times(0,2 \pi), \\
{[[\mu \mathbf{D}(\mathbf{u})-\mathfrak{p} \mathbf{I})]] \mathbf{n}-\left(\mathcal{B}_{R} \rho\right) \mathbf{n}=\mathbf{H} } & \text { on } S_{R} \times(0,2 \pi), \\
{[[\mathbf{u}]]=0 } & \text { on } S_{R} \times(0,2 \pi), \\
\mathbf{u}_{-}=0 & \text { on } S \times(0,2 \pi) .
\end{aligned}
$$

where $\Omega_{+}=B_{R}, \Omega_{-}=\Omega \backslash\left(B_{R} \cup S_{R}\right)$, and $\mathcal{M}, \mathcal{A}$ and $\mathcal{B}_{R}$ are the linear operators defined in (2.17). We shall prove the unique existence theorem of $2 \pi$-periodic solutions of equations (4.29). Our main result in this section is stated as follows.

Theorem 17. Let $1<p, q<\infty$. Then, for any $\mathbf{F}_{ \pm}, D, G_{ \pm}, \mathbf{G}_{ \pm}$and $\mathbf{H}$ with

$$
\begin{aligned}
\mathbf{F}_{ \pm} \in L_{p, \operatorname{per}}\left((0,2 \pi), L_{q}\left(\Omega_{ \pm}\right)^{N}\right), \quad D \in L_{p, \operatorname{per}}\left((0,2 \pi), W_{q}^{2-1 / q}\left(S_{R}\right)\right) \\
G_{ \pm} \in L_{p, \operatorname{per}}\left((0,2 \pi), H_{q}^{1}\left(\Omega_{ \pm}\right)\right) \cap H_{p, \operatorname{per}}^{1 / 2}\left((0,2 \pi), L_{q}\left(\Omega_{ \pm}\right)\right) \\
\quad \mathbf{G}_{ \pm} \in H_{p, \operatorname{per}}^{1}\left((0,2 \pi), L_{q}\left(\Omega_{ \pm}\right)^{N}\right) \\
\mathbf{H} \in L_{p, \operatorname{per}}\left((0,2 \pi), H_{q}^{1}(\Omega)^{N}\right) \cap H_{p, \operatorname{per}}^{1 / 2}\left((0,2 \pi), L_{q}(\Omega)^{N}\right),
\end{aligned}
$$

problem (4.1) admits unique solutions $\mathbf{u}_{ \pm}, \mathfrak{p}_{ \pm}$and $\rho$ with

$$
\begin{aligned}
& \mathbf{u}_{ \pm} \in L_{p, \operatorname{per}}\left((0,2 \pi), H_{q}^{2}\left(\Omega_{ \pm}\right)^{N}\right) \cap H_{p, \operatorname{per}}^{1}\left((0,2 \pi), L_{q}\left(\Omega_{ \pm}\right)^{N}\right), \\
& \mathfrak{p}_{ \pm} \in L_{p, \operatorname{per}}\left((0,2 \pi), H_{q}^{1}\left(\Omega_{ \pm}\right)\right), \quad \sum_{ \pm} \int_{\Omega_{ \pm}} \mathfrak{p}_{ \pm}(x, t) \mathrm{d} x=0 \text { for } t \in(0,2 \pi),
\end{aligned}
$$




$$
\rho \in L_{p, \operatorname{per}}\left((0,2 \pi), W_{q}^{3-1 / q}\left(S_{R}\right)\right) \cap H_{p, \operatorname{per}}^{1}\left((0,2 \pi), W_{q}^{2-1 / q}\left(S_{R}\right)\right)
$$

possessing the estimate:

$$
\begin{aligned}
& \sum_{ \pm}\left\{\left\|\mathbf{u}_{ \pm}\right\|_{L_{p}\left((0,2 \pi), H_{q}^{2}\left(\Omega_{ \pm}\right)\right)}+\left\|\partial_{t} \mathbf{u}_{ \pm}\right\|_{L_{p}\left((0,2 \pi), L_{q}\left(\Omega_{ \pm}\right)\right)}+\left\|\nabla \mathfrak{p}_{ \pm}\right\|_{L_{p}\left((0,2 \pi), L_{q}\left(\Omega_{ \pm}\right)\right)}\right\} \\
& \quad+\|\rho\|_{L_{p}\left((0,2 \pi), W_{q}^{3-1 / q}\left(S_{R}\right)\right)}+\left\|\partial_{t} \rho\right\|_{L_{p}\left((0,2 \pi), W_{q}^{2-1 / q}\left(S_{R}\right)\right)} \\
& \leq C\left\{\sum_{ \pm}\left\|\mathbf{F}_{ \pm}\right\|_{L_{p}\left((0,2 \pi), L_{q}\left(\Omega_{ \pm}\right)\right)}+\|D\|_{L_{p}\left((0,2 \pi), W_{q}^{2-1 / q}\left(S_{R}\right)\right)}\right. \\
& \quad+\sum_{ \pm}\left\|\partial_{t} \mathbf{G}_{ \pm}\right\|_{L_{p}\left((0,2 \pi), L_{q}\left(\Omega_{ \pm}\right)\right)} \\
& \quad+\sum_{ \pm}\left\|G_{ \pm}\right\|_{L_{p}\left((0,2 \pi), H_{q}^{1}\left(\Omega_{ \pm}\right)\right)}+\left\|G_{ \pm}\right\|_{H_{p}^{1 / 2}\left((0,2 \pi), L_{q}\left(\Omega_{ \pm}\right)\right)} \\
& \left.\quad+\|\mathbf{H}\|_{L_{p}\left((0,2 \pi), H_{q}^{1}(\Omega)\right)}+\|\mathbf{H}\|_{H_{p}^{1 / 2}\left((0,2 \pi), L_{q}(\Omega)\right)}\right\}
\end{aligned}
$$

for some constant $C>0$.

To prove Theorem 17, the strategy is the same as in the proof of Theorem 6 . Therefore, we first consider the $\mathcal{R}$-solver of the generalized resolvent problem:

$$
\begin{aligned}
i k \mathbf{v}_{ \pm}-\operatorname{Div}\left(\mu \mathbf{D}\left(\mathbf{v}_{ \pm}\right)-\mathfrak{q}_{ \pm} \mathbf{I}\right)=\mathbf{f}_{ \pm} & \text {in } \Omega_{ \pm}, \\
\operatorname{div} \mathbf{v}_{ \pm}=g_{ \pm}=\operatorname{div} \mathbf{g}_{ \pm} & \text {in } \Omega_{ \pm}, \\
i k \eta+\mathcal{M} \eta-\left(\mathcal{A} \mathbf{v}_{+}\right) \cdot \mathbf{n}=d & \text { on } S_{R}, \\
{[[\mu \mathbf{D}(\mathbf{v})-\mathfrak{q} \mathbf{I}]] \mathbf{n}-\left(\mathcal{B}_{R} \eta\right) \mathbf{n}=\mathbf{h} } & \text { on } S_{R}, \\
{[[\mathbf{v}]]=0 } & \text { on } S_{R}, \\
\mathbf{v}_{-}=0 & \text { on } S
\end{aligned}
$$

for $k \in \mathbb{R}$. From Theorem 2.1.4 in Shibata and Saito [19] we know the following theorem concerned with the existence of an $\mathcal{R}$-solver of problem (4.29).

Theorem 18. Let $1<q<\infty$ and let $\mathbb{R}_{k_{0}}=\mathbb{R} \backslash\left(-k_{0}, k_{0}\right)$. Let

$$
\begin{aligned}
& X_{q}(\dot{\Omega})=\left\{(\mathbf{f}, d, \mathbf{h}, g, \mathbf{g}) \mid \mathbf{f} \in L_{q}(\dot{\Omega}), d \in W_{q}^{2-1 / q}\left(S_{R}\right),\right. \\
&\left.\mathbf{h} \in H_{q}^{1}(\Omega)^{N}, g \in H_{q}^{1}(\dot{\Omega}), \mathbf{g} \in L_{q}(\dot{\Omega})^{N}\right\}, \\
& \mathcal{X}_{q}(\dot{\Omega})=\left\{F=\left(F_{1}, F_{2}, \ldots, F_{7}\right) \mid F_{1}, F_{7} \in L_{q}(\dot{\Omega})^{N}, F_{2} \in W_{q}^{2-1 / q}\left(S_{R}\right),\right. \\
& F_{3} \in L_{q}(\Omega)^{N}, F_{4} \in H_{q}^{1}(\Omega)^{N}, \\
&\left.F_{5} \in L_{q}(\dot{\Omega}), \quad F_{6} \in H_{q}^{1}(\dot{\Omega})\right\} .
\end{aligned}
$$

Then, there exist a constant $k_{0}>0$ and operator families $\mathcal{A}(i k), \mathcal{P}(i k)$, and $\mathcal{H}(i k)$ with

$$
\mathcal{A}(i k) \in C^{1}\left(\mathbb{R}_{k_{0}}, \mathcal{L}\left(\mathcal{X}_{q}(\dot{\Omega}), H_{q}^{2}(\dot{\Omega})^{N}\right)\right)
$$




$$
\begin{aligned}
& \mathcal{P}(i k) \in C^{1}\left(\mathbb{R}_{k_{0}}, \mathcal{L}\left(\mathcal{X}_{q}(\dot{\Omega}), \dot{H}_{q}^{1}(\dot{\Omega})\right)\right), \\
& \mathcal{H}(i k) \in C^{1}\left(\mathbb{R}_{k_{0}}, \mathcal{L}\left(\mathcal{X}_{q}(\dot{\Omega}), W_{q}^{3-1 / q}\left(S_{R}\right)\right)\right)
\end{aligned}
$$

such that for any $(\mathbf{f}, d, \mathbf{h}, g, \mathbf{g})$ and $k \in \mathbb{R}_{k_{0}}, \mathbf{v}=\mathcal{A}(i k) \mathcal{F}_{k}, \mathfrak{q}=\mathcal{P}(i k) \mathcal{F}_{k}$ and $\eta=\mathcal{H}(i k) \mathcal{F}_{k}$, where

$$
\mathcal{F}_{k}=\left(\mathbf{f}, d,(i k)^{1 / 2} \mathbf{h}, \mathbf{h},(i k)^{1 / 2} g, g, i k \mathbf{g}\right),
$$

are unique solutions of equations (4.31), and

$$
\begin{array}{r}
\mathcal{R}_{\mathcal{L}\left(\mathcal{X}_{q}(\dot{\Omega}), H_{q}^{2-m}(\dot{\Omega})^{N}\right)}\left(\left\{\left(k \partial_{k}\right)^{\ell}\left((i k)^{m / 2} \mathcal{A}(i k)\right) \mid k \in \mathbb{R}_{k_{0}}\right\}\right) \leq r_{b}, \\
\mathcal{R}_{\mathcal{L}\left(\mathcal{X}_{q}(\dot{\Omega}), L_{q}(\dot{\Omega})^{N}\right)}\left(\left\{\left(k \partial_{k}\right)^{\ell} \nabla \mathcal{P}(i k) \mid k \in \mathbb{R}_{k_{0}}\right\}\right) \leq r_{b}, \\
\mathcal{R}_{\mathcal{L}\left(\mathcal{X}_{q}(\dot{\Omega}), W_{q}^{3-n-1 / q}\left(S_{R}\right)\right)}\left(\left\{\left(k \partial_{k}\right)^{\ell}\left((i k)^{n} \mathcal{H}(i k)\right) \mid k \in \mathbb{R}_{k_{0}}\right\}\right) \leq r_{b}
\end{array}
$$

for $\ell=0,1, m=0,1,2$ and $n=0,1$ with some constant $r_{b}$.

Remark 19. (1) Here $f \in L_{q}(\dot{\Omega})$ means that $f_{ \pm} \in L_{q}\left(\Omega_{ \pm}\right)$, and $f \in H_{q}^{1}(\dot{\Omega})$ means that $f_{ \pm} \in H_{q}^{1}\left(\Omega_{ \pm}\right)$, and we set

$$
\|f\|_{L_{q}(\dot{\Omega})}=\sum_{ \pm}\left\|f_{ \pm}\right\|_{L_{q}\left(\Omega_{ \pm}\right)}, \quad\|f\|_{H_{q}^{1}(\dot{\Omega})}=\sum_{ \pm}\left\|f_{ \pm}\right\|_{H_{q}^{1}\left(\Omega_{ \pm}\right)} .
$$

Moreover, we define

$$
\dot{H}_{q}^{1}(\dot{\Omega})=\left\{\theta \in H_{q}^{1}(\dot{\Omega}) \mid \int_{\dot{\Omega}} \theta \mathrm{d} x=0\right\} .
$$

(2) For $f$ defined on $\dot{\Omega}$, we set $f_{ \pm}=\left.f\right|_{\Omega_{ \pm}}$and for $f_{ \pm}$defined on $\Omega_{ \pm}$, we set $f=f_{ \pm}$ on $\Omega_{ \pm}$. The functions $F_{1}, F_{2}, F_{3}, F_{4}, F_{5}, F_{6}$, and $F_{7}$ are variables corresponding to $\mathbf{f}, d,(i k)^{1 / 2} \mathbf{h}, \mathbf{h},(i k)^{1 / 2} g, g$, and $i k \mathbf{g}$, respectively.

(3) We define the norm $\|\cdot\|_{\mathcal{X}_{q}(\Omega)}$ by setting

$$
\begin{aligned}
\left\|\left(F_{1}, \ldots, F_{7}\right)\right\|_{\mathcal{X}_{q}(\Omega)}= & \left\|\left(F_{1}, F_{5}, F_{7}\right)\right\|_{L_{q}(\dot{\Omega})}+\left\|F_{2}\right\|_{W_{q}^{2-1 / q}\left(S_{R}\right)}+\left\|F_{6}\right\|_{H_{q}^{1}(\dot{\Omega})} \\
& +\left\|F_{3}\right\|_{L_{q}(\Omega)}+\left\|F_{4}\right\|_{\left.H_{q}^{1}(\Omega)\right)} .
\end{aligned}
$$

Let $\varphi(i k)$ be a function in $C^{\infty}(\mathbb{R})$ which equals one for $k \in \mathbb{R}_{k_{0}+2}$ and zero for $k \notin \mathbb{R}_{k_{0}+1}$, and let $\psi(i k)$ be a function in $C^{\infty}(\mathbb{R})$ which equals one for $k \in \mathbb{R}_{k_{0}+4}$ and zero for $k \notin \mathbb{R}_{k_{0}+3}$. For $f \in\left\{\mathbf{F}_{ \pm}, G_{ \pm}, \mathbf{G}_{ \pm}, D, \mathbf{H}\right\}$, we set

$$
f_{\psi}=\mathcal{F}_{\mathbb{T}}^{-1}\left[\psi \mathcal{F}_{\mathbb{T}}[f]\right] .
$$

We consider the high frequency part of the equations (4.29):

$$
\begin{aligned}
\partial_{t} \mathbf{u}_{ \pm \psi}-\operatorname{Div}\left(\mu \mathbf{D}\left(\mathbf{u}_{ \pm \psi}\right)-\mathfrak{p}_{ \pm \psi} \mathbf{I}\right)=\mathbf{F}_{ \pm \psi} & \text { in } \Omega_{ \pm} \times(0,2 \pi), \\
\operatorname{div} \mathbf{u}_{ \pm \psi}=G_{ \pm \psi}=\operatorname{div} \mathbf{G}_{ \pm \psi} & \text { in } \Omega_{ \pm} \times(0,2 \pi), \\
\partial_{t} \rho_{\psi}+\mathcal{M} \rho_{\psi}-\left(\mathcal{A} \mathbf{u}_{+\psi}\right) \cdot \mathbf{n}=D_{\psi} & \text { on } S_{R} \times(0,2 \pi), \\
{\left[\left[\mu \mathbf{D}\left(\mathbf{u}_{\psi}\right)-\mathfrak{p}_{\psi} \mathbf{I}\right) \mathbf{n}-\left(\mathcal{B}_{R} \rho_{\psi}\right) \mathbf{n}=\mathbf{H}_{\psi}\right.} & \text { on } S_{R} \times(0,2 \pi), \\
{\left[\left[\mathbf{u}_{\psi}\right]\right]=0 } & \text { on } S_{R} \times(0,2 \pi), \\
\mathbf{u}_{-\psi}=0 & \text { on } S \times(0,2 \pi),
\end{aligned}
$$


By Theorem 8, Theorem 9, and the analogue of (4.5) resulting from (4.35), we have immediately the following theorem.

Theorem 20. Let $1<p, q<\infty$. Then, for any functions $\mathbf{F}, G, \mathbf{G}, D$, and $\mathbf{H}$ with

$$
\begin{aligned}
& \mathbf{F} \in L_{p, \operatorname{per}}\left((0,2 \pi), L_{q}(\dot{\Omega})^{N}\right), \quad D \in L_{p, \operatorname{per}}\left((0,2 \pi), W_{q}^{2-1 / q}\left(S_{R}\right)\right), \\
& \mathbf{H} \in H_{p, \operatorname{per}}^{1 / 2}\left((0,2 \pi), L_{q}(\Omega)^{N}\right) \cap L_{p, \operatorname{per}}\left((0,2 \pi), H_{q}^{1}(\Omega)^{N}\right), \\
& G \in H_{p, \operatorname{per}}^{1 / 2}\left((0,2 \pi), L_{q}(\dot{\Omega})\right) \cap L_{p, \operatorname{per}}\left((0,2 \pi), H_{q}^{1}(\dot{\Omega})\right), \\
& \quad \mathbf{G} \in H_{p, \operatorname{per}}^{1}\left((0,2 \pi), L_{q}(\dot{\Omega})^{N}\right),
\end{aligned}
$$

We let

$$
\begin{aligned}
& \mathbf{u}_{\psi}=\mathcal{F}_{\mathbb{T}}^{-1}\left[\varphi(i k) \mathcal{A}(i k) \mathcal{F}_{k}\left(\mathbf{F}_{\psi}, D_{\psi}, \mathbf{H}_{\psi}, G_{\psi}, \mathbf{G}_{\psi}\right)\right](\cdot, t), \\
& \mathfrak{p}_{\psi}=\mathcal{F}_{\mathbb{T}}^{-1}\left[\varphi(i k) \mathcal{P}(i k) \mathcal{F}_{k}\left(\mathbf{F}_{\psi}, D_{\psi}, \mathbf{H}_{\psi}, G_{\psi}, \mathbf{G}_{\psi}\right)\right](\cdot, t), \\
& \rho_{\psi}=\mathcal{F}_{\mathbb{T}}^{-1}\left[\varphi(i k) \mathcal{A}(i k) \mathcal{F}_{k}\left(\mathbf{F}_{\psi}, D_{\psi}, \mathbf{H}_{\psi}, G_{\psi}, \mathbf{G}_{\psi}\right)\right](\cdot, t),
\end{aligned}
$$

where we have set

$$
\begin{aligned}
\mathcal{F}_{k}\left(\mathbf{F}_{\psi}, D_{\pi}, \mathbf{H}_{\psi}, G_{\psi}, \mathbf{G}_{\psi}\right)= & \psi(i k)\left(\mathcal{F}_{\mathbb{T}}[\mathbf{F}](i k), \mathcal{F}_{\mathbb{T}}[D](i k),\right. \\
& (i k)^{1 / 2} \mathcal{F}_{\mathbb{T}}[\mathbf{H}](i k), \mathcal{F}_{\mathbb{T}}[\mathbf{H}](i k), \\
& \left.(i k)^{1 / 2} \mathcal{F}_{\mathbb{T}}[G](i k), \mathcal{F}_{\mathbb{T}}[G](i k), i k \mathcal{F}_{\mathbb{T}}[\mathbf{G}](i k)\right) .
\end{aligned}
$$

Then, $\mathbf{u}_{\psi}, \mathfrak{p}_{\psi}$ and $\rho_{\psi}$ are the unique solutions of equations (4.33), which possess the following estimate:

$$
\begin{aligned}
\| \mathbf{u}_{\psi} & \left\|_{L_{p}\left((0,2 \pi), H_{q}^{2}(\dot{\Omega})\right)}+\right\| \partial_{t} \mathbf{u}_{\psi}\left\|_{L_{p}\left((0,2 \pi), L_{q}(\dot{\Omega})\right)}+\right\| \nabla \mathfrak{p}_{\psi} \|_{L_{p}\left((0,2 \pi), L_{q}(\dot{\Omega})\right)} \\
& +\left\|\rho_{\psi}\right\|_{L_{p}\left((0,2 \pi), W_{q}^{3-1 / q}\left(S_{R}\right)\right)}+\left\|\partial_{t} \rho_{\psi}\right\|_{H_{p}^{1}\left((0,2 \pi), W_{q}^{2-1 / q}\left(S_{R}\right)\right)} \\
\leq & C\left\{\left\|\mathbf{F}_{\psi}\right\|_{L_{p}\left((0,2 \pi), L_{q}(\dot{\Omega})\right)}+\left\|D_{\psi}\right\|_{L_{p}\left((0,2 \pi), W_{q}^{2-1 / q}\left(S_{R}\right)\right)}+\left\|\partial_{t} \mathbf{G}_{\psi}\right\|_{L_{p}\left((0,2 \pi), L_{q}(\dot{\Omega})\right)}\right. \\
& +\left\|\Lambda^{1 / 2} G_{\psi}\right\|_{L_{p}\left((0,2 \pi), L_{q}(\dot{\Omega})\right)}+\left\|G_{\psi}\right\|_{L_{p}\left((0,2 \pi), H_{q}^{1}(\dot{\Omega})\right)} \\
& \left.+\left\|\Lambda^{1 / 2} \mathbf{H}_{\psi}\right\|_{L_{p}\left((0,2 \pi), L_{q}(\Omega)\right)}+\left\|\mathbf{H}_{\psi}\right\|_{L_{p}\left((0,2 \pi), H_{q}^{1}(\Omega)\right)}\right\}
\end{aligned}
$$

for some constant $C>0$. Here, we have set

$$
\Lambda^{1 / 2}\left(G_{\psi}, \mathbf{H}_{\psi}\right)=\mathcal{F}_{\mathbb{T}}^{-1}\left[(i k)^{1 / 2} \psi(i k)\left(\mathcal{F}_{\mathbb{T}}[G](i k), \mathcal{F}_{\mathbb{T}}[\mathbf{H}](i k)\right)\right]
$$

We now consider the lower frequency part of solutions of equations (4.29). Namely, we consider equations (4.31) for $k \in \mathbb{R}$ with $1 \leq|k|<k_{0}+4$. We shall show the following theorem.

Theorem 21. Let $1<q<\infty$ and $k \in \mathbb{Z}$ with $|k| \leq k_{0}+3$. Then, for any $\mathbf{f}_{ \pm} \in L_{q}\left(\Omega_{ \pm}\right)^{N}, g_{ \pm} \in H_{q}^{1}\left(\Omega_{ \pm}\right), d \in W_{q}^{2-1 / q}\left(S_{R}\right), \mathbf{h} \in H_{q}^{1}(\Omega)^{N}$, and $\mathbf{g}_{ \pm} \in$ 
$L_{q}\left(\Omega_{ \pm}\right)^{N}$, problem (4.31) admits unique solutions $\mathbf{v}_{ \pm} \in H_{q}^{2}\left(\Omega_{ \pm}\right)^{N}, \mathfrak{q}_{ \pm} \in H_{q}^{1}\left(\Omega_{ \pm}\right)$ with $\int_{\Omega} \mathfrak{q} \mathrm{d} x=0$, and $\eta \in W_{q}^{3-1 / q}\left(S_{R}\right)$ possessing the estimate:

$$
\begin{aligned}
& \|\mathbf{v}\|_{H_{q}^{2}(\dot{\Omega})}+\|\nabla \mathfrak{q}\|_{L_{q}(\dot{\Omega})}+\|\eta\|_{W_{q}^{3-1 / q}\left(S_{R}\right)} \\
& \quad \leq C\left(\|\mathbf{f}\|_{L_{q}(\dot{\Omega})}+\|d\|_{W_{q}^{2-1 / q}\left(S_{R}\right)}+\|g\|_{H_{q}^{1}(\dot{\Omega})}+\|\mathbf{g}\|_{L_{q}(\dot{\Omega})}+\|\mathbf{h}\|_{H_{q}^{1}(\Omega)}\right)
\end{aligned}
$$

for some constant $C>0$.

Proof. The strategy of the proof is the same as that in Theorem 15. The only difference is the reduced problem. First, we can reduce equations (4.31) to equations:

$$
\begin{aligned}
i k \mathbf{v}-\operatorname{Div}(\mu \mathbf{D}(\mathbf{v})-\mathfrak{p} \mathbf{I})=\mathbf{f} & \text { in } \dot{\Omega}, \\
\operatorname{div} \mathbf{v}=0 & \text { in } \dot{\Omega}, \\
i k \rho+\mathcal{M} \rho-\left(\mathcal{A} \mathbf{v}_{+}\right) \cdot \mathbf{n}=d & \text { on } S_{R}, \\
{[[\mu \mathbf{D}(\mathbf{v})-\mathfrak{p} \mathbf{I}]] \mathbf{n}-\left(\mathcal{B}_{R} \rho\right) \mathbf{n}=0 } & \text { on } S_{R}, \\
{[[\mathbf{v}]]=0 } & \text { on } S_{R}, \\
\mathbf{v}_{-}=0 & \text { on } S .
\end{aligned}
$$

For any $\mathbf{v}_{ \pm} \in H_{q}^{2}\left(\Omega_{ \pm}\right)^{N}$ and $\rho \in W_{q}^{3-1 / q}\left(S_{R}\right)$, let $K=K(\mathbf{v}, \rho) \in \dot{H}_{q}^{1}(\dot{\Omega})$ be the unique solution of the weak Neumann problem:

$$
(\nabla K, \nabla \varphi)_{\dot{\Omega}}=(\operatorname{Div}(\mu \mathbf{D}(\mathbf{v}))-\nabla \operatorname{div} \mathbf{v}, \nabla \varphi)_{\dot{\Omega}} \quad \text { for any } \varphi \in \dot{H}_{q^{\prime}}^{1}(\Omega)
$$

subject to the transmission condition:

$$
[[K]]=<[[\mu \mathbf{D}(\mathbf{v})]] \mathbf{n}, \mathbf{n}>-\sigma\left(\mathcal{B}_{R} \zeta\right) \mathbf{n}-[[\operatorname{div} \mathbf{v}]] \quad \text { on } S_{R}
$$

where $\mu$ is piecewise constant defined by $\left.\mu\right|_{\Omega_{ \pm}}=\mu_{ \pm}$. Here and in the following, $\dot{H}_{q}^{1}(\Omega)$ is defined by setting

$$
\dot{H}_{q}^{1}(\Omega)=\left\{\varphi \in H_{q}^{1}(\Omega) \mid \int_{\Omega} \varphi \mathrm{d} x=0\right\} .
$$

The reduced problem corresponding to equations (4.35) is

$$
\begin{aligned}
& i k \mathbf{v}-\operatorname{Div}(\mu \mathbf{D}(\mathbf{v})-K(\mathbf{v}, \rho) \mathbf{I})=\mathbf{f} \quad \text { in } \dot{\Omega}, \\
& i k \rho+\mathcal{M} \rho-\left(\mathcal{A} \mathbf{v}_{+}\right) \cdot \mathbf{n}=d \quad \text { on } S_{R}, \\
& {[[\mu \mathbf{D}(\mathbf{v})-K(\mathbf{v}, \rho) \mathbf{I}]] \mathbf{n}-\sigma\left(\mathcal{B}_{R} \rho\right) \mathbf{n}=0 \quad \text { on } S_{R} \text {, }} \\
& {[[\mathbf{v}]]=0 \quad \text { on } S_{R} \text {, }} \\
& \mathbf{v}_{-}=0 \quad \text { on } S \text {. }
\end{aligned}
$$

Let $J_{q}(\dot{\Omega})$ be the solenoidal space defined by setting

$$
J_{q}(\dot{\Omega})=\left\{\mathbf{u} \in L_{q}(\dot{\Omega}) \mid(\mathbf{u}, \nabla \varphi)_{\dot{\Omega}}=0 \text { for any } \varphi \in \dot{H}_{q^{\prime}}^{1}(\Omega)\right\} .
$$


For any $\mathbf{f} \in J_{q}(\dot{\Omega})$ and $d \in W_{q}^{2-1 / q}\left(S_{R}\right)$, problems (4.35) and (4.38) are equivalent. In fact, if problem (4.35) admits unique solutions $\mathbf{v} \in H_{q}^{2}(\dot{\Omega})^{N}, \mathfrak{p} \in \dot{H}_{q}^{1}(\dot{\Omega})$ and $\rho \in$ $W_{q}^{3-1 / q}\left(S_{R}\right)$, then using the divergence theorem of Gauss and noting that $[[\varphi]]=0$ on $S_{R}$ gives that for any $\varphi \in \dot{H}_{q^{\prime}}^{1}(\Omega)$,

$$
\begin{aligned}
0= & (\mathbf{f}, \nabla \varphi)_{\dot{\Omega}}=i k(\mathbf{v}, \nabla \varphi)_{\dot{\Omega}}-(\nabla \operatorname{div} \mathbf{v}, \nabla \varphi)_{\dot{\Omega}} \\
& +(\nabla(\mathfrak{p}-K(\mathbf{v}, \rho)), \nabla \varphi)_{\dot{\Omega}}=(\nabla(\mathfrak{p}-K(\mathbf{v}, \rho)), \nabla \varphi)_{\dot{\Omega}}
\end{aligned}
$$

because $\operatorname{div} \mathbf{v}=0$ on $\dot{\Omega}$. Moreover, the transmission conditions in (4.35) and (4.37) gives that

$$
[[\mathfrak{p}-K(\mathbf{v}, \rho)]]=[[\operatorname{div} \mathbf{v}]]=0 \quad \text { on } S_{R} .
$$

Thus, the uniqueness of the weak Neumann problem in $\dot{H}_{q}^{1}(\dot{\Omega})$ yields that $\mathfrak{p}-$ $K(\mathbf{v}, \rho)=0$ in $\Omega$. Thus, $\mathbf{v}$ and $\rho$ satisfy the equations (4.38).

Conversely, if $\mathbf{v} \in H_{q}^{2}(\dot{\Omega})^{N}$ and $\rho \in W_{q}^{3-1 / q}\left(S_{R}\right)$ satisfy equations (4.38), then the divergence theorem of Gauss gives that for any $\varphi \in \dot{H}_{q^{\prime}}^{1}(\Omega)$ we have

$$
0=(\mathbf{f}, \nabla \varphi)_{\dot{\Omega}}=i k(\mathbf{v}, \nabla \varphi)_{\dot{\Omega}}-(\nabla \operatorname{div} \mathbf{v}, \nabla \varphi)_{\dot{\Omega}}=-\left\{i k(\operatorname{div} \mathbf{v}, \varphi)_{\dot{\Omega}}+(\nabla \operatorname{div} \mathbf{v}, \nabla \varphi)_{\dot{\Omega}}\right\} .
$$

Moreover, the transmission conditions in (4.38) and (4.37) give that

$$
[[\operatorname{div} \mathbf{v}]]=<[[\mu \mathbf{D}(\mathbf{v})]] \mathbf{n}, \mathbf{n}>-\sigma\left(\mathcal{B}_{R} \zeta\right)-[[K]]=0 \text { on } S_{R}
$$

Thus, the uniqueness of this weak Neumann problem yields that $\operatorname{div} \mathbf{v}=c$ in $\dot{\Omega}$ for some global constant $c$. Now the divergence theorem of Gauss and the boundary conditions in (4.38) yield $c=0$, that is, $\operatorname{div} \mathbf{v}=0$, which shows that $\mathbf{v}, \mathfrak{p}=K(\mathbf{v}, \rho)$ and $\rho$ satisfy equations (4.35).

Employing the same argument as that in the proof of Theorem 15, we see that to prove Theorem 21, it is sufficient to prove the uniqueness of solutions to equations (4.38) in the $L_{2}$ framework. Thus, we choose $\mathbf{v} \in H_{2}^{2}(\dot{\Omega})^{N}$ and $\rho \in W_{2}^{5 / 2}\left(S_{R}\right)$ be solutions of the homogeneous equations:

$$
\begin{aligned}
& i k \mathbf{v}-\operatorname{Div}(\mu \mathbf{D}(\mathbf{v})-K(\mathbf{v}, \rho) \mathbf{I})=0 \quad \text { in } \dot{\Omega}, \\
& i k \rho+\mathcal{M} \rho-\left(\mathcal{A} \mathbf{v}_{+}\right) \cdot \mathbf{n}=0 \quad \text { on } S_{R}, \\
& {[[\mu \mathbf{D}(\mathbf{v})-K(\mathbf{v}, \rho) \mathbf{I}]] \mathbf{n}-\sigma\left(\mathcal{B}_{R} \rho\right) \mathbf{n}=0 \quad \text { on } S_{R} \text {, }} \\
& \text { [[v] }]=0 \quad \text { on } S_{R} \text {, } \\
& \mathbf{v}_{-}=0 \quad \text { on } S \text {, }
\end{aligned}
$$

and we shall show that $\mathbf{v}=0$ and $\rho=0$. Notice that $\operatorname{div} \mathbf{v}=0$ on $\dot{\Omega}$. Moreover, by $[[\mathbf{v}]]=0$, we have $\mathbf{v} \in H_{q}^{1}(\Omega) \cap H_{q}^{2}(\dot{\Omega})$. Integrating the second equation in (4.39) over $S_{R}$ and using the divergence theorem of Gauss on $\Omega_{+}=B_{R}$ gives that

$$
0=i k(\rho, 1)_{S_{R}}+\int_{S_{R}} \rho \mathrm{d} \omega\left|S_{R}\right|
$$




$$
-\int_{B_{R}} \operatorname{div}\left(\mathbf{v}_{+}-\frac{1}{\left|B_{R}\right|} \int_{B_{R}} \mathbf{v}_{+} \mathrm{d} y\right) \mathrm{d} x=\left(i k+\left|S_{R}\right|\right) \int_{S_{R}} \rho \mathrm{d} \omega\left|S_{R}\right|
$$

because $\operatorname{div} \mathbf{v}_{+}=0$ on $B_{R}$, and so $(\rho, 1)_{S_{R}}=0$. Moreover, multiplying the second equation in (4.39) by $x_{j}$ and integrating over $S_{R}$, similar arguments lead to

$$
\begin{aligned}
0 & =i k\left(\rho, x_{j}\right)_{S_{R}}+\int_{S_{R}} \rho x_{j} \mathrm{~d} \omega\left(x_{j}, x_{j}\right)_{S_{R}}-\int_{B_{R}} \operatorname{div}\left\{x_{j}\left(\mathbf{v}_{+}(x)-\frac{1}{\left|B_{R}\right|} \int_{B_{R}} \mathbf{v}_{+} \mathrm{d} y\right)\right\} \mathrm{d} x \\
& =i k\left(\rho, x_{j}\right)_{S_{R}}+\int_{S_{R}} \rho x_{j} \mathrm{~d} \omega\left(x_{j}, x_{j}\right)_{S_{R}}-\int_{B_{R}}\left(v_{+j}(x)-\frac{1}{\left|B_{R}\right|} \int_{B_{R}} v_{+j} \mathrm{~d} y\right) \mathrm{d} x \\
& =i k\left(\rho, x_{j}\right)_{S_{R}}+\int_{S_{R}} \rho x_{j} \mathrm{~d} \omega\left(x_{j}, x_{j}\right)_{S_{R}}
\end{aligned}
$$

because $\left(1, x_{j}\right)_{S_{R}}=0$, and $\left(x_{k}, x_{j}\right)_{S_{R}}=0$ for $j \neq k$. Since $\left(x_{j}, x_{j}\right)_{S_{R}}=$ $\left(R^{2} / N\right)\left|S_{R}\right|>0$, we have $\left(\rho, x_{j}\right)=0$. Summing up, we have proved

$$
(\rho, 1)_{S_{R}}=0, \quad\left(\rho, x_{j}\right)_{S_{R}}=0 \quad(j=1 \ldots, N) .
$$

In particular, $\mathcal{M} \rho=0$.

We now prove that $\mathbf{v}=0$. Multiplying the first equation of (4.39) with $\mathbf{v}$ and integrating the resultant formula over $\dot{\Omega}$ and using the divergence theorem of Gauss gives that

$$
0=i k\|\mathbf{v}\|_{L_{2}(\dot{\Omega})}^{2}-\sigma\left(\mathcal{B}_{R} \rho, \mathbf{n} \cdot \mathbf{v}\right)_{S_{R}}+\frac{\mu}{2}\|\mathbf{D}(\mathbf{v})\|_{L_{2}(\dot{\Omega})}^{2},
$$

because $\operatorname{div} \mathbf{v}=0$ in $\dot{\Omega}$. By the second equation of (4.39) with $\mathcal{M} \rho=0$, we have

$$
\sigma\left(\mathcal{B}_{R} \rho, \mathbf{n} \cdot \mathbf{v}\right)_{S_{R}}=\sigma\left(\mathcal{B}_{R} \rho, i k \rho\right)_{S_{R}}+\sum_{k=1}^{N} \frac{1}{\left|B_{R}\right|} \int_{B_{R}} w_{j} \mathrm{~d} t\left(\mathcal{B}_{R} \rho, R^{-1} x_{j}\right)_{S_{R}}
$$

where we have used $\mathbf{n}=R^{-1} x=R^{-1}\left(x_{1}, \ldots, x_{N}\right)$ for $x \in S_{R}$. This also yields

$$
\left(\mathcal{B}_{R} \rho, x_{j}\right)_{S_{R}}=\left(\rho,\left(\Delta_{S_{R}}+\frac{N-1}{R^{2}}\right) x_{j}\right)_{S_{R}}=0 .
$$

Moreover, since $\rho$ satisfies (4.40), we know that

$$
-\left(\mathcal{B}_{R} \rho, \rho\right)_{S_{R}} \geq c\|\rho\|_{L_{2}\left(S_{R}\right)}^{2}
$$

for some positive constant $c$, and therefore we have $\mathbf{D}(\mathbf{v})=0$. Since $\mathbf{v} \in H_{q}^{1}(\Omega)$ and $\mathbf{v}=0$ on $S_{-}$, we have $\mathbf{v}=0$.

Finally, the first equation of (4.39) yields that $\nabla K(\mathbf{v}, \rho)=0$, which shows that $K(\mathbf{v}, \rho)$ is constant in $\dot{\Omega}$. Thus, $[[K(\mathbf{v}, \rho)]]$ is constant. Integrating the third equation of (4.39) yields that

$$
[[K(\mathbf{v}, \rho)]] \int_{S_{R}} \mathrm{~d} \omega=\sigma\left(\Delta_{S_{R}} \rho, 1\right)_{S_{R}}+\frac{N-1}{R^{2}}(\rho, 1)_{S_{R}}=0
$$

where we have used (4.40). In particular, $K(\mathbf{v}, \rho)$ is a constant globally in $\Omega$. Finally, we have $\mathcal{B}_{R} \rho=0$ on $S_{R}$, which, combined with (4.40) leads to $\rho=0$. This completes the proof of uniqueness for equations (4.38) in the $L_{2}$ framework. Therefore, we have proved Theorem 21 . 
Proof of Theorem 17. Employing the same argument as in the proof of Theorem 6 and using Theorem 20 and Theorem 21, we can prove Theorem 17. We may omit the detailed proof.

\section{Proofs of main results}

In this section, we shall prove Theorem 4. The proof of Theorem 5 is parallel to that of Theorem 4, and so we may omit it. We prove Theorem 4 with the help of the usual Banach fixed-point argument, and we define an underlying space $\mathcal{I}_{\epsilon}$ with some small constant $\epsilon>0$ determined later by setting

$$
\begin{aligned}
& \mathcal{I}_{\epsilon}=\left\{(\mathbf{v}, h) \mid \quad \mathbf{v} \in L_{p, \operatorname{per}}\left((0,2 \pi), H_{q}^{2}\left(B_{R}\right)^{N}\right) \cap H_{p, \text { per }}^{1}\left((0,2 \pi), L_{q}\left(B_{R}\right)^{N}\right),\right. \\
& \quad h \in L_{p, \operatorname{per}}\left((0,2 \pi), W_{q}^{3-1 / q}\left(S_{R}\right)\right) \cap H_{p, \text { per }}^{1}\left((0,2 \pi), W_{q}^{2-1 / q}\left(S_{R}\right)\right) \cap H_{\infty, \text { per }}^{1}((0,2 \pi), \\
& \left.W_{q}^{1-1 / q}\left(S_{R}\right)\right), \\
& \left.\sup _{t \in(0,2 \pi)}\left\|H_{h}(\cdot, t)\right\|_{H_{\infty}^{1}\left(B_{R}\right)} \leq \delta, \quad E(\mathbf{v}, h) \leq \epsilon\right\},
\end{aligned}
$$

where we have set

$$
\begin{aligned}
E(\mathbf{v}, h)= & \|\mathbf{v}\|_{L_{p}\left((0,2 \pi), H_{q}^{2}\left(B_{R}\right)\right)}+\|\mathbf{v}\|_{H_{p}^{1}\left((0,2 \pi), L_{q}^{2}\left(B_{R}\right)\right)} \\
& +\|h\|_{L_{p}\left((0,2 \pi), W_{q}^{3-1 / q}\left(B_{R}\right)\right)}+\|h\|_{H_{p}^{1}\left((0,2 \pi), W_{q}^{2-1 / q}\left(B_{R}\right)\right)} \\
& +\left\|\partial_{t} h\right\|_{L_{\infty}\left((0,2 \pi), W_{q}^{1-1 / q}\left(S_{R}\right)\right)} .
\end{aligned}
$$

In view of (2.9), we define $\xi(t)$ by setting

$$
\xi(t)=\int_{0}^{t} \xi^{\prime}(s) \mathrm{d} s+c=\frac{1}{\left|B_{R}\right|} \int_{0}^{t} \int_{B_{R}} \mathbf{v}(x, s)\left(1+J_{0}(x, s)\right) \mathrm{d} x \mathrm{~d} s+c
$$

where $c$ is a constant for which

$$
\begin{aligned}
& \int_{0}^{2 \pi} \xi(s) \mathrm{d} s=0, \quad \text { that is, } \\
& c=-\frac{1}{2 \pi\left|B_{R}\right|} \int_{0}^{2 \pi}\left(\int_{0}^{t} \int_{B_{R}}\left(\mathbf{v}(x, s)\left(1+J_{0}(x, s)\right) \mathrm{d} x \mathrm{~d} s\right) \mathrm{d} t .\right.
\end{aligned}
$$

We choose $\delta>0$ so small that the map $x=\Phi(y, t)=y+\Psi(y, t)$ with $\Psi(y, t)=$ $\Psi_{h}(y, t)=R^{-1} H_{h}(y, t) y+\xi(t)$ is one to one. In particular, we may assume that $\delta>0$ and the inverse map: $y=\Xi(y, t)$ is well-defined and has the same regularity property as $\Phi(y, t)$. In particular, we may assume that

$$
\Xi(D) \subset B_{R}
$$

Since $\epsilon>0$ will be chosen small eventually, we may assume that $0<\epsilon<1$, and so for example, we estimate $\epsilon^{2}<\epsilon$ if necessary. Let $(\mathbf{v}, h) \in \mathcal{I}_{\epsilon}$ and let $\mathbf{u}$ and $\rho$ be 
solutions of linearized equations:

$$
\begin{cases}\partial_{t} \mathbf{u}+\mathcal{L} \mathbf{u}_{S}-\operatorname{Div}(\mu(\mathbf{D}(\mathbf{u})-\mathfrak{p} \mathbf{I})=\mathbf{G}+\mathbf{F}(\mathbf{v}, h) & \text { in } B_{R} \times(0,2 \pi), \\ \operatorname{div} \mathbf{u}=g(\mathbf{v}, h)=\operatorname{div} \mathbf{g}(\mathbf{v}, h) & \text { in } B_{R} \times(0,2 \pi), \\ \partial_{t} \rho+\mathcal{M} \rho-\mathcal{A} \mathbf{u} \cdot \mathbf{n}=\tilde{d}(\mathbf{v}, h) & \text { on } S_{R} \times(0,2 \pi), \\ (\mu \mathbf{D}(\mathbf{u})-\mathfrak{p} \mathbf{I}) \mathbf{n}-\left(\mathcal{B}_{R} \rho\right) \mathbf{n}=\mathbf{h}(\mathbf{v}, h) & \text { on } S_{R} \times(0,2 \pi) .\end{cases}
$$

In view of Theorem 6 , we shall show that

$$
\begin{aligned}
& \|\mathbf{F}(\mathbf{v}, h)\|_{L_{p}\left((0,2 \pi), L_{q}\left(B_{R}\right)\right)} \\
& \quad+\|\tilde{d}(\mathbf{v}, h)\|_{L_{p}\left((0,2 \pi), W_{q}^{2-1 / q}\left(S_{R}\right)\right)}+\|\left(g(\mathbf{v}, h), \mathbf{h}(\mathbf{v}, h) \|_{H_{p}^{1 / 2}\left((0,2 \pi), L_{q}\left(B_{R}\right)\right)}\right. \\
& \quad+\|\left(g(\mathbf{v}, h), \mathbf{h}(\mathbf{v}, h)\left\|_{L_{p}\left((0,2 \pi), H_{q}^{1}\left(B_{R}\right)\right)}+\right\| \partial_{t} \mathbf{g}(\mathbf{v}, h) \|_{L_{p}\left((0,2 \pi), L_{q}\left(B_{R}\right)\right)} \leq C \epsilon^{2},\right.
\end{aligned}
$$

for some constant $C>0$ independent of $\epsilon>0$. In the following, $C$ denotes generic constants independent of $\epsilon>0$, the value of which may change from line to line. Before starting with the estimates of the nonlinear terms, we summarize some inequalities which are useful for our estimations. The following inequalities follow from Sobolev's inequality and the estimate of the boundary trace:

$$
\begin{aligned}
\|f\|_{L_{\infty}\left(B_{R}\right)} & \leq C\|f\|_{H_{q}^{1}\left(B_{R}\right)}, \\
\|f g\|_{H_{q}^{1}\left(B_{R}\right)} & \leq C\|f\|_{H_{q}^{1}\left(B_{R}\right)}\|g\|_{H_{q}^{1}\left(B_{R}\right)}, \\
\|f g\|_{H_{q}^{2}\left(B_{R}\right)} & \leq C\left(\|f\|_{H_{q}^{2}\left(B_{R}\right)}\|g\|_{H_{q}^{1}\left(B_{R}\right)}+\|f\|_{H_{q}^{1}\left(B_{R}\right)}\|g\|_{H_{q}^{2}\left(B_{R}\right)}\right), \\
\|f g\|_{W_{q}^{1-1 / q}\left(S_{R}\right)} & \leq C\|f\|_{W_{q}^{1-1 / q}\left(S_{R}\right)}\|g\|_{W_{q}^{1-1 / q}\left(S_{R}\right)}, \\
\|f g\|_{W_{q}^{2-1 / q}\left(S_{R}\right)} & \leq C\left(\|f\|_{W_{q}^{2-1 / q}\left(S_{R}\right)}\|g\|_{W_{q}^{1-1 / q}\left(S_{R}\right)}+\|f\|_{W_{q}^{1-1 / q}\left(S_{R}\right)}\|g\|_{W_{q}^{2-1 / q}\left(S_{R}\right)}\right)
\end{aligned}
$$

for $N<q<\infty$ with some constant $C$. The following inequalities follow from real interpolation theorem and the periodicity of functions, which will be used to estimate the $L_{\infty}$ norm with respect to the time variable of lower order regularity terms with respect to the space variable $x$ :

$$
\begin{aligned}
\|\mathbf{v}\|_{L_{\infty}\left((0,2 \pi), B_{q, p}^{2(1-1 / q)}\left(B_{R}\right)\right)} & \leq C\left(\|\mathbf{v}\|_{L_{p}\left((0,2 \pi), H_{q}^{2}\left(B_{R}\right)\right)}+\left\|\partial_{t} \mathbf{v}\right\|_{L_{p}\left((0,2 \pi), L_{q}\left(B_{R}\right)\right)}\right), \\
\|h\|_{L_{\infty}\left((0,2 \pi), B_{q, p}^{3-1 / p-1 / q}\left(S_{R}\right)\right)} & \leq C\left(\|h\|_{L_{p}\left((0,2 \pi), W_{q}^{3-1 / q}\left(S_{R}\right)\right)}+\left\|\partial_{t} h\right\|_{L_{p}\left((0,2 \pi), W_{q}^{2-1 / q}\left(S_{R}\right)\right)}\right) .
\end{aligned}
$$

In fact, to obtain (5.8) we use the following well-known result: Let $X$ and $Y$ be two Banach spaces such that $Y$ is continuously embedded into $X$, and then $C\left([0, \infty),(X, Y)_{1-1 / p, p}\right)$ is continuously embedded into $H_{p}^{1}((0, \infty), X) \cap$ $L_{p}((0, \infty), Y)$ and

$$
\|f\|_{L_{\infty}\left((0, \infty),(X, Y)_{1-1 / p, p}\right)} \leq\|f\|_{L_{p}((0, \infty), Y)}+\|f\|_{H_{p}^{1}((0, \infty), X)} .
$$

For its proof, we refer to [9,21].

We start with the estimate of $\mathbf{F}(\mathbf{v}, h)$. From (3.11), we have

$$
\left\|\mathbf{F}_{1}(\mathbf{v}, h)\right\|_{L_{q}\left(B_{R}\right)} \leq C\left\{\|\mathbf{v}\|_{L_{\infty}\left(B_{R}\right)}\|\nabla \mathbf{v}\|_{L_{q}\left(B_{R}\right)}+\left\|\partial_{t} \Psi_{h}\right\|_{L_{\infty}\left(B_{R}\right)}\|\nabla \mathbf{v}\|_{L_{q}\left(B_{R}\right)}\right.
$$




$$
\begin{aligned}
& +\left\|\nabla \Psi_{h}\right\|_{L_{\infty}\left(B_{R}\right)}\left\|\partial_{t} \mathbf{v}\right\|_{L_{q}\left(B_{R}\right)}+\left\|\nabla \Psi_{h}\right\|_{L_{\infty}\left(B_{R}\right)}\left\|\nabla^{2} \mathbf{v}\right\|_{L_{q}\left(B_{R}\right)} \\
& \left.+\left\|\nabla^{2} \Psi_{h}\right\|_{L_{q}\left(B_{R}\right)}\|\nabla \mathbf{v}\|_{L_{\infty}\left(B_{R}\right)}\right) .
\end{aligned}
$$

By (5.7) and (2.5), we have

$$
\begin{aligned}
\left\|\mathbf{F}_{1}(\mathbf{v}, h)\right\|_{L_{p}\left((0,2 \pi), L_{q}\left(B_{R}\right)\right) \leq} & C\left\{\|\mathbf{v}\|_{L_{\infty}\left((0,2 \pi), H_{q}^{1}\left(B_{R}\right)\right)}\|\mathbf{v}\|_{L_{p}\left((0,2 \pi), H_{q}^{1}\left(B_{R}\right)\right)}\right. \\
& +\left\|\partial_{t} h\right\|_{L_{p}\left((0,2 \pi), W_{q}^{1-1 / q}\left(S_{R}\right)\right)}\|\mathbf{v}\|_{L_{\infty}\left((0,2 \pi), H_{q}^{1}\left(B_{R}\right)\right)} \\
& +\|h\|_{L_{\infty}\left((0,2 \pi), W_{q}^{2-1 / q}\left(S_{R}\right)\right)}\left(\left\|\partial_{t} \mathbf{v}\right\|_{L_{q}\left((0,2 \pi), L_{q}\left(B_{R}\right)\right)}\right. \\
& \left.+\|\mathbf{v}\|_{L_{p}\left((0,2 \pi), H_{q}^{2}\left(B_{R}\right)\right)}\right),
\end{aligned}
$$

which, combined with (5.8) and (5.1), leads to

$$
\left\|\mathbf{F}_{1}(\mathbf{v}, h)\right\|_{L_{p}\left((0,2 \pi), L_{q}\left(B_{R}\right)\right)} \leq C \epsilon^{2},
$$

because $1<2(1-1 / p)$ and $2-1 / q<3-1 / p-1 / q$. From (3.12), it follows that

$$
\begin{aligned}
& \left\|\mathbf{F}_{2}(\mathbf{v}, h)(\cdot, t)\right\|_{L_{q}\left(B_{R}\right)} \\
& \leq C \int_{0}^{2 \pi}\|\mathbf{v}(\cdot, t)\|_{L_{q}\left(B_{R}\right)}\left(\left\|J_{0}(\cdot, t)\right\|_{L_{\infty}\left(B_{R}\right)}\right. \\
& \left.\quad+\|\Psi(\cdot, t)\|_{L_{\infty}\left(B_{R}\right)}\left(1+\left\|J_{0}(\cdot, t)\right\|_{L_{\infty}\left(B_{R}\right)}\right)\right) \mathrm{d} t \\
& \quad+\int_{0}^{2 \pi}\|\mathbf{v}(\cdot, t)\|_{L_{q}\left(B_{R}\right)}\left(1+\|\Psi(\cdot, t)\|_{L_{\infty}\left(B_{R}\right)}\right)\left(1+\left\|J_{0}(\cdot, t)\right\|_{L_{\infty}\left(B_{R}\right)}\right) \mathrm{d} t\|\Psi(\cdot, t)\|_{L_{q}\left(B_{R}\right)} \\
& \quad+\|\nabla \Psi(\cdot, t)\|_{L_{q}\left(B_{R}\right)} \int_{0}^{2 \pi}\|\mathbf{v}(\cdot, t)\|_{L_{q}\left(B_{R}\right)}\left(1+\|\Psi(\cdot, t)\|_{L_{\infty}\left(B_{R}\right)}\right)\left(1+\left\|J_{0}(\cdot, t)\right\|_{L_{\infty}\left(B_{R}\right)}\right) \mathrm{d} y \mathrm{~d} t \\
& \quad \times\left(1+\|\Psi(\cdot, t)\|_{L_{\infty}\left(B_{R}\right)}\right) .
\end{aligned}
$$

To estimate $\mathbf{F}_{2}(\mathbf{v}, h)$, we recall

$$
J_{0}(y, t)=\operatorname{det}\left(\delta_{i j}+R^{-1} \frac{\partial}{\partial y_{j}} H_{h}(y, t) y_{i}\right)-1
$$

and that $\Psi(y, t)=R^{-1} H_{h}(y, t) y+\xi(t)$, where $\xi(t)$ is given by

$$
\begin{aligned}
\xi(t) & =\int_{0}^{t} \frac{1}{\left|B_{R}\right|} \int_{B_{R}}\left(\mathbf{v}(y, s)\left(1+J_{0}(y, s)\right) \mathrm{d} y \mathrm{~d} s+c,\right. \\
c & =-\int_{0}^{2 \pi} \int_{0}^{t} \frac{1}{\left|B_{R}\right|} \int_{B_{R}}\left(\mathbf{v}(y, s)\left(1+J_{0}(y, s)\right) \mathrm{d} y \mathrm{~d} s \mathrm{~d} t .\right.
\end{aligned}
$$

By (5.7) and (2.5) we obtain

$$
\begin{aligned}
\left\|H_{h}(\cdot, t)\right\|_{L_{\infty}\left(B_{R}\right)} & \leq C\|h(\cdot, t)\|_{W_{q}^{1-1 / q}\left(S_{R}\right)} \leq C \epsilon, \\
\left\|\nabla H_{h}(\cdot, t)\right\|_{L_{\infty}\left(B_{R}\right)} & \leq C\|h(\cdot, t)\|_{W_{q}^{2-1 / q}\left(S_{R}\right)} \leq C \epsilon,
\end{aligned}
$$

By (5.7), (2.5), (5.8), the fact that $2-1 / q<3-1 / p-1 / q$, and (5.1), we have

$$
\begin{aligned}
\left\|J_{0}(\cdot, t)\right\|_{L_{\infty}\left(B_{R}\right)} & \leq C\left\|\nabla H_{h}(\cdot, t)\right\|_{L_{\infty}\left(B_{R}\right)}\left(1+\left\|\nabla H_{h}(\cdot, t)\right\|_{L_{\infty}\left(B_{R}\right)}\right)^{N-1} \\
& \leq C\|h(\cdot, t)\|_{W_{q}^{2-1 / q}\left(S_{R}\right)}\left(1+\|h(\cdot, t)\|_{W_{q}^{2-1 / q}\left(S_{R}\right)}\right)^{N-1} \\
& \leq C \epsilon .
\end{aligned}
$$


From (5.10) and (5.1), it follows that

$$
|\xi(t)| \leq C\|\mathbf{v}\|_{L_{p}\left((0,2 \pi), L_{q}\left(B_{R}\right)\right)} \leq C \epsilon
$$

In particular, by (5.11) and (5.13), we have

$$
\|\Psi(\cdot, t)\|_{L_{\infty}\left(B_{R}\right)} \leq C \epsilon, \quad\|\nabla \Psi(\cdot, t)\|_{L_{\infty}\left(B_{R}\right)} \leq C \epsilon .
$$

Combining (5.1) and (5.14) gives that

$$
\left\|\mathbf{F}_{2}(\mathbf{v}, h)\right\|_{L_{p}\left((0,2 \pi), L_{q}\left(B_{R}\right)\right)} \leq C \epsilon\|\mathbf{v}\|_{L_{p}\left((0,2 \pi), L_{q}\left(B_{R}\right)\right)} \leq C \epsilon^{2},
$$

which, combined with (5.9), leads to

$$
\|\mathbf{F}(\mathbf{v}, h)\|_{L_{p}\left((0,2 \pi), L_{q}\left(B_{R}\right)\right)} \leq C \epsilon^{2} .
$$

By (5.4) and (5.14), we have

$$
\|\mathbf{G}\|_{L_{p}\left((0,2 \pi), L_{q}\left(B_{R}\right)\right)} \leq C\|\mathbf{f}\|_{L_{p}\left((0,2 \pi), L_{q}(D)\right)} .
$$

We next estimate $\tilde{d}(\mathbf{v}, h)$. By (3.25) and (5.1),

$$
\begin{aligned}
&\left\|\mathbf{n}_{t}-\mathbf{n}\right\|_{W_{q}^{1-1 / q}\left(S_{R}\right)} \leq C\left\|H_{h}(\cdot, t)\right\|_{H_{q}^{2}\left(B_{R}\right)} \leq C \epsilon \\
&\left\|\mathbf{n}_{t}-\mathbf{n}\right\|_{W_{q}^{2-1 / q}\left(S_{R}\right)} \leq C\left(\left\|H_{h}(\cdot, t)\right\|_{H_{q}^{3}\left(B_{R}\right)}+\left\|H_{h}(\cdot, t)\right\|_{H_{q}^{2}\left(B_{R}\right)}\left\|H_{h}(\cdot, t)\right\|_{H_{\infty}^{2}\left(B_{R}\right)}\right) .
\end{aligned}
$$

Since we assume that $2 / p+N / q<1$, we can choose $\kappa>0$ so small that $2+N / q+$ $\kappa-1 / q<3-1 / p-1 / q$ and $1+N / q+\kappa<2(1-1 / p)$, and then by Sobolev's inequality and (5.8) we have

$$
\begin{aligned}
& \sup _{t \in(0,2 \pi)}\|\mathbf{v}(\cdot, t)\|_{H_{\infty}^{1}\left(B_{R}\right)} \leq C \sup _{t \in(0,2 \pi)}\|\mathbf{v}(\cdot, t)\|_{B_{q, p}^{2(1-1 / p)}\left(B_{R}\right)} \leq C \epsilon ; \\
& \sup _{t \in(0,2 \pi)}\left\|H_{h}(\cdot, t)\right\|_{H_{\infty}^{2}\left(B_{R}\right)} \leq C \sup _{t \in(0,2 \pi)}\|h(\cdot, t)\|_{B_{q, p}^{3-1 / p-1 / q}\left(S_{R}\right)} \leq C \epsilon,
\end{aligned}
$$

where we have used (2.5) in the last inequality. Then, in particular, using again (2.5), we have

$$
\left\|\mathbf{n}_{t}-\mathbf{n}\right\|_{W_{q}^{2-1 / q}\left(S_{R}\right)} \leq C\left\|H_{h}(\cdot, t)\right\|_{H_{q}^{3}\left(B_{R}\right)} \leq C\|h(\cdot, t)\|_{W_{q}^{3-1 / q}\left(S_{R}\right)} .
$$

Thus, applying (5.12) to the formula in (2.11) and using (5.1) and (5.7) gives that

$$
\begin{aligned}
\|d(\mathbf{v}, h)\|_{L_{p}\left((0,2 \pi), W_{q}^{2-1 / q}\left(S_{R}\right)\right)} \leq & C \epsilon\left(\|\mathbf{v}\|_{L_{\infty}\left((0,2 \pi), H_{q}^{1}\left(B_{R}\right)\right)}+\|\mathbf{v}\|_{L_{p}\left((0,2 \pi), H_{q}^{2}\left(B_{R}\right)\right)}\right. \\
& +\left\|\partial_{t} h\right\|_{L_{\infty}\left((0,2 \pi), W_{q}^{1-1 / q}\left(S_{R}\right)\right)} \\
& \left.+\left\|\partial_{t} h\right\|_{L_{p}\left((0,2 \pi), W_{q}^{2-1 / q}\left(S_{R}\right)\right)}\right) \\
\leq & C \epsilon^{2} .
\end{aligned}
$$


On the other hand, by (5.11),

$$
\|h(\cdot, t)\|_{L_{\infty}\left(S_{R}\right)} \leq C\left\|H_{h}(\cdot, t)\right\|_{L_{\infty}\left(B_{R}\right)} \leq C \epsilon,
$$

and so

$$
\left|\int_{S_{R}} h^{k} \mathrm{~d} \omega\right| \leq C \epsilon^{2}, \quad\left|\int_{S_{R}} h^{k} \omega \mathrm{d} \omega\right| \leq C \epsilon^{2} \text { for } k \geq 2,
$$

which, combined with (5.18), leads to

$$
\|\tilde{d}(\mathbf{v}, h)\|_{L_{p}\left((0,2 \pi), W_{q}^{2-1 / q}\left(S_{R}\right)\right)} \leq C \epsilon^{2} .
$$

We next consider $\mathbf{g}(\mathbf{v}, h)$ given in (3.6), where $\rho$ is replaced by $h$. We may write

$$
\mathbf{g}(\mathbf{v}, h)=\mathbf{V}_{\mathbf{g}}(\mathbf{k})\left(H_{h}, \nabla H_{h}\right) \otimes \mathbf{v} .
$$

where $\mathbf{k}$ denotes variables corresponding to $\left(H_{h}, \nabla H_{h}\right)$ and $\mathbf{V}_{\mathbf{g}}$ is a $C^{\infty}$ function defined on $|\mathbf{k}|<\delta$. We write

$$
\begin{aligned}
\partial_{t} \mathbf{g}(\mathbf{v}, h)= & \mathbf{V}_{\mathbf{g}}^{\prime}(\mathbf{k}) \partial_{t}\left(H_{h}, \nabla H_{h}\right) \otimes\left(H_{h}, \nabla H_{h}\right) \otimes \mathbf{v}+\mathbf{V}_{\mathbf{g}}(\mathbf{k}) \partial_{t}\left(H_{h}, \nabla H_{h}\right) \otimes \mathbf{v} \\
& +\mathbf{V}_{\mathbf{g}}(\mathbf{k})\left(H_{h}, \nabla H_{h}\right) \otimes \partial_{t} \mathbf{v},
\end{aligned}
$$

and so, by (5.11), (2.5), we have

$$
\begin{aligned}
\left\|\partial_{t} \mathbf{g}(\mathbf{v}, h)\right\|_{L_{p}\left((0,2 \pi), L_{q}\left(B_{R}\right)\right) \leq} & C\left(\|\mathbf{v}\|_{L_{\infty}\left((0,2 \pi), H_{q}^{1}\left(B_{R}\right)\right)}+\|h\|_{L_{\infty}\left((0,2 \pi), W_{q}^{2-1 / q}\left(S_{R}\right)\right)}\right) \\
& \times\left(\|h\|_{H_{p}^{1}\left((0,2 \pi), W_{q}^{2-1 / q}\left(S_{R}\right)\right)}+\left\|\partial_{t} \mathbf{v}\right\|_{L_{p}\left((0,2 \pi), L_{q}\left(B_{R}\right)\right)}\right) \\
\leq & C \epsilon^{2} .
\end{aligned}
$$

We next estimate $g(\mathbf{v}, h)$ and $\mathbf{h}(\mathbf{v}, h)=\left(\mathbf{h}^{\prime}(\mathbf{v}, h), h_{N}(\mathbf{v}, h)\right)$ given in (3.6), (3.31) and (3.34), where $\rho$ is replaced by $h$. We may write

$$
g(\mathbf{v}, h)=V_{g}(\mathbf{k})\left(H_{h}, \nabla H_{h}\right) \otimes \nabla \mathbf{v},
$$

where $\mathbf{k}$ are variables corresponding to $\left(H_{h}, \nabla H_{h}\right)$ and $V_{g}(\mathbf{k})$ is some matrix of $C^{\infty}$ functions defined on $|\mathbf{k}|<\delta$. To estimate $g$, we use the following two lemmas.

Lemma 22. Let $1<p<\infty$ and $N<q<\infty$. Let

$$
\begin{aligned}
& f \in H_{\infty, \operatorname{per}}^{1}\left((0,2 \pi), L_{q}\left(B_{R}\right)\right) \cap L_{\infty, \operatorname{per}}\left((0,2 \pi), H_{q}^{1}\left(B_{R}\right)\right), \\
& g \in H_{p, \operatorname{per}}^{1 / 2}\left((0,2 \pi), L_{q}\left(B_{R}\right)\right) \cap L_{p, \operatorname{per}}\left((0,2 \pi), H_{q}^{1}\left(B_{R}\right)\right) .
\end{aligned}
$$

Then, we have

$$
\begin{aligned}
&\|f g\|_{H_{p}^{1 / 2}\left((0,2 \pi), L_{q}\left(B_{R}\right)\right)}+\|f g\|_{L_{p}\left((0,2 \pi), H_{q}^{1}\left(B_{R}\right)\right)} \\
& \leq C\left(\|f\|_{H_{\infty}^{1}\left((0,2 \pi), L_{q}\left(B_{R}\right)\right)}+\|f\|_{L_{\infty}\left((0,2 \pi), H_{q}^{1}\left(B_{R}\right)\right)}\right)^{1 / 2}\|f\|_{L_{\infty}\left((0,2 \pi), H_{q}^{1}\left(B_{R}\right)\right)}^{1 / 2} \\
& \quad \times\left(\|g\|_{H_{p}^{1 / 2}\left((0,2 \pi), L_{q}\left(B_{R}\right)\right)}+\|g\|_{L_{p}\left((0,2 \pi), H_{q}^{1}\left(B_{R}\right)\right)}\right)
\end{aligned}
$$

for some constant $C>0$. 
Proof. By (5.7), we have

$$
\|f g\|_{L_{p}\left((0,2 \pi), H_{q}^{1}\left(B_{R}\right)\right)} \leq\|f\|_{L_{\infty}\left((0,2 \pi), H_{q}^{1}\left(B_{R}\right)\right)}\|g\|_{L_{p}\left((0,2 \pi), H_{q}^{1}\left(B_{R}\right)\right)} .
$$

To estimate the $H^{1 / 2}$ norm, we use the complex interpolation relation:

$$
\begin{aligned}
& H_{p, \text { per }}^{1 / 2}\left((0,2 \pi), L_{q}\left(B_{R}\right)\right) \cap L_{p, \operatorname{per}}\left((0,2 \pi), H_{q}^{1 / 2}\left(B_{R}\right)\right) \\
& \quad=\left(L_{p, \operatorname{per}}\left((0,2 \pi), L_{q}\left(B_{R}\right)\right), H_{p, \operatorname{per}}^{1}\left((0,2 \pi), L_{q}\left(B_{R}\right)\right) \cap L_{p, \operatorname{per}}\left((0,2 \pi), H_{q}^{1}\left(B_{R}\right)\right)\right)_{1 / 2}
\end{aligned}
$$

where $(\cdot, \cdot)_{1 / 2}$ denotes a complex interpolation of order $1 / 2$. By $(5.7)$, we have

$$
\begin{aligned}
\|f g\|_{H_{p}^{1}\left((0,2 \pi), L_{q}\left(B_{R}\right)\right) \leq} & C\left(\left\|\partial_{t} f\right\|_{L_{\infty}\left((0,2 \pi), L_{q}\left(B_{R}\right)\right)}+\|f\|_{L_{\infty}\left((0,2 \pi), H_{q}^{1}\left(B_{R}\right)\right)}\right) \\
& \times\left(\|g\|_{L_{p}\left((0,2 \pi), H_{q}^{1}\left(B_{R}\right)\right)}+\left\|\partial_{t} g\right\|_{L_{p}\left((0,2 \pi), L_{q}\left(B_{R}\right)\right)}\right), \\
\|f g\|_{L_{p}\left((0,2 \pi), L_{q}\left(B_{R}\right)\right) \leq} \leq & C\|f\|_{L_{\infty}\left((0,2 \pi), H_{q}^{1}\left(B_{R}\right)\right)}\|g\|_{L_{p}\left((0,2 \pi), L_{q}\left(B_{R}\right)\right) .} .
\end{aligned}
$$

Thus, by (5.23), we have

$$
\begin{aligned}
\|f g\|_{H_{p}^{1 / 2}\left((0,2 \pi), L_{q}\left(B_{R}\right)\right)} \leq & C\left(\|f\|_{H_{\infty}^{1}\left((0,2 \pi), L_{q}\left(B_{R}\right)\right)}\right. \\
& \left.+\|f\|_{L_{\infty}\left((0,2 \pi), H_{q}^{1}\left(B_{R}\right)\right)}\right)^{1 / 2}\|f\|_{L_{\infty}\left((0,2 \pi), H_{q}^{1}\left(B_{R}\right)\right)}^{1 / 2} \\
& \times\left(\|g\|_{H_{p}^{1 / 2}\left((0,2 \pi), L_{q}\left(B_{R}\right)\right)}+\|g\|_{L_{p}\left((0,2 \pi), H_{q}^{1 / 2}\left(B_{R}\right)\right)}\right)
\end{aligned}
$$

Since $\|g\|_{L_{p}\left((0,2 \pi), H_{q}^{1 / 2}\left(B_{R}\right)\right)} \leq C\|g\|_{L_{p}\left((0,2 \pi), H_{q}^{1}\left(B_{R}\right)\right)}$, combining (5.22) and (5.24) leads to (5.21), which completes the proof of Lemma 22.

Lemma 23. Let $1<p, q<\infty$. Then, there exists a constant $C$ such that for any $u$ with

$$
u \in H_{p, \operatorname{per}}^{1}\left((0,2 \pi), L_{q}\left(B_{R}\right)\right) \cap L_{p, \operatorname{per}}\left((0,2 \pi), H_{q}^{2}\left(B_{R}\right)\right),
$$

we have

$$
\|u\|_{H_{p}^{1 / 2}\left((0,2 \pi), H_{q}^{1}\left(B_{R}\right)\right)} \leq C\left(\|u\|_{H_{p}^{1}\left((0,2 \pi), L_{q}\left(B_{R}\right)\right)}+\|u\|_{L_{p}\left((0,2 \pi), H_{q}^{2}\left(B_{R}\right)\right)}\right)
$$

for some constant $C>0$.

Proof. As was proved in the proof of Proposition 1 in Shibata [17], there exist two operators $\Phi_{1}$ and $\Phi_{2}$ with

$\Phi_{1} \in C^{1}\left(\mathbb{R} \backslash\{0\}, \mathcal{L}\left(L_{q}\left(B_{R}\right), L_{q}\left(B_{R}\right)^{N}\right)\right), \quad \Phi_{2} \in C^{1}\left(\mathbb{R} \backslash\{0\}, \mathcal{L}\left(H_{q}^{2}\left(B_{R}\right), L_{q}\left(B_{R}\right)^{N}\right)\right.$

such that for any $g \in H_{q}^{2}\left(B_{R}\right)$, we have

$$
\left(1+\lambda^{2}\right)^{1 / 4} \nabla g=\Phi_{1}(\lambda)\left(1+\lambda^{2}\right)^{1 / 2} g+\Phi_{2}(\lambda) g,
$$

and

$$
\mathcal{R}_{\mathcal{L}\left(L_{q}\left(B_{R}\right), L_{q}\left(B_{R}\right)^{N}\right)}\left(\left\{\left(\lambda \partial_{\lambda}\right)^{\ell} \Phi_{1}(\lambda) \mid \lambda \in \mathbb{R} \backslash\{0\}\right\}\right) \leq r_{b},
$$




$$
\mathcal{R}_{\mathcal{L}\left(H_{q}^{2}\left(B_{R}\right), L_{q}\left(B_{R}\right)^{N}\right)}\left(\left\{\left(\lambda \partial_{\lambda}\right)^{\ell} \Phi_{1}(\lambda) \mid \lambda \in \mathbb{R} \backslash\{0\}\right\}\right) \leq r_{b},
$$

for $\ell=0,1$ with some constant $r_{b}$. Thus, by Weis' operator-valued Fourier multiplier theorem, Theorem 8, and transference theorem, Theorem 9, we have (5.25), which completes the proof of Lemma 23.

By (5.1), (2.5), (5.7) and (5.17), we have

$$
\begin{gathered}
\left\|\partial_{t} V_{g}(\mathbf{k})\left(H_{h}, \nabla H_{h}\right)\right\|_{L_{\infty}\left((0,2 \pi), L_{q}\left(B_{R}\right)\right)} \leq C\|h\|_{H_{p}^{1}\left((0,2 \pi), W_{q}^{1-1 / q}\left(B_{R}\right)\right)} \leq C \epsilon, \\
\left\|V_{g}(\mathbf{k})\left(H_{h}, \nabla H_{h}\right)\right\|_{L_{\infty}\left((0,2 \pi), H_{q}^{1}\left(B_{R}\right)\right)} \leq C\left\|H_{h}\right\|_{L_{\infty}\left((0,2 \pi), H_{q}^{2}\left(B_{R}\right)\right)} \leq C \epsilon
\end{gathered}
$$

Thus, by Lemma 22, Lemma 23, and (5.1), we have

$$
\begin{aligned}
& \|g(\mathbf{v}, h)\|_{H_{p}^{1 / 2}\left((0,2 \pi), L_{q}\left(B_{R}\right)\right)}+\|g(\mathbf{v}, h)\|_{L_{p}\left((0,2 \pi), H_{q}^{1}\left(B_{R}\right)\right)} \\
& \quad \leq C \epsilon\left(\|\nabla \mathbf{v}\|_{H_{p}^{1 / 2}\left((0,2 \pi), L_{q}\left(B_{R}\right)\right)}+\|\nabla \mathbf{v}\|_{L_{p}\left((0,2 \pi), H_{q}^{1}\left(B_{R}\right)\right)}\right) \\
& \quad \leq C \epsilon\left(\|\mathbf{v}\|_{L_{p}\left((0,2 \pi), H_{q}^{2}\left(B_{R}\right)\right)}+\left\|\partial_{t} \mathbf{v}\right\|_{\left.L_{p}\left((0,2 \pi), L_{q}\left(B_{R}\right)\right)\right)}\right. \\
& \quad \leq C \epsilon^{2}
\end{aligned}
$$

Analogously, recalling the definition of $\mathbf{h}(\mathbf{v}, h)=\left(\mathbf{h}^{\prime}(\mathbf{v}, h), h_{N}(\mathbf{v}, h)\right)$ given in (3.31) and (3.34), where $\rho$ is replaced by $h$, and using Lemma 22, we have

$$
\begin{aligned}
& \|\mathbf{h}(\mathbf{v}, h)\|_{H_{p}^{1 / 2}\left((0,2 \pi), L_{q}\left(B_{R}\right)\right)}+\|\mathbf{h}(\mathbf{v}, h)\|_{L_{p}\left((0,2 \pi), H_{q}^{1}\left(B_{R}\right)\right)} \\
& \quad \leq C \epsilon\left(\|\nabla \mathbf{v}\|_{H_{p}^{1 / 2}\left((0,2 \pi), L_{q}\left(B_{R}\right)\right)}+\|\nabla \mathbf{v}\|_{L_{p}\left((0,2 \pi), H_{q}^{1}\left(B_{R}\right)\right)}\right. \\
& \left.\quad+\left\|\bar{\nabla}^{2} H_{h}\right\|_{H_{p}^{1 / 2}\left((0,2 \pi), L_{q}\left(B_{R}\right)\right)}+\left\|\bar{\nabla}^{2} H_{h}\right\|_{L_{p}\left((0,2 \pi), H_{q}^{1}\left(B_{R}\right)\right)}\right) .
\end{aligned}
$$

Since $H_{p}^{1 / 2}\left((0,2 \pi), L_{q}\left(B_{R}\right)\right) \supset H_{p}^{1}\left((0,2 \pi), L_{q}\left(B_{R}\right)\right)$, we have

$$
\left\|\bar{\nabla}^{2} H_{h}\right\|_{H_{p}^{1 / 2}\left((0,2 \pi), L_{q}\left(B_{R}\right)\right)} \leq C\left\|\bar{\nabla}^{2} H_{h}\right\|_{H_{p}^{1}\left((0,2 \pi), L_{q}\left(B_{R}\right)\right)},
$$

and so using Lemma 23, (2.5), and (5.1), we have

$$
\begin{aligned}
& \|\mathbf{h}(\mathbf{v}, h)\|_{H_{p}^{1 / 2}\left((0,2 \pi), L_{q}\left(B_{R}\right)\right)}+\|\mathbf{h}(\mathbf{v}, h)\|_{L_{p}\left((0,2 \pi), H_{q}^{1}\left(B_{R}\right)\right)} \\
& \quad \leq C \epsilon\left(\|\mathbf{v}\|_{H_{p}^{1}\left((0,2 \pi), L_{q}\left(B_{R}\right)\right)}+\|\mathbf{v}\|_{L_{p}\left((0,2 \pi), H_{q}^{2}\left(B_{R}\right)\right)}\right. \\
& \left.\quad+\left\|\partial_{t} H_{h}\right\|_{L_{p}\left((0,2 \pi), H_{q}^{2}\left(B_{R}\right)\right)}+\left\|H_{h}\right\|_{L_{p}\left((0,2 \pi), H_{q}^{3}\left(B_{R}\right)\right)}\right) \\
& \quad \leq C \epsilon^{2} .
\end{aligned}
$$

Combining (5.15), (5.19), (5.20), (5.26), and (5.27) gives (5.6). Applying Theorem 6 to equations (5.5) and using (5.6) and (5.16) gives that

$$
\begin{aligned}
& \|\mathbf{u}\|_{L_{p}\left((0,2 \pi), H_{q}^{2}\left(B_{R}\right)\right)}+\left\|\partial_{t} \mathbf{u}\right\|_{L_{p}\left((0,2 \pi), L_{q}\left(B_{R}\right)\right)} \\
& \quad+\|\rho\|_{L_{p}\left((0,2 \pi), W_{q}^{3-1 / q}\left(S_{R}\right)\right)}+\left\|\partial_{t} \rho\right\|_{L_{p}\left((0,2 \pi), W_{q}^{2-1 / q}\left(S_{R}\right)\right)} \\
& \quad \leq M_{1}\|\mathbf{f}\|_{L_{p}\left((0,2 \pi), L_{q}(D)\right)}+M_{2} \epsilon^{2}
\end{aligned}
$$


for some constants $M_{1}$ and $M_{2}$ independent of $\epsilon \in(0,1)$. Finally, we estimate $\left\|\partial_{t} \rho\right\|_{L_{\infty}\left((0,2 \pi), W_{q}^{1-1 / q}\left(S_{R}\right)\right)}$. From the third equation in equations (5.5), we have

$$
\left\|\partial_{t} \rho\right\|_{W_{q}^{1-1 / q}\left(S_{R}\right)} \leq\|\mathcal{M} \rho\|_{W_{q}^{1-1 / q}\left(S_{R}\right)}+\|\mathcal{A} \mathbf{u}\|_{W_{q}^{1-1 / q}\left(S_{R}\right)}+\|\tilde{d}(\mathbf{v}, h)\|_{W_{q}^{1-1 / q}\left(S_{R}\right)} .
$$

Therefore, by (5.1), (5.7), (5.8), (5.11), (5.12), and (5.13), we have

$$
\begin{aligned}
\left\|\partial_{t} \rho\right\|_{L_{\infty}\left((0,2 \pi), W_{q}^{1-1 / q}\left(S_{R}\right)\right)} \leq & C\left(\|\mathbf{u}\|_{L_{p}\left((0,2 \pi), H_{q}^{2}\left(B_{R}\right)\right)}+\left\|\partial_{t} \mathbf{u}\right\|_{L_{p}\left((0,2 \pi), L_{q}\left(B_{R}\right)\right)}\right. \\
& +\|\rho\|_{L_{p}\left((0,2 \pi), W_{q}^{3-1 / q}\left(S_{R}\right)\right)} \\
& \left.+\left\|\partial_{t} \rho\right\|_{L_{p}\left((0,2 \pi), W_{q}^{2-1 / q}\left(S_{R}\right)\right)}+\epsilon^{2}\right),
\end{aligned}
$$

which, combined with (5.28), leads to

$$
E(\mathbf{u}, \rho) \leq M_{1}^{\prime}\|\mathbf{f}\|_{L_{p}\left((0,2 \pi), L_{q}(D)\right)}+M_{2}^{\prime} \epsilon^{2}
$$

for some constants $M_{1}^{\prime}$ and $M_{2}^{\prime}$ independent of $\epsilon \in(0,1)$. We choose $\epsilon>0$ so small that $M_{2}^{\prime} \epsilon<1 / 2$ and we assume that $M_{1}^{\prime}\|\mathbf{f}\|_{L_{p}\left((0,2 \pi), L_{q}(D)\right)} \leq \epsilon / 2$. Then, we have

$$
E(\mathbf{u}, \rho) \leq \epsilon .
$$

Moreover, by (2.5) and (5.8), we have

$$
\sup _{t \in(0,2 \pi)}\left\|H_{\rho}\right\|_{\left.H_{\infty}^{1}\left(B_{R}\right)\right)} \leq C\|\rho\|_{L_{\infty}\left((0,2 \pi), W_{q}^{1-1 / q}\left(S_{R}\right)\right)} \leq M_{3} E(\mathbf{u}, \rho) \leq M_{3} \epsilon .
$$

Choosing $\epsilon>0$ smaller if necessary, we may assume that $0<M_{3} \epsilon<\delta$, and so $(\mathbf{u}, \rho) \in \mathcal{I}_{\epsilon}$. If we define a map $\Phi$ acting on $(\mathbf{v}, h) \in \mathcal{I}_{\epsilon}$ by setting $\Phi(\mathbf{v}, h)=(\mathbf{u}, \rho)$, and then $\Phi$ is a map from $\mathcal{I}_{\epsilon}$ into itself. Employing a similar argument as for proving (5.30), we see that for any $\left(\mathbf{v}_{i}, h_{i}\right) \in \mathcal{I}_{\epsilon}(i=1,2)$,

$$
E\left(\Phi\left(v_{1}, h_{1}\right)-\Phi\left(v_{2}, h_{2}\right)\right) \leq M_{4} \in E\left(\left(\mathbf{v}_{1}, h_{1}\right)-\left(\mathbf{v}_{2}, h_{2}\right)\right) .
$$

Choosing $\epsilon>0$ smaller if necessary, we may assume that $M_{4} \epsilon \leq 1 / 2$, and so $\Phi$ is a contraction map on $\mathcal{I}_{\epsilon}$. The Banach fixed-point theorem yields the unique existence of a fixed point $(\mathbf{v}, \rho) \in \mathcal{I}_{\epsilon}$ of the map $\Phi$, that is $(\mathbf{v}, \rho)=\Phi(\mathbf{v}, \rho)$, which is the required solution of equations (2.16). This completes the proof of Theorem 4.

Open Access. This article is licensed under a Creative Commons Attribution 4.0 International License, which permits use, sharing, adaptation, distribution and reproduction in any medium or format, as long as you give appropriate credit to the original author(s) and the source, provide a link to the Creative Commons licence, and indicate if changes were made. The images or other third party material in this article are included in the article's Creative Commons licence, unless indicated otherwise in a credit line to the material. If material is not included in the article's Creative Commons licence and your intended use is not permitted by statutory regulation or exceeds the permitted use, you will need to obtain permission directly from the copyright holder. To view a copy of this licence, visit http://creativecommons.org/licenses/ by $/ 4.0 /$.

Publisher's Note Springer Nature remains neutral with regard to jurisdictional claims in published maps and institutional affiliations. 


\section{REFERENCES}

[1] K. de Leeuw, On L $L_{p}$ multipliers, Ann. Math., 81(2) (1965), 364-379.

[2] T. Eiter, M. Kyed, and Y. Shibata, $\mathcal{R}$-solvers and their application to periodic $L_{p}$ estimates, Preprint in 2019.

[3] G. P. Galdi, M. Kyed, Time-periodic solutions to the Navier-Stokes equations, Handbook of mathematical analysis in mechanics of viscous fluids, 509-578, Springer, Cham, 2018.

[4] G. P. Galdi, H. Sohr, Existence and uniqueness of time-periodic physically reasonable viscous fluid flow problems, Arch. Rational Mech. Anal., 172 (2004), 363-406.

[5] M. Geissert, M. Hieber, T. H. Nguyen, A general approach to time periodic incompressible viscous fluid flow problems, Arch. Rational Mech. Anal., 220 (2016), 1095-1118.

[6] M. Hieber, A. Mahalov, R. Takada, Time periodic and almost time periodic solutions to rotating stratified fluids subject to large forces, J. Differential Equations 266 (2019), 977-1002.

[7] M. Hieber, T. H. Nguyen, A. Seyfert, On periodic and almost periodic solutions to incompressible viscous fluid flow problems on the whole line, Mathematics for nonlinear phenomena-analysis and computation, 51-81, Springer Proc. Math. Stat., 215, Springer, Cham, 2017.

[8] H. Kozono, M. Nakao, Periodic solutions of the Navier-Stokes equations in unbounded domains, Tohoku Math. J. 48(2) (1996), 33-50.

[9] A. Lunardi, Interpolation Theory, 16 Appunti Lecture Notes, Edizioni della Normale, Scuola Normale Superiore Pisa, 2018, https://doi.org/10.1007/978-88-7642-638-4

[10] P. Maremonti, Existence and stability of time-periodic solutions to the Navier-Stokes equations in the whole space, Nonlinearity 4 (1991), 503-529.

[11] P. Maremonti, M. Padula, Existence, uniqueness and attainability of periodic solutions of the navierStokes equations in exterior domains, Zap. Nauchn. Sem. S.-Peterburg. Otdel. Mat. Inst. Steklov (POMI) 233 (1996), Kraev. Zadachi Mat. Fiz. i Smezh. Vopr. Teor. Funkts., 27, 142-182, 257; translation in J. Math. Sci. (new York) 93 (1999), 719-746.

[12] T. Okabe, Y. Tsutsumi, Time periodic strong solutions to the incompressibel Navier-Stokes equations with external forces of non-divergence form, J. Differential Equations 263 (2017), 8229-8263.

[13] R. Salvi, On the existence of periodic weak solutions on the Navier-Stokes equations in exterior regions with periodically moving boundaries, Navier-Stokes equations and related nonlinear problems (Funchal, 1994), 63-73, Plenum, New York, 1995.

[14] J. Serrin, A note on the existence of periodic solutions of the Navier-Stokes equations, Arch. Rational Mech. Anal., 3 (1959), 120-122.

[15] Y. Shibata, On the $\mathcal{R}$-boundedness of solution operators for the Stokes equations with free boundary condition, Diff. Int. Eqns. 27(3-4) (2014), 313-368.

[16] Y. Shibata, On the $\mathcal{R}$-bounded solution operators in the study of free boundary problem for the Navier-Stokes equations, Springer Proceedings in Mathematics \& Statistics Vol. 183 2016, Mathematical Fluid Dynamics, Present and Future, Tokyo, Japan, November 2014, ed. Y. Shibata and Y. Suzuki, pp.203-285.

[17] Y. Shibata, On the local wellposedness of free boundary problem for the Navier-Stokes equations in an exterior domain, Comm. Pure, Appl. Anal., 17 (4) (2018), 1681-1721, https://doi.org/10.3934/ cpaa.2018081.

[18] Y. Shibata, $\mathcal{R}$-boundedness, maximal regularity and free boundary problems for the Navier Stokes equations, Mathematical Analysis of the Navier-Stokes Equations: Cetraro, Italy 2017. G. P. Galdi and Y. Shibata (eds), C.I.M.E. Foundation in Subseries Book 2254, Springer 2020, pp. 193-462. ISBN 978-3-030-36226-3

[19] Y. Shibata and H. Saito, Global Well-posedness for Incompressible-Incompressible Two Phase Problem, In: T. Bodnar, G.P. Galdi, S. Necasova (eitors): Fluids under Pressure, Book Series: Advances in Mathematical Fluid Mechanics, Birkhauser (2020)

[20] Y. Taniuchi, On stability of periodic solutions of the Navier-Stokes equations in unbounded domains, Hokkaido Math. J., 28 (1999), 147-173.

[21] H. Triebel, Interpolation Theory, Function Spaces, Differential Operators, North-Holland, Amsterdam (1978).

[22] L. Weis, Operator-valued Fourier multiplier theorems and maximal $L_{p}$-regularity. Math. Ann. 319 (2001), 735-758. 
[23] M. Yamazaki, The Navier-Stokes equations in the weak- $L^{n}$ space with time-dependent external force, Math. Ann., 317 (2000), 635-675.

\author{
Thomas Eiter \\ Weierstrass Institute for Applied \\ Analysis and Stochastics \\ Mohrenstr. 39 \\ 10117 Berlin \\ Germany \\ E-mail:thomas.eiter@wias-berlin.de \\ Mads Kyed \\ Hochschule Flensburg \\ Kanzleistraße 91-93 \\ 24943 Flensburg \\ Germany \\ E-mail: mads.kyed@hs-flensburg.de \\ Yoshihiro Shibata \\ Department of Mathematics \\ Waseda University \\ Ohkubo 3-4-1, Shinjuku-ku \\ Tokyo 169-8555 \\ Japan \\ E-mail: yshibata325@gmail.com
}

Accepted: 7 September 2020 